Portland State University

PDXScholar

$1-1-2011$

\title{
A Computational Model of Adaptive Sensory Processing in the Electroreception of Mormyrid Electric Fish
}

Eran Agmon

Portland State University

Follow this and additional works at: https://pdxscholar.library.pdx.edu/open_access_etds Let us know how access to this document benefits you.

Recommended Citation

Agmon, Eran, "A Computational Model of Adaptive Sensory Processing in the Electroreception of Mormyrid Electric Fish" (2011). Dissertations and Theses. Paper 291.

https://doi.org/10.15760/etd.291

This Thesis is brought to you for free and open access. It has been accepted for inclusion in Dissertations and Theses by an authorized administrator of PDXScholar. Please contact us if we can make this document more accessible: pdxscholar@pdx.edu. 


\section{A Computational Model of}

Adaptive Sensory Processing in the Electroreception of Mormyrid Electric Fish

by

Eran Agmon

A thesis submitted in partial fulfillment of the requirements for the degree of

Master of Science

in

Systems Science

Thesis Committee:

George G. Lendaris, Chair

Patrick D. Roberts

Randy Zelick

Portland State University

C)2011 


\section{Abstract}

Electroreception is a sensory modality found in some fish that enables them to sense the environment through the detection of electric fields. Biological experimentation on this ability has built an intricate framework that has identified many of the components involved in electroreception's production, but lack the framework for bringing the details back together into a system-level model of how they operate together. This thesis creates and tests a computational model of the Electrosensory Lateral Line Lobe (ELL) in mormyrid electric fish in an attempt to bring some of electroreception's structural details together to help explain its function. The ELL is a brain region that functions as a primary processing area of electroreception. It acts as an adaptive filter that learns to predict reoccurring stimuli and removes them from its sensory stream, passing only novel inputs to other brain regions for further processing. By creating a model of the ELL, the relevant components which underlie the ELL's functional, electrophysiological patterns can be identified and scientific hypotheses regarding their behavior can be tested.

Systems science's approach is adopted to identify the ELL's relevant components and bring them together into a unified conceptual framework. The methodological framework of computational neuroscience is used to create a computational model of this structure of relevant components and to simulate their interactions. Individual activation tendencies of the different included cell types are modeled with dynamical systems equations and are interconnected according to the connectivity of the real ELL. Several of the ELL's input patterns are modeled and incorporated in the model. The 
computational approach claims that if all of the relevant components of a system are captured and interconnected accurately in a computer program, then when provided with accurate representations of the inputs a simulation should produce functional patterns similar to those of the real system. These simulated patterns generated by the ELL model are compared to recordings from real mormyrid ELLs and their correspondences validate or nullify the model's integrity.

By building a computation model that can capture the relevant components of the ELL's structure and through simulation reproduces its function, a systems-level understanding begins to emerge and leads to a description of how the ELL's structure, along with relevant inputs, generate its function. The model can be manipulated more easily than a biological ELL, and allows us to test hypotheses regarding how changes in the structures affect the function, and how different inputs propagate through the structure in a way that produces complex functional patterns. 


\section{Contents}

$\begin{array}{ll}\text { Abstract } & \text { i }\end{array}$

Contents

List of Figures $\quad$ V

1 Introduction 1

1.1 Overview and organization

1.2 Summary of contributions

2 Background

2.1 The evolution of electroreception

2.2 Electrosensory behavioral function

2.3 The structure of electroreception

2.4 The electrosensory lateral line lobe

2.4.1 Synaptic plasticity

2.5 Summary

3 Formulation of Research Topic

3.1 The ELL's relevant components

3.1.1 Across the brain, body, and environment

3.2 The function of a model

3.3 Summary

4 Description of Approach

4.1 Computational neuroscience

4.1.1 Two-dimensional integrate-and-fire neurons

4.2 Computational model of electroreception

4.3 Simulation

4.4 Summary

5 Methodology

5.1 The Roberts-Kusefoglu model

5.2 Additions

5.2.1 Corollary discharge

5.2.2 Synaptic plasticity

5.2.3 Broad spikes

5.2.4 Proprioception

5.2.5 Body motion effects

5.2.6 Efferent cells 
5.3 Simulation and visualization

6 Result

6.1 MG cell activity

6.2 Efferent cell activity

6.3 Removing the sensory consequences of tail movement

6.4 Testing hypotheses

6.4 Discussion

7 Concluding Remarks

7.1 Contributions

7.2 Limitations

7.3 Future work

7.4 Conclusion

References 


\section{List of Figures}

\subsection{Elephant Nose Fish}

2.2 Distortion of Electric Field by Nearby Objects (From von der Emde, 1999)

2.3 Spike latency is inversely proportional to stimulation intensity. (Han, Grant, \& Bell 2000)

2.4 Extrinsic circuitry of the medial zone of the ELL (Meek et al. 1999)

2.5 Intrinsic circuitry of the medial zone of the ELL (Meek et al. 1999)

2.6 Hypothesized functional circuit of the ELL (From Han 1999)

2.7 Major Features of a Cerebellum-like Structure (Bell, 2001)

2.8 Anti-Hebbian Plasticity displayed in MG cells (Bell, 1997)

3.1 Tail position's effect on cellular activity (Sawtell \& Williams 2008, Sawtell 2010)

3.2 Afferent and efferent informational measures (Sawtell \& Williams, 2008)

4.1 Hodgkin-Huxley components (Hodgkin \& Huxley 1952)

4.2 Variety of possible patterns generated by a 2-d IF model (Izhikevich 2007)

4.3 Anti-Hebbian STDP in MG cells (Bell, 1997)

5.1 The Roberts-Kusefoglu model (Roberts 2010)

5.2 Hypothesized functional circuit of the ELL (Han 1999)

5.3 Granule Cell Activity resulting from the 2d-IF parameters of Table I

5.4 Afferent Cell Activity resulting from the 2d-IF parameters of Table II

5.5 MG Cell Activity resulting from the 2d-IF parameters of Table III

5.6 Cells' spike times in response to Gaussian input (Roberts 2010)

5.7 A single parallel fiber input

5.8 Total current, consisting of added up parallel fiber inputs from EOCD

5.9 Model of synaptic plasticity

5.10 Weights after synaptic plasticity

5.11 EOCD input after weights are multiplied

5.12 Proprioceptive input

5.13 Efferent cell activity (Mohr, Roberts, Bell 2003)

5.14 Efferent cell simulation with the 2d-IF parameters of Table IV

6.1 MG1 raster plot, simulation

6.2 MG2 raster plot, simulation

6.3 MG1 raster plot, electrophysiological data (Sawtell 2007)

6.4 MG2 raster plot, electrophysiological data (Mohr 2003)

6.5 Simulated efferent activity

6.6 Efferent activity (Mohr 2003)

6.7 Tail position's effect on cellular activity (Sawtell \& Williams 2008, Sawtell 2010)

6.8 MG1 (left) \& MG2 (right) pre-training

6.9 MG1 (left) \& MG2 (right) post-training

6.10 Efferents pre-training

6.11 Efferents post-training 


\section{Chapter 1}

\section{Introduction}

This introductory chapter provides an overview of the motivation and central argument advanced in this thesis. It includes details about how the thesis is organized, and discusses the thesis's original contributions.

\subsection{Overview and organization}

The underlying motivation for this thesis is to bridge the gap between the scientific understanding of the neural structure of the Electrosensory Lateral Line Lobe (ELL) in Mormyrid Electric Fish, and its function of adaptive sensory processing. This is done by building a computational model of the ELL and then simulating it in different contexts to test its ability to predict patterns seen in real ELLs. A successful model, which reproduces the ELL's neurophysiological patterns, can provide great insight into the working of the ELL by pointing to the underlying mechanisms of the adaptive filter function. Such a model can also be used to test hypotheses regarding the ELL more easily than through biological experimentation.

Scientific work in the field of biology has built thorough descriptions of the ELL's structure and function. This biological background is discussed in Chapter 2, and is used to build up the current scientific understanding of the ELL. By providing the background, the reader is brought up to date to the underlying scientific problems faced by this thesis. These problems can be generally stated to be the dichotomy between the structure and the function of the ELL as a system. The components of the ELL have been 
studied extensively, but how they interact together and with the environment to produce the ELL's function is not understood. The thesis aims to consolidate the scientific knowledge regarding the ELL's structural neuroanatomy and its functional, physiological patterns of activity.

The prevailing hypothesis regarding the function of the ELL, which is adopted in this thesis, is that the ELL serves as an adaptive filter of electrosensory data. In short, the adaptive filter hypothesis claims that by integrating the data received from its sources through its neural structure, the ELL filters predictable data out of the sensory stream. This is demonstrated by its efferents, which carry novel data that can predict important aspects of the nearby environment. The filter adapts through experience and learns to predict stimuli by creating a "negative image" of predictable stimuli, which is combined with the incoming sensory stream in a way that removes the predictable signals and allows only for novel features to pass. This adaptive filter function hypothesis is discussed in greater detail in Chapter 2.

This thesis emphasizes systems science's conceptual framework combined with the computational approach as a viable way to understand how the ELL's structure is able to produce the adaptive filter function. The conceptual framework of systems science, which is discussed in Chapter 3, describes a system's structure as a network of components, which operate together in a way that produces the system's behavior as a whole. The function can be regarded as the system's relation to other systems in its environment, defined by its inputs and outputs. The ELL is examined with a systems science perspective in search of the relevant components underlying the adaptive filter function. Once this systems framework of the ELL is in place, Chapter 4 discusses the 
computational approach and how it can create a model of the ELL's organization based on this systems framework. A successful model of the ELL would provide a link between structure and function, and would allow scientists to test hypotheses regarding the ELL by simulating it in a computer program.

The specific methodology required for the computational modeling of the ELL is discussed in Chapter 5. The model created for this thesis builds upon a previous model of the ELL, which was created by Patrick Roberts and Deniz Kusefoglu in 2009. This previous model uses the dynamical systems approach for the capturing of the quantitative behavior of neuronal activity within the ELL. Differential equations are tuned to capture the specific behavior of individual cell types, and can predict their behavior in responses to input patterns. By connecting the different represented cells' inputs and outputs; a network structure is constructed that mirrors that of the ELL. The differential equations are able to generate the activity of the different cells within the network when configured correctly and provided with input.

Additions made to this previous model allow the adaptive filter function to be examined. These additions include an additional cell type, a more accurate and complete set of inputs, and a learning rule that captures the cells' synaptic plasticity. By incorporating these components, the ELL's adaptive filter function can studied more extensively because the additions would allow for synaptic plasticity to work on the cells in a way that is accurate to the real ELLs. Synaptic plasticity is the basis for change and adaptation that makes the adaptive filter possible.

After describing how this model was built, its simulations result are visualized and discussed in Chapter 6. The model is simulated through various scenarios, and the 
recorded results were compared to electrophysiological patterns observed in real ELL's. If our scientific understanding of the ELL's structure is good enough to captures the relevant elements, and the computational model accurately captures these details, then it should reproduce the patterns of an adaptive filter function. If the simulation generates these patterns, then the scientific model is verified. By looking at visualizations of data obtain from simulation and comparing it with to data from real ELL's, the model's success is assessed as well as its limitations and failures. A successful model can be used to test hypotheses and pose predictions about real ELLs. Some such hypotheses regarding the ELL are tested and their results discussed.

Chapter 7 is a conclusion, which brings the results back into a broader perspective regarding the role of the ELL and the newly-formed bridge between our scientific knowledge of the ELL's structure and function. The main contribution provided by this thesis is a working computational model of the main components underlying the ELL's function, which can be extended and tested in future studies. Simulation results demonstrate the sufficiency of the incorporated components in production of the ELL's electrophysiological patterns, and provide insight to multiple hypotheses currently explored in the field. The limitations of the computational model are described, and then future additions that can extend the model's descriptive ability are speculated upon. 


\section{Chapter 2}

\section{Background}

This chapter provides an account of previous scientific explorations of mormyrid electroreception. By looking through the field's history, we can work towards the present issues faced in this thesis. The biological sciences, which include neuroscience, have been the major drivers of previous research on the subject of electroreception. By decomposing electroreception, biologists have been able to identify many of the major contributing components. These components are arranged across multiple levels of phenomena across the boundaries of the environment, the body, and the brain. The biological framework has studied the different components of each of these levels, and provided rich descriptions of them.

Electroreception can be scrutinized from multiple perspectives. Section 2.1 discusses the evolutionary perspective on electroreception, which looks to the evolution of electrosensation and the various forms of electrosensation that have evolved in the different electrical fishes of the world. Section 2.2 looks at the perspective of electrosensation as a behavior within an environment. This sees the organism as a component within a broader suprasystem, in which the organism interacts with the other systems of the environment. In this level electroreception is studied through the electrical inputs and outputs produced by the organism. Section 2.3 looks at electroreception through the structure required to generate it in an organism. The structure includes the body of the fish, whose movement is coordinated through a subsystem: the nervous 
system. Section 2.4 describes the electrosensory lateral line lobe (ELL), a brain region that performs primary sensory processing of electrosensory data.

\subsection{The evolution of electroreception}

The electric fish of interest to this thesis is the mormyrid species Gnathonemus petersii. This species is known as elephant nose fish because of a trunk-like lower lip that evolved to help them feed upon small invertebrates that lay buried in the mud (see Fig. 2.1). Mormyrids have the behavior known as active electrosensation, which arises through the coupling of electroreception and electrogeneration. Electroreception happens in sensory organs that activate in response to electric charge, they are called electroreceptors. Electrogeneration is the generation of an electric field in surrounding waters. Electric organs discharge into the water to produce an electric organ discharge (EOD). By pairing the generation of a field with the sensation of a field, mormyrids can detect their own generated field. They use time signals regarding the generation of the field along with sensed electrical activity in the water to deduce information regarding their environment. Mormyrids are just one family electric fish native to Africa. There are numerous other African fish with electrosensation, as well as a many fish in South America.

Electrosensation appears to have evolved independently at least four times in evolutionary history. Electroreceptors are present in most non-teleost fish and in four orders of teleosts. The non-teleost fish are with electroreceptors are Agnatha, Elasmobranchii, Holocephali, Chondrostei, Polypteri, and Dipnoi. The four orders of teleost fish are the siluriforms (catfishes), the gymnotiforms (knifefishes), the 
mormyriforms (elephant nose fishes), and in one subfamily (Xenomystinae) of the osteoglosiforms. (Zupanc and Bullock 2005).

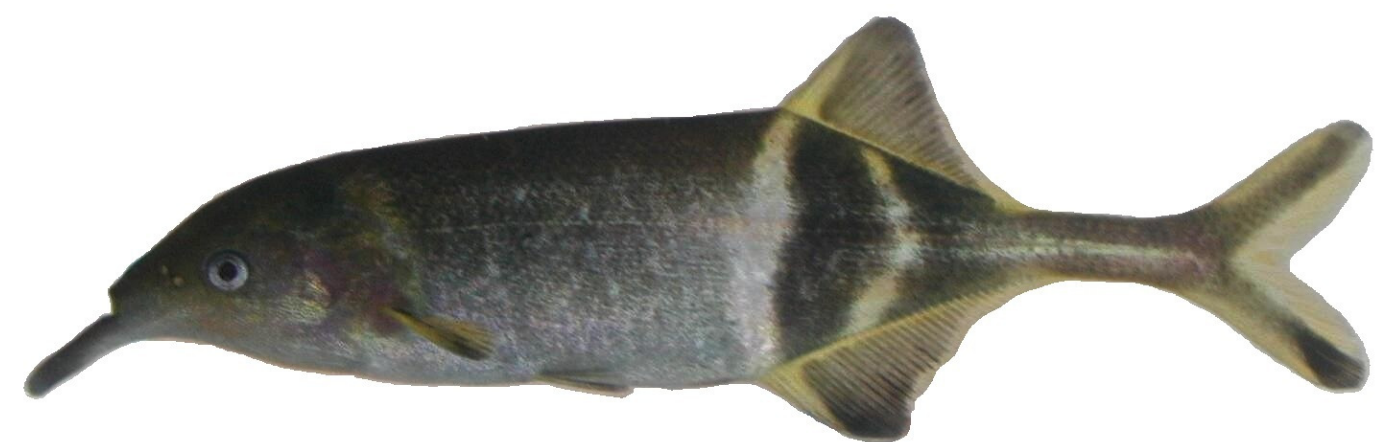

Fig. 2.1: Elephant Nose Fish (Gnathonemus petersii)

Elephant Nose Fish are a species of mormyrid fish, native to Africa. Their name comes from their trunk-like lower lip that helps them feed on prey that lays beneath the mud. http://www.bio.davidson.edu/people/midorcas/animalphysiology/websites/2003/Wilson/ GalONE.htm

Meanwhile, electric organs are believed to have evolved at least six times independently among fishes. They evolved later than electroreceptors, perhaps to complement the receptors by creating EODs that can be detected by the electroreceptors. Two types of EODs have been observed in different electric fish: pulse-like EODs and wave -like EODs. Almost all mormyrids have pulse-like EODs, which consist of brief, stereotyped pulses which are separated by intervals of no activity. Other species have wave-like discharges, which produce stable fluctuations of electrical amplitude across long periods of time (Zupanc and Bullock 2005).

Controlled electric currents are generated by an electric organ, and propagate through the environment, and leads to electrical interaction with external systems such as rocks, foliage, and other organisms. The interaction with external systems distorts the field's shape, and these distortions can be detected by the electroreceptors. The mormyrid brain retains information from the brain region that initiated the electric pulse 
in a signal called the "electric organ corollary discharge' (EOCD). This signal is combined with the information regarding sensed electrical signal in the environment in a way that environmental context is deduced. This is a beneficial ability that allows fish to detect their environment in conditions when vision is limiting. Evolutionary history points to the success of electroreception as a biological trait due to the large amount of species that have it and their ability to succeed in their environment.

\subsection{Electrosensory behavioral function}

Active electrosensation is a behavior that results from the combination of being electrogenerative and electroreceptive. It gives mormyrids and other electric fish the interesting ability of being able create an electric field and then detect it. Mormyrids have evolved to take advantage of this ability, and use it to perceive objects within their environment. Objects in the nearby environment distort the generated electric field due to their different electric conductances. These distortions change the electrical image that along the mormyrids' body, and therefore the activation pattern of their electroreceptors. Mormyrids have neural circuitry that can process the activation patterns of their electroreceptors, in combination with the pattern of electric generation, to localize nearby objects and identify some of their electrical properties. Through sequences of electrogeneration and electroreception, mormyrids can reliably perceive their environment, which allows it to interact with the world in a way that allows it to accomplish basic goals, such as approaching and catching prey.

Mormyrid electroreception can be split into two main functions: passive sensation and active sensation. Passive sensation is the detection of bioelectric fields generated by 
other animals in the mormyrids' proximity. This makes use of certain type of electroreceptor call ampullary receptors, which detect low-frequency electric fields. These are discussed in greater detail in the next section on the structure of electroreception. Active sensation is the activation of electroreceptors in response to the electric field generated by the EOD. This type of reception is done primarily by tuberous receptors because they are better tuned to the frequencies of electric activity generated by the EOD. Of the two type of tuberous receptors, mormyromasts specialize in the detection of EODs generated by the fish and knollenorgans specialize in detection of EODs generated by other fish of the same species.

Objects in surrounding water usually have different electric conductances, which allow electric currents to pass through them with different levels of ease. Objects with conductance values lower than the surrounding water attract electric current; objects with conductance values higher than surrounding waters repel electric current. If they are within a certain distance the mormyrid (depending on the fish's size), these different conductances interact with and distort the generated electric field. The change in the electric field due to the presence of an object lead to distortions of the electric field along the mormyrids' bodies, called the "electric image" of the objects. This is diagrammed in Fig. 2.2. Electroreceptors are activated according to this distortion, and their activation information is passed to neuronal processing areas that can deduce properties of the external object such as relative location, size, shape, and electrical properties such as resistive and capacitive components (von der Emde, 2006). 


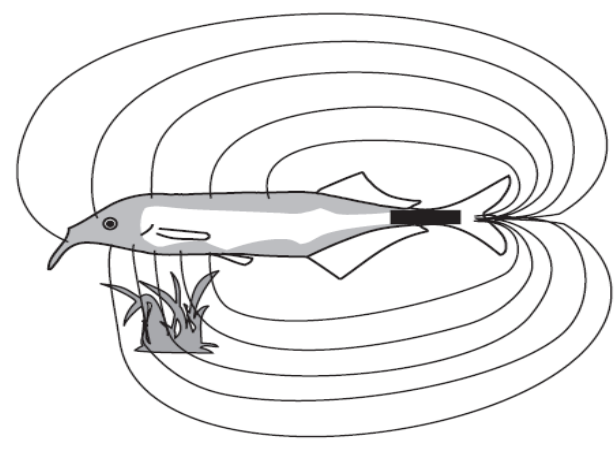

Conductor

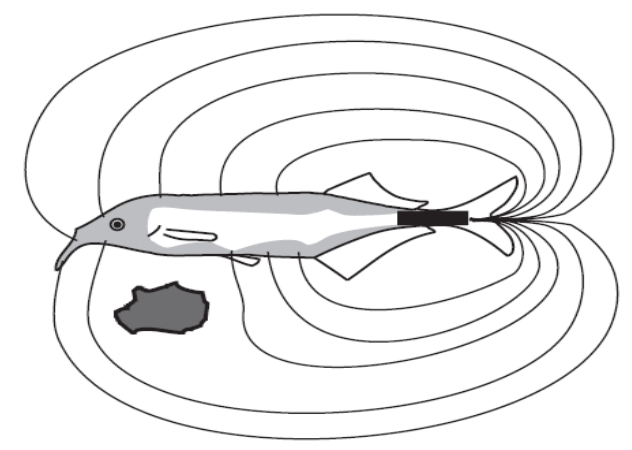

Non-conductor

Fig. 2.2: Distortion of Electric Field by Nearby Objects (von der Emde, 1999)

Mormyrids have also further evolved their electrosensory ability in a way that allows for communication through the detection of other electric fish's EODs. Specialized electroreceptors called knollenorgans, which will be discussed in the next section, evolved to detect these different EODs. This ability allows for species recognition (Zupanc and Bullock 2005), intraspecific communication (Hopkins), detection of gender differences (Harold Zakon), and individual recognition and coordination (Arnegard 2006). Species recognition has evolved by developing a wide repertoire of variants of pulse-likes EODs. Electric fish can detect these other EODs generated by different species for recognizing and communicating with the species. Individual differences within a species allows for recognition. This ability is used for courting, through gender-specific signals. It also allows for the detection of individual differences and used for pack hunting, as observed in mormyrids.

\subsection{The structure of electroreception}

The previous section described the functional, behavioral perspective on electroreception. But one could also take a structural perspective, which looks at electroreception through the interactions of biological components within the mormyrid fish. The mormyrid is an 
organism, composed of body parts that are interconnected and operate together to produce behavior within the environment. The electric discharge is generated by an electric organ in the stem of their tail that evolved from a muscle. Electroreceptors are embedded in the organism's skin, with certain body regions more densely packed with receptors than others. Muscles and bones move the fish's body into different positions that allow it to maneuver within its environment. As the body moves, so does the electric organ and electroreceptors. This create changes in how the electric field is distributed around the fishes' body, and therefore in activation pattern of the fishes' electroreceptors. The nervous system processes these patterns of inputs received by the electroreceptors, and it controls the outputs of body movement and electric organ discharge. It is through the structure of the body and brain that the mormyrid is able to coordinate electroreception.

The electric organ is able to produce its discharge (EOD) through electrically excitable cells called 'electrocytes.' When these cells receive simultaneous command from the brain to fire, they pass an electric current along the axis of the organ and into the surrounding water. (Hopkins, 1974). The physics of the organ and the EOD have been extensively studied and modeled. The number of electrocytes arranged in series determines the voltage; the number of electrocytes arranged in parallel determines the current (Zupanc \& Bullock, 2005). This organ is interfaced by neurons that carry signals from the electromotor command nucleus in the brainstem, which controls the timing of EOD. (Bell 1999).

Electrosensation originates in electroreceptors, which are sensory receptors that detect electric charge. Such receptors are embedded in the fishes' skin, just like tactile 
receptors are embedded in our own skin. There are two classes of electroreceptors that are found in mormyrids: ampullary and tuberous. Ampullary organs are characterized by a tube-like structure that starts with a pore in the skin and ends with a sensory epithelium. They are sensitive to DC and low-frequency AC electric fields, which lets them detect the bioelectric discharge of other animals. Tuberous organs are different from ampullary organs in that they are not connected to the surface of the skin, but are covered by loosely packed epidermal cells. (Jørgensen, 2005) The tuberous organs of mormyrids are divided into two main types: knollenorgans and mormyromasts (Zakon, 1986). Knollenorgans are highly sensitive but do not encode the stimulus' strength. Mormyromasts are sensitive to the amplitude of the electric field, and are generally tuned to detect frequencies near the specific frequency of the mormyrid's EOD. This makes the mormyromasts sensitive to the electric field generated by the electric organ. The latency of the spikes in mormyromast afferents reflects the intensity of the electric field at the receptor. Weak fields generate a single spike with a latency from the EOD of up to 12 milliseconds, and strong fields generate multiple spikes with a latency of at least two milliseconds (Bell, 1990). This principle is demonstrated in Fig. 2.3.

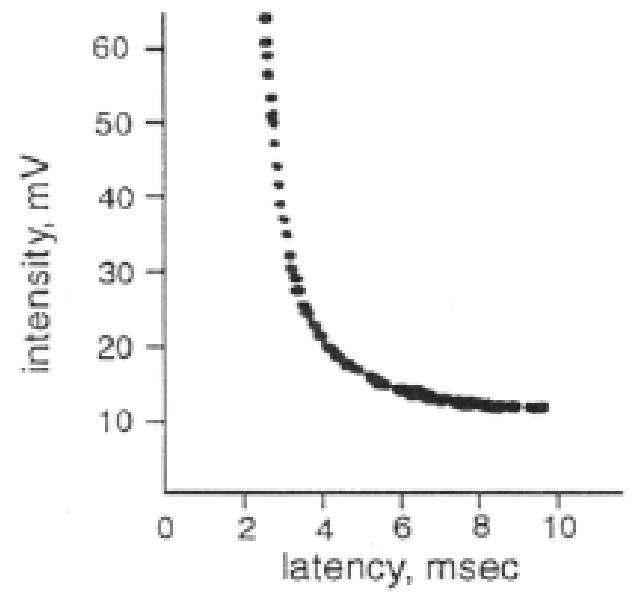

Fig. 2.3: Spike latency is inversely proportional to stimulation intensity. (Han, Grant, \& Bell 2000) 


\section{4 Electrosensory lateral line lobe}

The distorted electric field, which is sensed by the electroreceptors, is passed on to the brain for further processing and the determining of environmental context. As mentioned in the introduction, the primary processing region for electrosensory data is the ELL. The ELL is found on the roof of the mormyrids' rhombencephalon. The extrinsic circuitry of the ELL with other brain regions is diagrammed in Fig 2.4. This figure shows the three main inputs that the ELL receives from other brain regions. The first input is the electrosensory inputs (shown as the green connection coming from the electroreceptors labeled $a o, k o$, and morm). It receives this direct input from electroreceptor afferents in a topographic manner that retains the relative spatial locations of the sensory inputs. A second source of input is the corollary electromotor command (EOCD) signals; the connections are shown in green from the region labeled "n1". The EOCD originates in juxtalobar nucleus (JLN), which triggers the electric organ discharge. The signal it carries temporal information about the discharge to the ELL. The third major set of inputs comes from proprioceptive brain areas that regarding the animal's own body position (Sawtell \& Williams 2008). These are shown in purple in Fig. 2.4.

The electroreceptor afferents provide the ELL with information about the spatial distribution of electric current and the timing of receptor activation. Proprioceptive input provides information regarding body position, which is critical for interpreting the electrosensory input that was shaped by the body's position. The EOCD signal is timelocked with the EOD motor command, and provides the ELL with information about the timing of EOD. It has been shown that the ELL integrates signals from these sources, and acts as an adaptive filter to remove predictable input from the sensory stream. The 
ELL contains a cerebellar-like structure with GABAergic Purkinje-like medium ganglion (MG) cells, which display strong synaptic plasticity. This particular structure has been shown to take noisy, external signals and pull out the novel stimuli. It is hypothesized to occur due to the MG cells learning the predictable stimuli and reducing the strength of its signal through anti-Hebbian plasticity. By reducing the influence out predicable data, the ELL's output contains only novel stimuli, and is able to predict information about the basic properties of nearby objects that are novel, such as their location and electrical conductivity (Sawtell 2008). This is a critical piece of the information processing that allows the mormyrid to sense the environment and make informed decisions about how to behave within this environment.

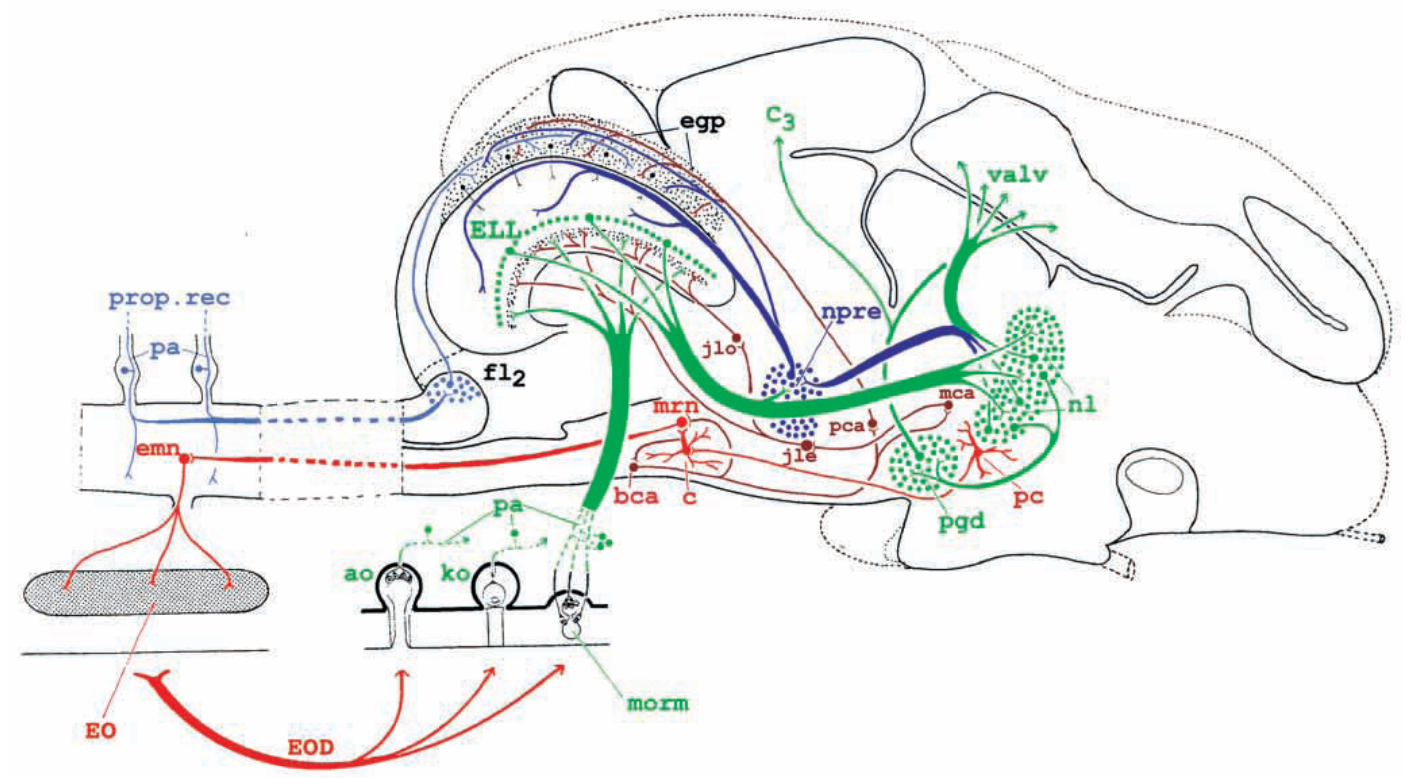

Fig. 2.4: Extrinsic circuitry of the medial zone of the ELL (Meek et al. 1999)

Electrosensory inputs come to the ELL through the projections indicated in green that are connected to the receptors labeled "morm" for mormyromast, "ko" for knollenorgans, and "ao" for ampullary organs. Corollary discharge projections are also indicated in green from the area labeled "n1." This same region has projections to the electric organ, which are colored red. Proprioceptive inputs are colored purple. 
The ELL's structure is organized in a complex manner. It is made of many cell types and contains many connections. The structure of interest to this thesis is the cerebellum-like structure that is embedded within the ELL. This structure is defined by the laminar organization around a layer of Purkinje-like MG cells. The complex intrinsic anatomy of the ELL that has been mapped is diagrammed in Fig. 2.5. Within this structure exists a reduced cerebellum-like structure, diagrammed in Fig. 2.6. This cerebellum-like structure is adopted in this thesis as the relevant structure for the adaptive filter function. The many other cell types and connections within the ELL that are disregarded in this model are hypothesized to serve different functions other than the adaptive filter, and might be used for different types of processing.

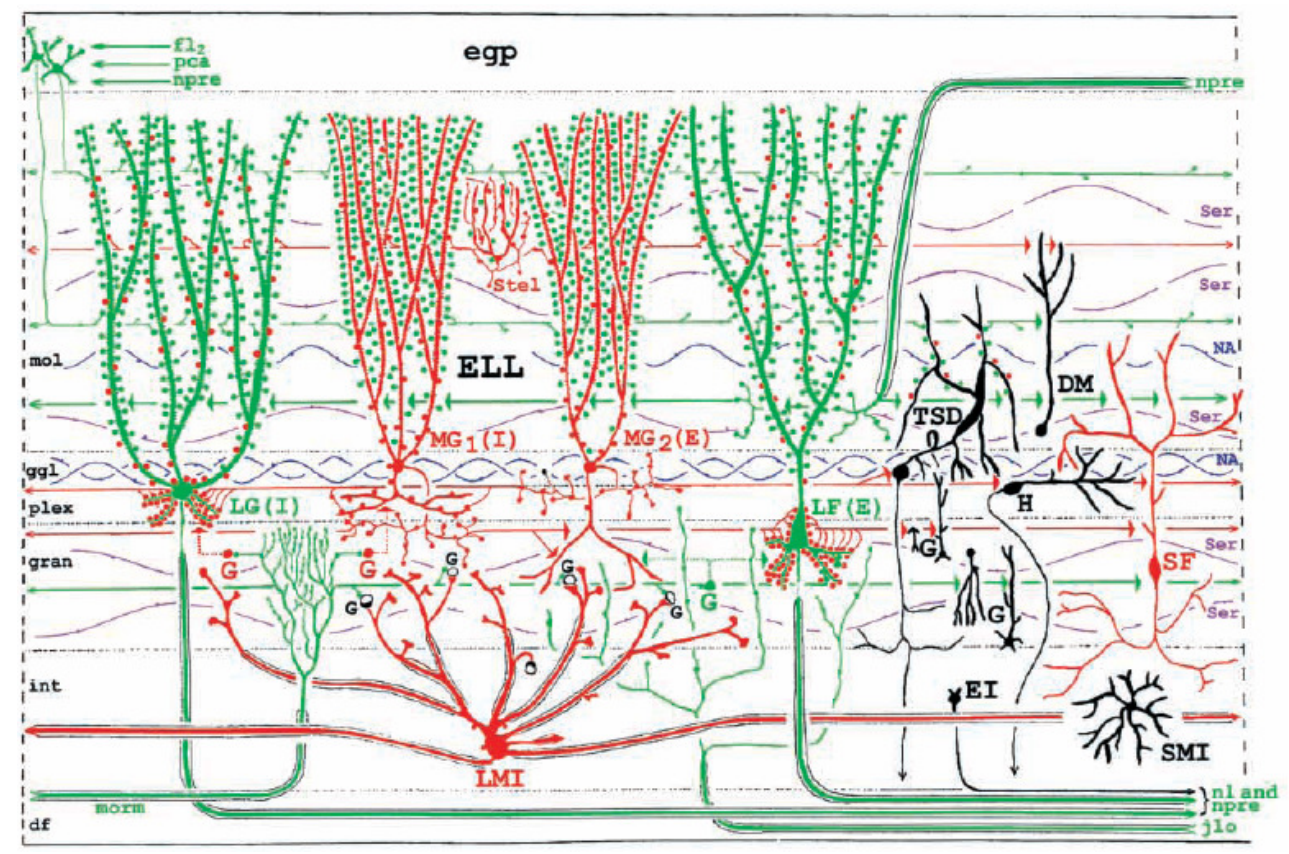

Fig. 2.5: Intrinsic circuitry of the medial zone of ELL (Meek et al. 1999)

The cells of interest to this thesis, are the MG cells, the Efferent cells, the parallel fibers, the afferent cells, and the efferent cells. The MG are the red cells in the ganglionic layer, which is title "ggl". The LG and LF cells are efferent cells in the layer titled "plex" for plexiform layer. The parallel fiber inputs are the fibers extending across the molecular layer "mol". Electrosensory inputs come through intermediate cells, 
labeled "morm" for mormyromasts (the electroreceptor type). Stelate cells are labeled "Stel," within the molecular later.

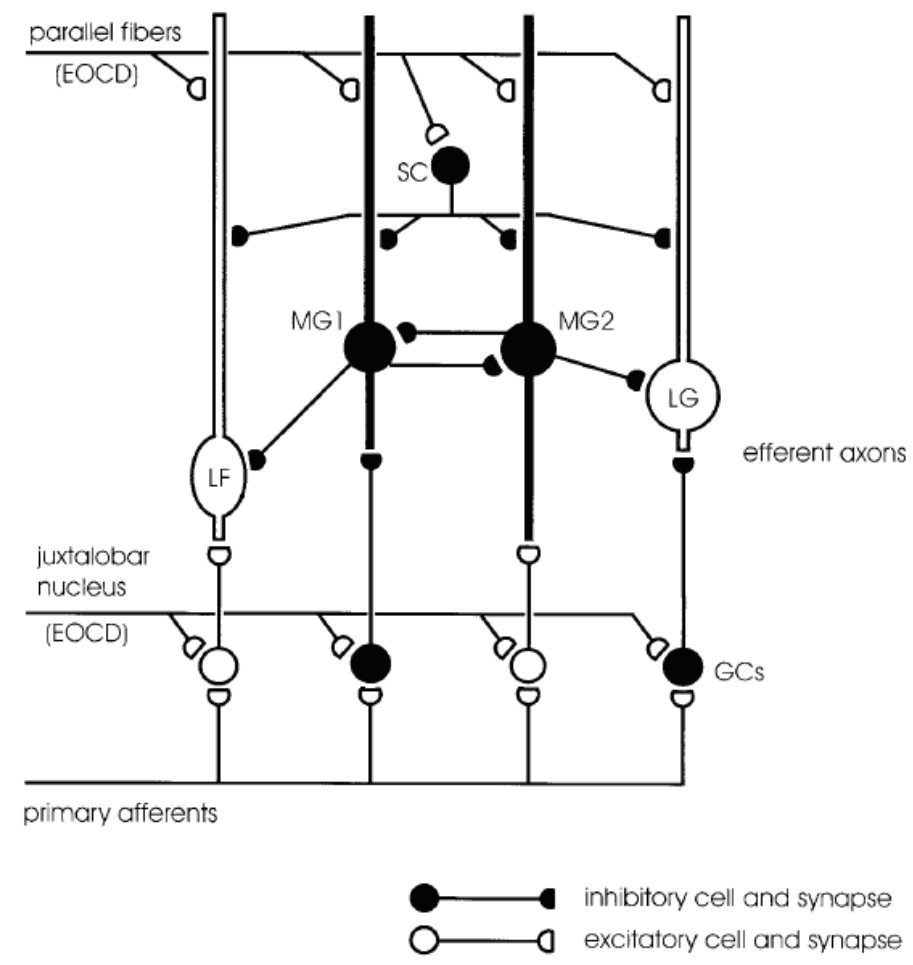

Fig. 2.6: Hypothesized functional circuit of the ELL (Han 1999)

LF and LG are 2 different types of efferent cells. GC is the granular cells, which receive afferent input. MG1 and MG2 are shown interacting. SC is inhibitory stelate cells.

Previous research on cerebellum-like structures provides a framework for understanding the adaptive filter function observed in the ELL. Fig. 2.7 highlights the four main layers found in cerebellum-like structure: sensory input layer, principal cell layer, molecular layer, and a granule layer. MG cells are the principal cell of the cerebellum-like structure found in the ELL. They are GABAergic, and have a wide dendritic arbor as typical with Purkinje-like cells. There are two types of MG cells, labeled "MG1" and "MG2" in Fig. 2.5 and Fig. 2.6. The difference between these is their input type: one gets excitatory input from granular cells, the other gets inhibitory input. The ELL receives sensory input from the granular cells, which are interneurons from electroreceptor afferents. These granular cells are depicted in Fig. 2.5 by the green cell 
on the bottom left, labeled with "morm," short for mormyromast (the electroreceptor type). This signal is passed through the principal cell layer, (the Purkinje-like MG cells). MG cells have spine-covered apical dendrites that project into the molecular layer, labeled "mol" in Fig. 2.5, and are innervated by the parallel fibers of the granule cells from the eminentia granularis posterior (EGp). These granule cells make the "granule layer" of Fig. 2.7. Parallel fibers are shown in Fig. 2.5 as the horizontal fibers in the molecular layer (mol). There are typically inhibitory interneurons labeled "stel" in Fig. 2.5 in the molecular layer, "SC" in Fig. 2.6, and the black cells of Fig. 2.7. It is through the parallel fibers that the ELL receives its predictive inputs, including proprioceptive signals, recurrent electrosensory input from higher levels, and corollary discharge signals (Bell, 2008).

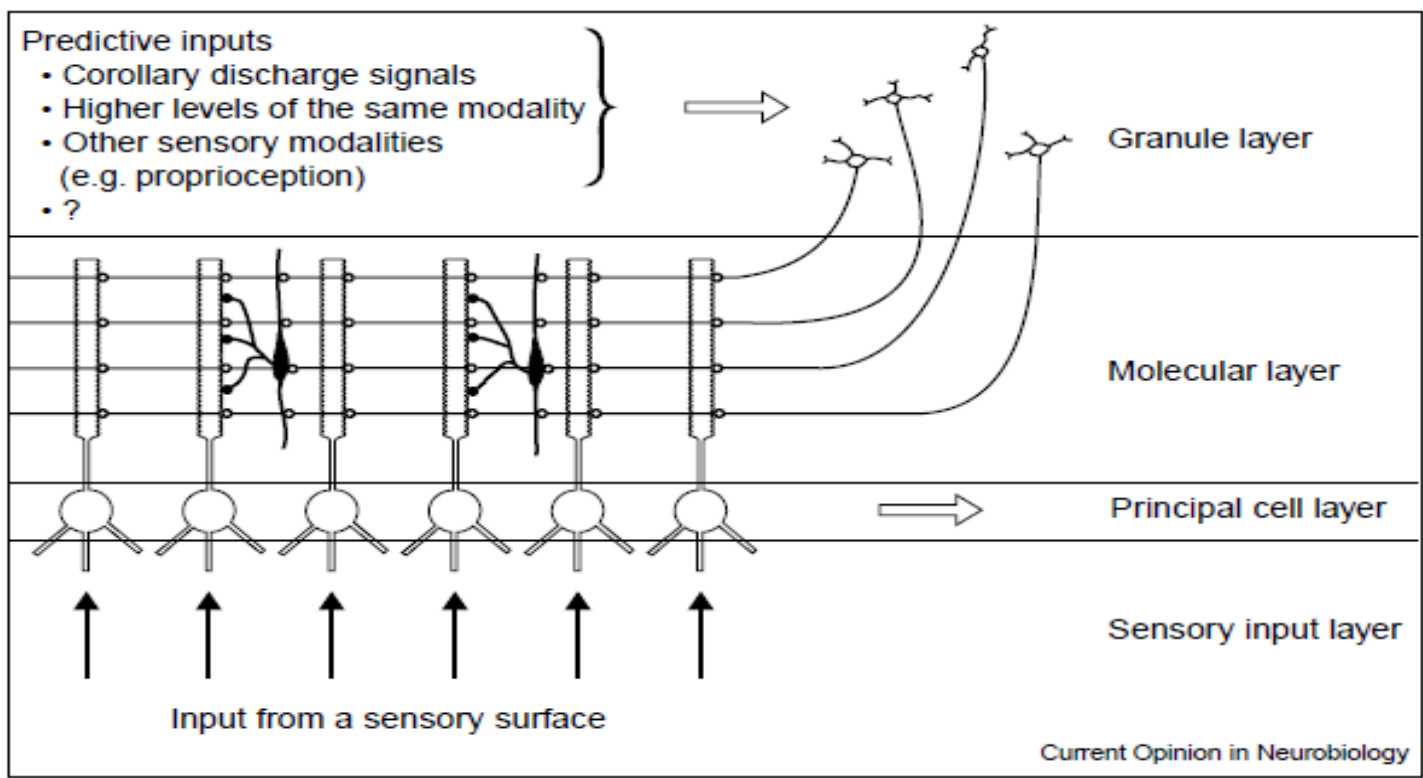

Fig. 2.7: Major Features of a Cerebellum-like Structure (Bell, 2001) 


\subsubsection{Synaptic Plasticity}

MG cells exhibit spike-timing dependent synaptic plasticity (STDP) at their parallel fiber synapses. This means that the cell's connections strength is changed based on a learning rule that depends on the relative timing of pre-synaptic and post-synaptic spikes. The learning rule used by MG cells' STDP is anti-Hebbian (Bell, Han, Sugawara, \& Grant 1997), which makes it so that the synaptic weight is reduced if a post-synaptic spike closely follows after a pre-synaptic spike has occurs. This is principle is shown in Fig. 2.8 , by the negative weight change that occurs if there is a postsynaptic spike soon after a presynaptic spike. There is an additional change in weights that is demonstrated in this figure, called "non-associative potentiation." This is a force that increases synaptic weights continuously without a dependence on spike times. This is demonstrated by the points that have positive weight change outside of the range closely following a presynaptic spike. This potentiation acts to oppose the depression caused by the antiHebbian rule because it gradually increases synaptic weights to a point at which the weight is high enough to cause a post-synaptic spike. This spike then drives the weights back down. 


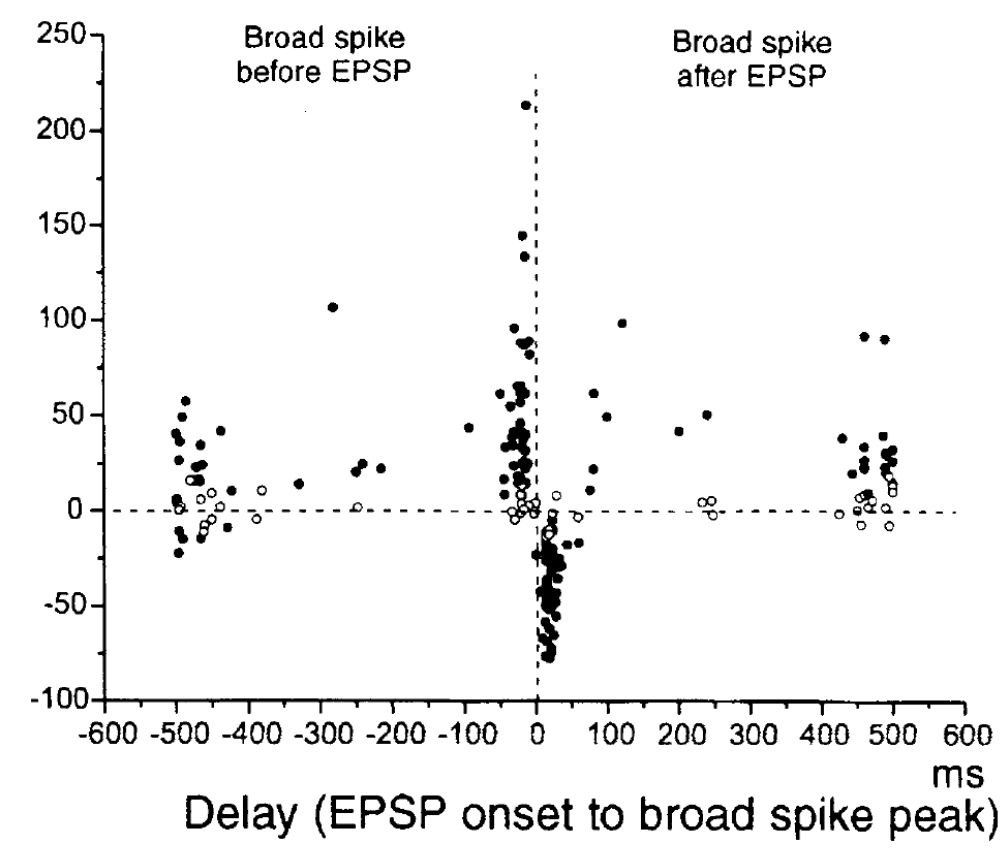

Fig. 2.8: Anti-Hebbian Plasticity displayed in MG cells (Bell, 1997)

The $\mathrm{x}$-axis is the time of postsynaptic spike (EPSP) relative to a presynaptic spike, with the presynaptic spike occurring at time 0 . Along the y-axis is the change in synaptic strength that would take place give the relative spike timing.

By changing the degree of excitation at individual MG cells, the entire circuit's activity is transformed. On a global level, the MG cells form the principal layer, which processes electrosensory input across a topographic spatial distribution of the organism's body; only local regions of MG cells receive local electrosensory input. Plasticity leads to the habituation of predictable local input in these cells after an extended period of association between signals conveyed by parallel fibers and a consistent pattern of electrosensory input. This process produces what is called a "negative image" of the electrosensory world, which is a reduced spiking potential for MG cells that transmit the sensory signals of the persistent stimuli. By creating a negative image, the ELL can removed predictable stimulus from the sensory flow due to the stimulus' reduced potency 
in triggering MG cell spikes. The negative image allows only novel input to pass through. (Bell, 2002).

\subsection{Summary}

This chapter has looked through current scientific knowledge about electroreception. It has shown that electroreception is a phenomenon that can be viewed from different perspectives. It can be viewed through its evolutionary history, through its functional behavior within an environment, through the biological structure of the fish as an organism, and through the necessary neural processes that allow for stimulus identification.

By combing these perspectives into a broader perspective that captures the structure and function of electroreception, as well as the informational processes that take place in the brain, one gets a deeper understanding of how electroreception comes into being through interactions that extend across the brain-body-environment boundaries. The biological framework has provided extensive insights into these perspectives separately, but has not brought them together into a unified framework that explains how one is connected to the other. Such a framework would be useful to explain gaps in our understanding of the ELL, which have not been able to get probed by biological techniques.

The next chapter looks to the concepts of systems science, and builds a framework for understanding electroreception. Chapter 4 describes the computational approach, which is used to create a computational model of electroreception in a way that 
brings together the ELL's structure and function. This model is then used to experiment on the ELL and look for insights that are not easy in biological experimentation. 


\section{Chapter 3}

\section{Formulation of Research Topic}

The previous chapter introduced the biological foundation for the study of electroreception. It looked at the extensively mapped anatomical components of the ELL, the activation patterns of the cells in the structure, its STDP, and its functional patterns composed of inputs and outputs to other brain regions. Unfortunately, the biological approach does not build a testable scientific model that describes how the components' interactions bring rise to the operation of the ELL as a system. This is a relevant scientific pursuit, as only by explaining how the components operate together to produce electroreception can we really understand electroreception. This chapter looks forward, by discussing possible next steps that can be taken to unifying our knowledge of the ELL's structure and function by building a model of the ELL.

Systems science provides a rich conceptual framework for understanding two main perspectives that can be taken of systems: the structural and functional perspectives. The structural perspective looks at systems as collections of interacting components that operate in such a way that behavior is generated on the level of the system as a whole. The functional perspective looks at systems as functional units that interact with other systems inside of a greater environment, or suprasystem. Systems science aims to provide scientific approaches to describing how this interaction between the structural components generates functional behavior on the level of the system, and how the resulting interaction with external systems in turn drives the internal dynamics. This 
loop-like nature between the system levels requires a broader perspective that looks to the functional features, the relevant components which underlie these functions, and integrates them into a unified model.

In this chapter, the conceptual framework of systems science is used as a foundation for describing the ELL's structure and function, and bringing them together into a unified model. Many anatomical components of the ELL have been studied as well as many electrophysiological functional patterns. By turning the systems science perspective on electroreception and the ELL, these components can be carefully sorted through and the relevant ones chosen. Successful models incorporate only the relevant components, whose mechanisms underlie a function of interest. By modeling only the relevant component, a minimal yet sufficient model is created. Section 3.1 hypothesizes about what the relevant components of the ELL's adaptive filter function are, and how they might operate together to produce the adaptive filter. Section 3.1.1 looks at interactions across brain, body, and environment and discusses the interactions that might be occurring across these systems. Section 3.2 discusses the potential of a successful minimal model test hypotheses and generating predictions about the ELL. Section 3.3 is a summary of this chapter, which discusses the goals of this thesis and how a model of the ELL can drive scientific progress in our understanding of electroreception.

\subsection{The ELL's relevant components}

By breaking up a system into its relevant components and its relevant environmental interactions, a less cluttered framework is created that allows for the construction of a minimal model that describes the system's function. The aim of this thesis is to build 
such a model of the ELL, and to do so requires identifying the ELL's relevant components and capturing their interactions through quantitative means. If the model's simulation successfully reproduces the functional patterns of interest, then it validates that the chosen relevant components are sufficient. If the patterns are not reproduced, then it suggests that the proposed model of the ELL is incomplete.

Researchers have looked to the cerebellum-like structure of the ELL as a sufficient model for producing the ELL's adaptive filter function (Bell 1999, Sawtell 2010). This structural model is adopted and tested in this thesis as the guiding structural framework. Building it requires accurate modeling of the cells that make up the cerebellum-like structure, and their interconnections. This includes MG cells, efferent cells, granular cells, and sensory afferent cells. Stellate cells are not incorporated because they do not have as strong of an affect on the ELL. Chapter 4 describes how these cells' individual activity is captured by a set of differential equations. The cerebellum-like structure and its components' dynamics are both assumptions that have to be understood as assumptions.

The model will incorporate three main input sources of data that are potentially sufficient to produce the adaptive filter function. It will incorporate the pattern of inputs from motor command areas that trigger the EOD, called the electric organ corollary discharge (EOCD). It will include inputs from proprioceptive information regarding body position (specifically tail position), and from electroreceptor afferents regarding sensory activity across an array of receptors. These inputs are all known to occur in the cerebellar-like structure of the ELL, with the EOCD and proprioceptive signals coming through the parallel fibers, and electrosensory inputs coming through sensory afferents. 
An additional important aspect of the ELL's components that takes a major role in shaping its behavior is neuronal plasticity, as discussed in Chapter 2. It is hypothesized that an ELL learns to predict repeating stimuli by creating a negative image through the anti-Hebbian STDP that has been demonstrated in MG cells (see Chapter 2, section 2.4.1). The current theory about the negative image produced in the ELL looks to the MG cells because they are the principal cell of the cerebellum-like structure and they are also the most numerous and most plastic of the ELL's cell types. If the MG cells' plasticity is sufficient to produce this function, then when plasticity at the MG cell is incorporated into a model, its evaluation should reproduce the ELL's adaptive filter function.

These relevant components are chosen for the model of the ELL constructed for this thesis based on the current hypotheses in the field and scientific evidence for their relevance. Their sufficiency will be tested after the model is created and its functional patterns simulated. If the incorporated components are sufficient as hypothesized, then when provided with representations in a simulation, the model will produce functional patterns equivalent to those of real ELLs.

\subsubsection{Across the brain, body, and environment}

As the mormyrid's body moves, the electric image along its body changes. The electric organ is in the fish's tail, and when it is on one side of the body the discharge is enhanced on one side of the fish and weakened it on the other. This change in electrical current along the different sides of the body affects the strength of electrosensory input to the ELL, and could potentially distort the detection of relevant stimuli in the environment. 
Yet, the ELL has been demonstrated to localize novel stimuli in a stable manner regardless of the fluctuations caused by bodily movement. For this to happen, the ELL must somehow remove the effects of body position from its sensory inputs in order for its outputs to be predictive of novel stimuli.

Nate Sawtell and Alan Williams describe a hypothesis in which proprioceptive inputs act to oppose the sensory consequences of tail position (Sawtell \& Williams 2008). Proprioceptive information could be used to predict changes in the input patterns that are caused by bodily movements. Through this predictive ability the ELL can adjust its excitability and adaptively filter out electrosensory stimuli that are consequences of the predicted body position. But if there are novel stimuli that were not due to body position, they would not be predicted and would still pass through the ELL's filter. Sawtell and William look at evidence about the behavior of the ELL in response to tail position, and piece the pieces together in an attempt to explain how the reliable adaptive filter might occur.

Proprioceptive input has indeed been shown to come into the ELL through the parallel fibers, and synapse onto MG cells. It has also been demonstrated that different sets of MG cells respond differently to proprioceptive input. The plot in Fig 3.1 shows the response of a particular MG cell to different tail positions, with enhanced firing frequency (EPSP rate) when the tail is on one particular side of the body. There are also MG cells that respond in the opposite manner, with more activity when the tail is on the other side. This makes it so there are certain MG cells that are more active at different tail positions. Anti-Hebbian plasticity trains upon this pattern of inputs, and adapts by reducing the degree of response to predictable stimuli as the tail moves from side to side. 
When the electrosensory input comes to the MG cells, the more active cells produce spikes more easily and train to the stimuli by creating a negative image.
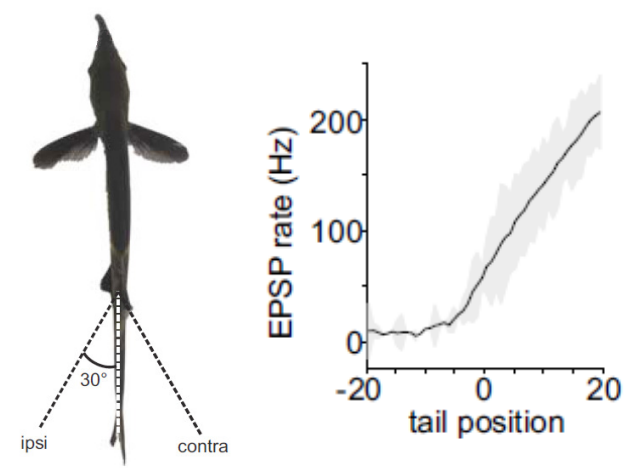

Fig. 3.1: Tail position's effect on cellular activity (Sawtell \& Williams 2008, Sawtell 2010)

Fig 3.2 demonstrates that the ELL's efferent cells produce signals that predict object location regardless of tail position. In this figure a measure of information called "SSI" is measured for afferent cells in Figure C and efferent cells in Figure D. Afferent cells carry electrosensory information prior to the MG layer filter, and efferent cells carry information after the filter. These plots show the information content of cells at different object position. In the graph labeled $\mathrm{C} 4$, information about object position is different between object only (the grey line) and object with tail movements (the black line), demonstrating that there are cells that varied noticeably with the inclusion of tail movements. The graph labeled D4 shows that the information about object position in spikes/EOD is virtually unaffected by tail movements, for the same tail movements of graph C4. This demonstrates that information content about objects when the tail is moved is different than when the tail is not moved before reaching the MG cells, and the information content is the same after passing the MG cells. This suggests that the MG cells make it so the effect of body position is negated. 

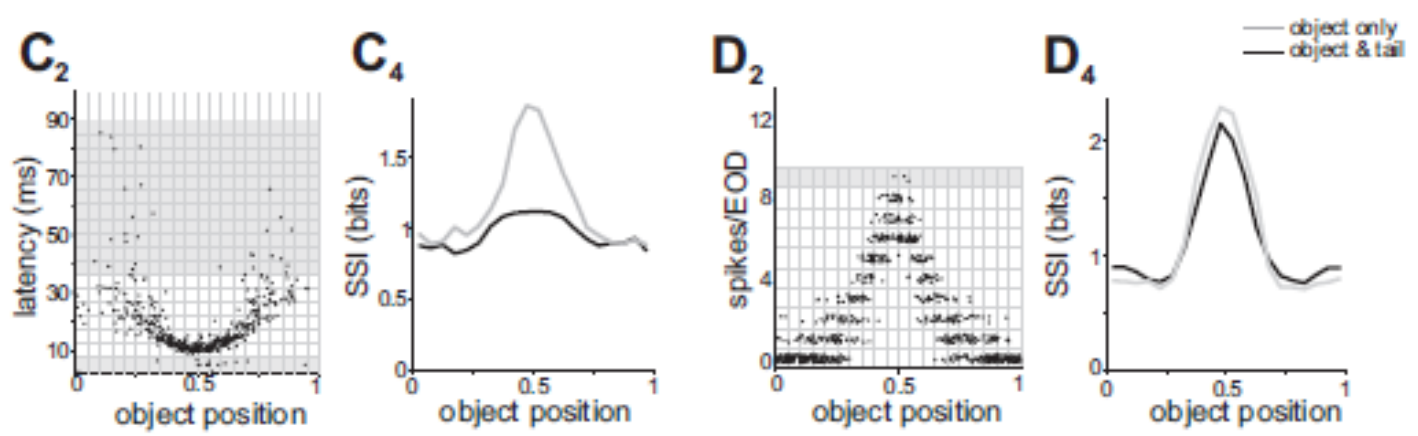

Fig. 3.2: Afferent and efferent informational measures (Sawtell \& Williams, 2008)

Systems science looks to both a system's structure and function and aims to bring them together into a unified perspective. The ELL is a system in itself made up of interacting neurons, but it also serves a function within its suprasystem. This thesis aims to bring the ELL's structure and function together by creating a model. As demonstrated by Sawtell and Williams, the ELL's inputs are affected by the electrosensory consequences of tail bending, yet it is able to filter out these consequences. A successful model would incorporate these consequences of the body and environment into a model of the ELL, and its simulation would be able to reproduce the adaptive filter of the ELL by filtering out these electrosensory consequences.

\subsection{The function of a model}

If the chosen relevant components are incorporated into a model and simulated, they will generate predictions about functional patterns of the ELL. These predictions can then be compared to data acquired from real ELLs to validate or to nullify the modeled components' sufficiency. A successful model of the ELL will capture the mechanisms 
underlying the adaptive filter function, and will generate functional patterns seen in real ELLs in simulation; this includes the adaptive filter function. By creating such a model, our scientific understanding of the ELL will be enhanced due to the confirmation of the previously untested theory regarding the cerebellum-like structure's sufficiency.

Not only can a model of the ELL be used to validate or nullify our current theories, it can also be used to generate and test hypotheses that are not easily examined through biological experimentation. A model can be used to generate predictions in different hypothetical contexts, which will point at behavior that might exist in real ELLs. If these predictions strike one as interesting, they can lead to further biological experimentation that will lead to confirmation or nullification. Simulated evidence can provide direction for further exploration.

For this thesis, the completed model is used to demonstrate how such tests can be run. There are several unknown components that are currently being investigated. These include the existence of gap junctions in the excitatory connections between the granular cells to MG2 and LF. Gap junctions are electrical synapses, which transmit signals directly to the next cells through junctions that allow an electrical current through. They have been hypothesized to exist in the ELL due to the patterns of activity observed, but have not been able to be confirmed definitively through biological tests. In the results section, the model of the ELL is simulated with and without such junctions, to test if their presence is necessary for proper function.

There is also debate about the necessity of plasticity in efferent cells. Apparent plasticity has been discussed due to the adaption shown in efferent cells, but it might also be due to plasticity at the MGs that affects efferent firing. In the results section, the 
model is used to test if efferent cell plasticity is a relevant component of the adaptive filter by testing a model with and without efferent plasticity, and also testing the effect of MG cell plasticity on efferent cells.

\subsection{Summary}

The need for synthesis was clear after discussing previous research in the background of electroreception. This synthesis would consist of a model that can bridge the gap between the relevant structure and function of the systems involved. This thesis proposes to build a model of the ELL to begin the synthesis of knowledge regarding electroreception. Systems science provides a conceptual framework for framing the ELL as system, which allows it to be modeled more easily. It suggests that we need to identify the minimal components and their connections, and identify the input that is sufficient to produce the ELL's adaptive filter behavior. This requires thinking across the brain, body, and environment as the interactions between these levels shape how the ELL behaves.

The cerebellum like structure is adopted to represent the ELL, and each of the constituent cells are fit with differential equations (described in the next chapter). Three important inputs are incorporated: electrosensory input from electrosensory afferents, the corollary discharge (EOCD), and proprioceptive inputs. Synaptic plasticity in the form of anti-Hebbian spike-timing dependent plasticity is incorporated in the MG cells' parallel fiber input, which will allow the ELL to habituate to strong sensory inputs after experience reduces the synaptic weights. The body motion's effect on electrosensory input has to be taken into account, so that when the tail is bent in one direction one side 
of the body has an enhanced electrical current and the other is reduced. MG cells that respond differently to proprioceptive input (as in Fig 3.1) have to be added to allow for training to stimuli at different tail positions.

Now that these relevant components have been considered and chosen, they need to be incorporated into a model and tested. The next chapter will look to computational neuroscience, which has developed an approach for modeling and simulating neuronal system. It will consider the different constraints required for modeling and ELL, and how they can be applied in computer code. 


\section{Chapter 4 \\ Description of Approach}

The computational approach is adopted by this thesis to create a computational model of the ELL. This approach takes the conceptual framework of systems science, and applies it to the creation of a computer model. The model uses quantitative equations to capture the behavior of the system's relevant components, and simulates their interactions by running a computer program. If the model captures the relevant components accurately, then its simulation should predict functional patterns that correspond to those seen in reality. The goal of the computational approach is to build a computer program that captures the underlying mechanisms of a phenomenon, and to use this program to generate simulations that predict functional patterns in reality. These simulated results are compared to real data to drawn conclusions about the model's sufficiency.

This chapter looks to the field of computational neuroscience, which has developed its quantitative approaches to modeling and simulating neuronal systems. After looking through a few approaches used in computational neuroscience, the chapter turns its attention to the ELL. It discusses the requirements needed to capture it in mathematical equations, and what kind of programming can be used to simulate the equations. Chapter 5 takes these technical requirements and further describes the specific steps taken to create the model of the ELL and run its simulations. 


\subsection{Computational neuroscience}

Computational modeling is an important tool for neuroscience. It helps to create models of neuronal systems that reproduce the patterns seen in reality. Modeling involves a reduction of the complexity of the real system to reveal principles of organization. These underlying principles are captured in a mathematical model that can reproduce the patterns seen in reality. By reducing complexity, the computational energy is reduced. Instead of producing a simulation that is accurate on the micro-scale, a reduced model can approximate the involved details, and simulate a much larger amount of neurons. Such models can enhance our understanding of neurophysiology by isolating the mechanisms that underlie a phenomenon, generate new hypotheses about neuronal interactions, allow us to test hypotheses, and help us develop models of neuronal learning.

The field of computational neuroscience has developed models of neuronal systems across many different levels, from the higher-level systems of neural population dynamics, such as dynamic neural fields (Spencer, Perone \& Johnson 2008) to low-level detailed models such as the Hodgkin-Huxley model (Hodgkin \& Huxley 1952). Computational neuroethology (Beer \& Chiel, 2008) looks to model neural systems along with their corresponding body and environment. Computational neuroscience has also examined and modeled plasticity, classical conditioning and reinforcement learning, central pattern generators, and more.

The most complex and accurate model of neuronal activation is the HodgkinHuxley model (Hodgkin \& Huxley 1952). This model captures multiple electrical properties of neurons, and uses them to describe how action potentials are initiated 
through multiple ionic currents across the cellular membrane, and how they propagate through the depolarization, repolarization and refractory periods. The main electrical components of the Hodgkin-Huxley model are diagrammed in Fig 4.1. These components include multiple separate currents that can pass through the membrane. The currents are show in the diagram as $I N a, I K$ and $I L$. They each have a resistance and voltage. The membrane itself has a capacitance, allowing it to hold an electric charge.

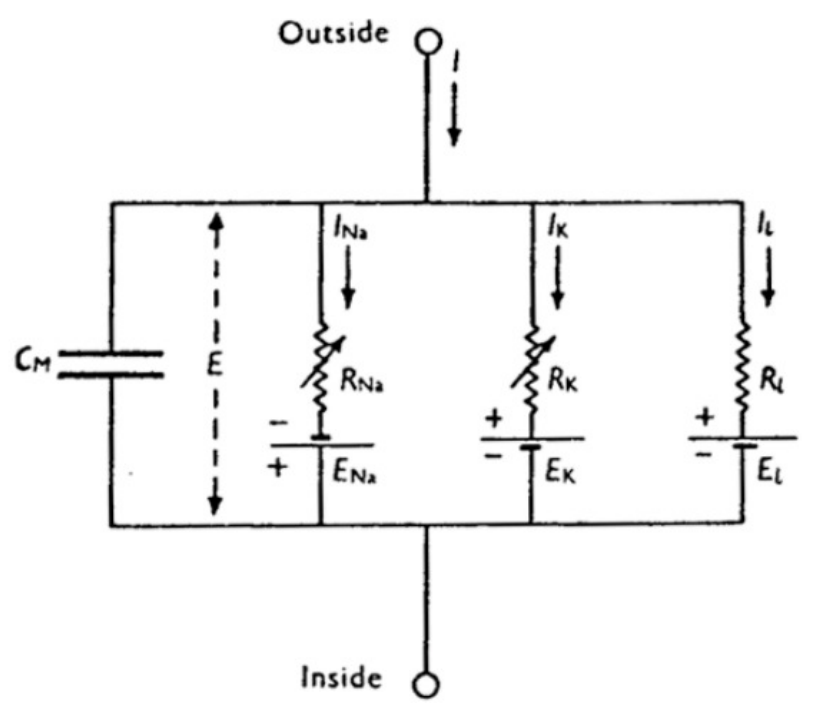

Fig. 4.1: Hodgkin-Huxley components (Hodgkin \& Huxley 1952)

Electrical circuit represents the membrane, with one side of the circuit outside of the cell, and the other inside the cell. Currents pass through from one side to the other.

In real neurons, there are multiple currents that define the change in electrical potential of the neuron. The Hodgkin-Huxley model can capture many of these currents and compute their combined effect on the electrical potential of a cell. The primary currents used in the model are the Na current, $\mathrm{K}$ current, and leak current, as shown in Fig 4.1 as $I_{N a}, I_{K}, I_{L}$. The model can also incorporate different currents, each one contributing an effect in the neurons' dynamics. The circuit's dynamics are captured in a set of differential equation that describes the change in voltage over time based on the 
effect of the separate currents and their individual gating variables. This set of equations is seen in equation set (1).

This set of equations defines the Hodgkin-Huxley model for the three currents mentioned about, and can be extended to many more currents. The top equation in the set, which defines $\mathrm{C}^{*} \mathrm{dV} / \mathrm{dt}$, is the main equation of the Hodgkin-Huxley model, and describes the change voltage at each time step. In this equation, the differences in voltage between the ion channels and the overall voltage $\left(V-V_{N a}\right)$ are multiplied by the channel's conductance $\left(g_{N a}\right)$, and summed across all the currents to obtain an overall current of the neuron at each particular time. This current is subtracted from the injected current $(I)$ to obtain the overall change in voltage $(d V / d t)$ multiplied by the capacitance $(C)$ of the neuron. Each current is assigned gating variables $(m, h$ for the Na current, and $n$ for the K current). These gating variables have differential equations that define how much current passes through at a particular time. These are shown in the bottom three equations of equation set (1).

$$
\begin{gathered}
C \frac{d V}{d t}=I-\bar{g}_{N a} m^{3} h\left(V-V_{N a}\right)-\bar{g}_{K} n^{4}\left(V-V_{K}\right)-g_{L}\left(V-V_{L}\right) \\
\frac{d m}{d t}=\alpha_{m}(V)(1-m)-\beta_{m}(V) m \quad \frac{d h}{d t}=\alpha_{h}(V)(1-h)-\beta_{h}(V) h \\
\frac{d n}{d t}=\alpha_{n}(V)(1-n)-\beta_{n}(V) n
\end{gathered}
$$


The Hodgkin-Huxley model is a very accurate model of neuronal activity because it captures the voltage of the cell due to the combined activity of multiple currents. But as a tradeoff for accuracy the model uses a large amount of variables, which require a large computational cost to be simulated. Computational modeling aims to reduce the computational cost for simulating a model by reducing the complexity of the system in a way that preserves its principles of organization. This means that if a neuron is defined with fewer variables and can still create approximate simulations of real neuronal behavior, then the model is considered successful at capturing the principles of neuronal activation. A benefit of a simplified model is that more can be simulated at once, and their interactions captured.

\subsubsection{Two-dimensional integrate-and-fire neurons}

Integrate-and-fire neurons are mathematical models of neurons that capture neurons' electrical dynamics successfully with much fewer variables than the Hodgkin-Huxley model. They are a reduction of the Hodgkin-Huxley model, which can be formulated by combining the multiple gating variables into a single variable that describes a leak current along with other currents that are assumed to be instantaneous (Izhikevich 2007). Equation (2) captures this model, with $C$ as the membrane's capacitance, $\dot{v}$ is the change in voltage, $G_{\mathrm{L}}$ is the membrane conductance, $v$ is the voltage, $v_{\mathrm{L}}$ is the leaky current's potential, and $I$ is the input current.

$$
C \dot{v}=-G_{\mathrm{L}}\left(v-v_{\mathrm{L}}\right)+I
$$


This equation can bring the voltage up to a threshold, $v_{\text {peak, }}$ after which there needs to be a reset, in which the voltage is brought back to resting potential, $c$. This rule is written in equation (3). The reset is a simplification of reality, which brings the voltage sharply down to the resting potential in a single time-step. It captures the general behavior of neurons, and saves a lot of potential variables from being needed.

$$
\text { if } v>v_{\text {peak, }} \text { then } v=c
$$

One particular form of integrate-and-fire neurons is the 2-dimensional integrateand-fire neuron (2d-IF), which reduces neuronal activity to two-dimensions: $v$, a fast voltage variable, and $u$, a slower recovery variable (Izhikevich 2007). This model is shown in equations (4). In $2 \mathrm{~d}$-IF models, a spike is initiated when the $v$-variable crosses a preset threshold, and then the two variables are reset to a baseline. There are four dimensionless parameters: $a, b, c$, and $d$. The parameter $a$ is the recovery time constant, which controls the rate of $u$ 's recovery. The parameter $b$ controls the sensitivity of recovery to fluctuations of the membrane potential. The sign of $b$ determines the model neuron is amplifying or resonant. The parameter $c$ is the voltage reset value, to which the voltage automatically shifts after reaching the threshold value, which is written as vpeak. Parameter $d$ controls the after-spike reset values for $u$. (Izhikevich 2007). $v \mathrm{r}$ is the resting membrane potential, and $v_{\mathrm{t}}$ is the instantaneous threshold potential. As in the previous integrate-and-fire model, $I$ is the input current and $C$ is the membrane capacitance. 


$$
\begin{aligned}
& C \dot{v}=k\left(v-v_{\mathrm{r}}\right)\left(v-v_{\mathrm{t}}\right)-u+I \\
& \dot{u}=a\left\{b\left(v-v_{\mathrm{r}}\right)-u\right\}
\end{aligned}
$$
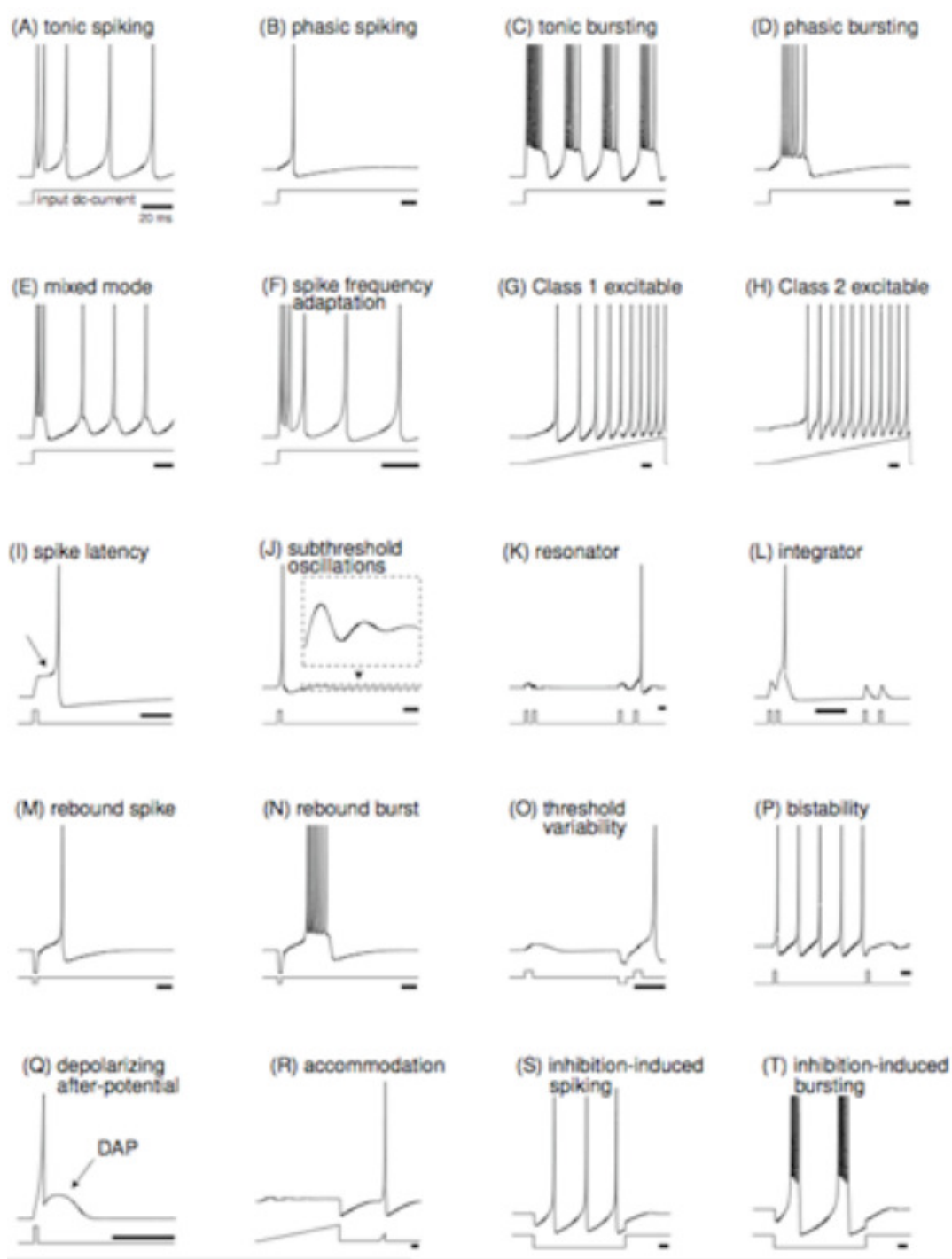

Fig. 4.2: Variety of possible patterns generated by a 2-dimensional integrate-and-fire model (Izhikevich 2007) 
An incredible amount of variety can result from this model by tuning the different parameters. This variety is illustrated upon in Fig 4.2, with twenty of the most fundamental activation patterns of neurons. With this large repertoire of possible simulated behavior, practically any desired cell's activity can be captured in a model and simulated.

\subsection{A model of electrosensation}

This thesis uses 2d-IF neurons as the model of neuronal dynamics in the ELL. To implement this model, each of the cells in the ELL has to be fit with parameters that reproduce the cells' particular characteristic activity. The cells include MG cells, efferent cells, granular cells, and sensory afferent cells. This section provides an introduction to the computational framework that is used for this thesis' model. The next chapter, Chapter 5 on Methodology, discusses all of the content introduce in this section in greater detail how.

A network structure has to be constructed in which the voltage of presynaptic neurons is converted to synaptic input current, $I$, of postsynaptic neurons. The schema of connectivity between these cells is diagrammed in Fig 2.6. To generate this network schema with $2 \mathrm{~d}-\mathrm{IF}$ neurons, each neuron's presynaptic input currents are assigned a synaptic weight that controls the effect it has on the input current of the neuron. All presynaptic currents are added up and produce the overall input current $I$. Presynaptic voltages are converted to currents by multiplying by conductance. 
The model of the ELL is aimed to examine the adaptive filter function. This requires synaptic plasticity to be implemented at parallel fiber synapses, because synaptic plasticity is the source of change that produces an adaptive filter. The connections have to reproduce the correct synaptic principles of the cells in the ELL. For example, the ELL consists of both gap junctions and chemical synapses. These different types of synapses produce different kinds of currents in postsynaptic neurons, and they have to be approximated in equations in way that maintains the ELL's behavior. Gap junctions have a much shorter time period for inward current due to the direct electrical connection between the cells. Meanwhile, chemical synapses take longer to decay because of the time required for chemical diffusion across the synapse.

MG cells have displayed anti-Hebbian spike-timing dependent plasticity at their parallel fiber synapses. This means that synaptic strength is reduced following an event in which the pre-synaptic neuron is closely followed by the action potential of a postsynaptic neuron. Fig. 4.3 demonstrates this principle with the dip in synaptic strength following an event in which a post-synaptic spike (EPSP) followed shortly after a presynaptic spike. In addition to the associative anti-Hebbian learning, MG cells also have a non-associative weight change that takes place at these synapses. This non-associative change has conductance increased when there is no post-synaptic spike. It is demonstrated in Fig. 4.3 by having the positive weight change in all times other than shortly after the pre-synaptic spike. This can be reproduced in the computational model by comparing the pre and post synaptic spikes of the MG cells, and changing synaptic weight accordingly. 
MG cells have been shown to fire in two different kinds of spike patterns: Broad spikes and narrow spikes. The narrow spike originates in the axon and has a lower threshold. Broad spikes originate in the dendrites and have a higher threshold. The narrows spikes carry information to the next cells, and broad spikes trigger associative depression at the parallel fiber synapses (Roberts 2000). These different spike patterns have to be incorporated into a computational model for associative plasticity to function properly.

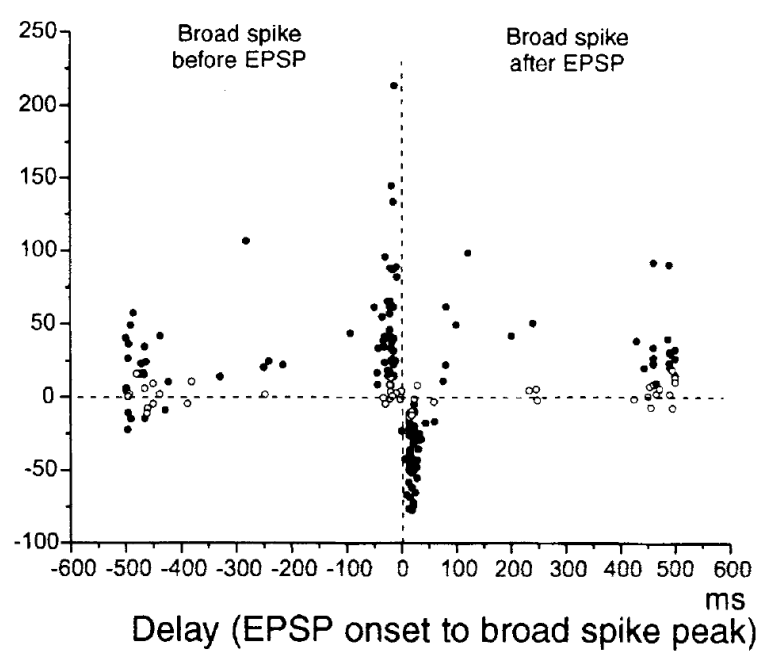

Fig. 4.3: Anti-Hebbian STDP in MG cells (Bell, 1997)

The $\mathrm{x}$-axis is the time of postsynaptic spike (EPSP) relative to a presynaptic spike, with the presynaptic spike occurring at time 0 . Along the $y$-axis is the change in synaptic strength that would take place give the relative spike timing.

External inputs from the corollary discharge, proprioceptive regions, and electroreceptors also need to be modeled correctly and fed into the correct cells through their input currents, $I$, in a way that matches reality. Each of these inputs has its own characteristic behavior that has to be captured in computer code. 


\subsection{Simulation}

Quantitative modeling of the system is only one part of the computational approach. It is also necessary to capture the mathematical model in computer code, and simulate it in different scenarios by adjusting parameters. The ELL model described in this thesis is programmed and simulated in Matlab. Matlab is a computing environment that is used to solve problems through the manipulation of data in the form of vectors and matrices. In addition to supplying built-in functions, Matlab allows users to create their own functions to manipulate data. Such user-generate functions can include differential equations such as those of the $2 \mathrm{D}$-IF model. A user can program the equations along with parameters into a function, and then prompt Matlab to compute the voltages across time. These can then be plotted using Matlab's plot function to create a visual depiction of the neuron's activity.

Once the model of the ELL is programmed by writing all the respective equations and parameters discussed in the previous section into a computer code, its activity can be simulated by running the equations across time and saving the values. Inputs will automatically be provided to the different cells through the variables that are updated by the program, and synaptic plasticity will take effect whenever called by the program. When the simulation's results are plotted, they will produce a graph the ELL's behavior as predicted by the model. This graph can be compared to data acquired from real ELLs.

If the tendencies seen in real ELLs are predicted by the computational model, then it confirms the model's accuracy. If the tendencies are not reproduced, then further exploration will be required to complete the model and produce the desired effects. This is process inherent in computational modeling, which leads to a model's eventual 
success. Once a successful model is created, it can be used to study the ELL's particular ability to process information about its environment. It can also be extended to a more general level in order to understand basic principles of sensory processing in biological system.

\subsection{Summary}

The computational approach studies systems by examining their structure, and reducing their complexity to relevant components. These components are quantitatively modeled and put in a computer program to simulate their behavior. A successful model runs a simulation that reproduces patterns of behavior seen in reality, and does so with minimized computational costs. A failed simulation does not reproduce the data observed in the real system, and points to deficiencies of the theory with which the model was built. This elicits a continued search for a working theory.

For this thesis, the computational approach is used to model the ELL in order to study its adaptive filter function. The neurons theorized to be relevant are captured in 2D-IF neuron models and connected into their cerebellum-like network structure by converting presynaptic voltages into postsynaptic currents. This must be done using the correct synaptic principles that capture the differences between gap junctions and chemical synapses. And further, synaptic plasticity must be included so that the MG cells inputs' weights are changed as a consequence of spike-timing.

If the ELL's structure and local dynamics are accurately captured by the theorized relevant structure, then a simulation of the model should reproduce global behavior as seen in real ELLs. If this accuracy is demonstrated by comparing simulations with real 
experimental data, then our scientific knowledge of the ELL's relevant structure is confirmed. It will demonstrate that the cerebellum-like structure along with its described inputs and plasticity at the MG cells is sufficient to produce the adaptive filter function and describe the behavior recorded in real ELLs. If the simulation does not reproduce experimental data, then the model is deemed insufficient. 


\section{Chapter 5}

\section{Methodology}

This chapter describes the technical details that were involved in constructing the computational model of the ELL. This builds upon the computational approach discussed in the previous chapter by focusing the general principles of computation towards the particular quantitative formulations needed to capture the dynamics of the ELL. For the model described in this thesis, a previous model of the ELL, created by Patrick Roberts and Deniz Kusefoglu in 2009, was extended to capture a broader range of functional patterns that are studied in the ELL. This chapter begins by describing the previous model's basic network structure, and how its cells' activity was captured with 2D-IF neurons. After the mathematical quantification of these cells and their connections are described, the chapter looks at the simulation results of this previous model and discusses their limitations in modeling the ELL.

Next, the chapter addresses the additions made in this thesis project, which extend the range of functional description made possible by the computational model. These additions include a corollary discharge, synaptic plasticity, proprioception, body motion effects, efferent cells, the ability to run multiple runs through a longer time scale, and multiple new types of plots to visualize the model's activity. Each contribution was programmed separately and tested separately to ensure that they worked properly on their own before being incorporated with the other components. The additions are described individually (as they were programmed) and also described by how they were 
incorporated in the model as a whole. The next chapter on results focuses on the simulated patterns that are generated by the extended model, and compares them to data found in experiments on real mormyrids.

\section{1 The Roberts-Kusefoglu model}

The Roberts-Kusefoglu model of the ELL is a network, diagrammed in Fig. 5.1, of interconnected 2D-IF neurons that were individually fit to reproduce the electrical behavior of three different cell types in the ELL: afferent cells, granular cells, and MG cells. In this model, the network is stimulated by a simulated external electric field, shown in Fig 5.1 as "electrosensory, spatiotemporal pattern," which depolarizes the afferent cells. Afferent cells make electrical synapses onto granular cells and combine with excitatory chemical inputs from the JLN's (juxtalobar nucleus) corollary discharge signal to determine the timing and duration of granular cells bursts. Granular cells make GABAergic synaptic connections onto MG1 cells and electrical synaptic connections onto MG2 cells. Excitatory inputs from parallel fibers onto MG cells combine with granular cell inputs and mutual inhibition between the two different types of MG cells. This figure can be compared with Fig. 5.2 on the hypothesized functional circuit of the ELL. Lacking in the model are efferent cells (labeled as LF and LG in the functional circuit) and stellate cells (SC).

The different cell types of this model have different characteristic functions of activity, which were captured by $2 \mathrm{~d}-\mathrm{IF}$ models. In order to reproduce the cell's characteristic activity, the $2 \mathrm{~d}-\mathrm{IF}$ model was fit with different parameters. Some of the included cell types display variations of stereotypical spike patterns in real ELLs: 
granular cells can produce $2,3,4,5$, and 6-spike bursts and afferent cells produce $2,3,4$ spike bursts. These different spike patterns were separately encoded through different parameters, shown in Table I and Table II. MG1 and MG2 cell activity was captured with the parameters of Table III.

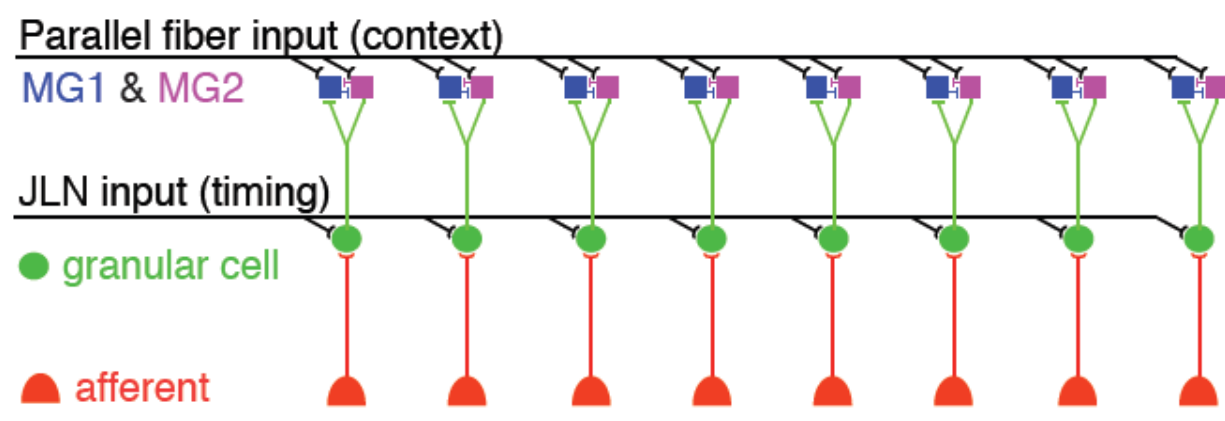

\section{Electrosensory, spatiotemporal pattern}

Fig. 5.1: The Roberts-Kusefoglu model (Roberts 2010)

Network structure of the Robert-Kusefoglu model. Electrosensory pattern comes in through the afferent cells and propagates through the arrays of cells.

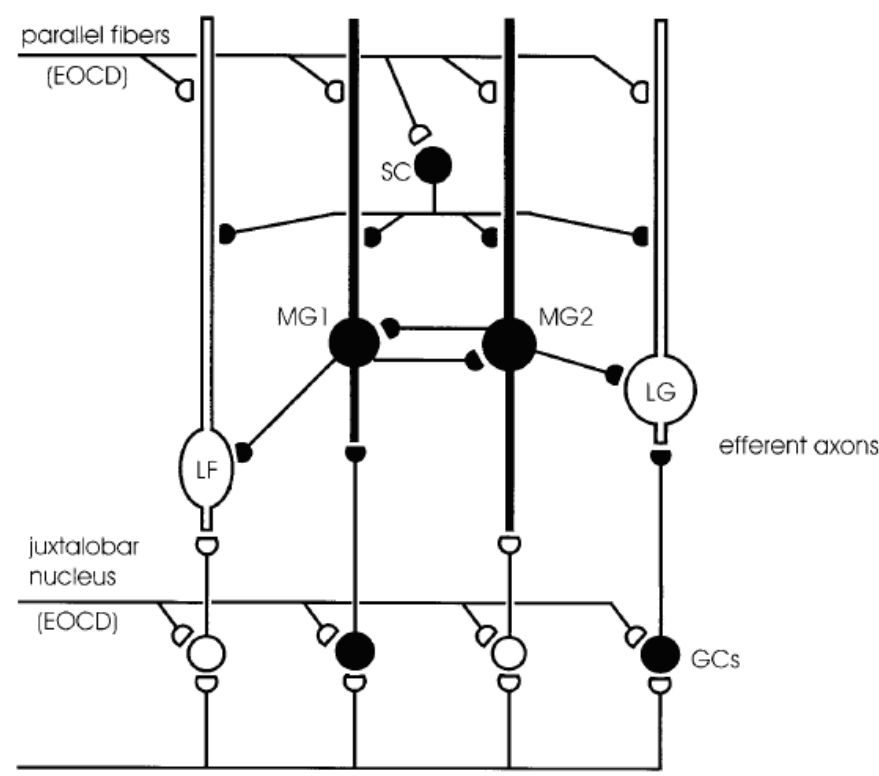

primary afferents

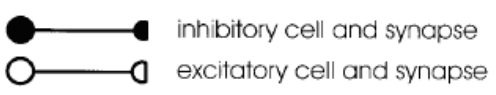

Fig. 5.2: Hypothesized functional circuit of the ELL (Han 1999) 
These parameters were used in a 2D-IF model. As described earlier, the parameter $a$ is the recovery time constant, which controls the rate of $u$ 's recovery. The parameter $b$ controls the sensitivity of recovery to fluctuations of the membrane potential. The parameter $c$ is the voltage reset value. The parameter $d$ controls the after-spike reset values for $u . \quad v_{\mathrm{r}}$ is the resting membrane potential. $\quad v_{\mathrm{t}}$ is the instantaneous threshold potential. $I$ is the input current. And $C$ is the membrane capacitance. When the 2 d-IF model is simulated with the parameters as assigned in Tables I, II, and III, it produces the activity patterns shown in Fig. 5.3, Fig. 5.4, and Fig. 5.5 respectively. Fig 5.3 and 5.4 show the 3-spike variation of the granule and afferent cells. Fig 5.5 shows the MG cell activity. These are approximations of the activity patterns seen in real ELLs.

$$
\begin{aligned}
& C \dot{v}=k\left(v-v_{\mathrm{r}}\right)\left(v-v_{\mathrm{t}}\right)-u+I \\
& \dot{u}=a\left\{b\left(v-v_{\mathrm{r}}\right)-u\right\} \\
& \text { if } v>v_{\text {peak, }} \text { then } v=c, u=u+d
\end{aligned}
$$


Table I: Parameters for granular cells, producing 2-6 spike bursts

\begin{tabular}{|l|l|l|l|l|l|l|l|l|l|}
\hline & $\mathrm{a}$ & $\mathrm{b}$ & $\mathrm{c}$ & $\mathrm{d}$ & $\mathrm{v} \_r$ & v_t & $\mathrm{C}$ & $\mathrm{k}$ & v_peak \\
\hline 2-spike & 0.05 & -5.0 & -5 & 100 & -70 & -00 & 250 & 1.0 & 30 \\
\hline 3- spike & 0.05 & -5.0 & -5 & 100 & -70 & -00 & 250 & 1.0 & 20 \\
\hline 4- spike & 0.05 & -5.0 & -5 & 100 & -70 & -00 & 250 & 1.0 & 10 \\
\hline 5- spike & 0.05 & -5.0 & -5 & 100 & -70 & -00 & 250 & 1.0 & 8 \\
\hline 6- spike & 0.05 & -5.0 & -5 & 100 & -70 & -00 & 250 & 1.0 & 5 \\
\hline
\end{tabular}

Table II: Parameters for afferent cells, producing 1-4 spike bursts

\begin{tabular}{|l|l|l|l|l|l|l|l|l|l|}
\hline & a & b & c & d & v_r & v_t & C & k & v_peak \\
\hline 1- spike & 0.05 & -10.0 & -40 & 200 & -62 & -40 & 50 & 1.0 & 20 \\
\hline 2- spike & 0.05 & -10.0 & -35 & 200 & -62 & -40 & 50 & 1.0 & 20 \\
\hline 3- spike & 0.05 & -10.0 & -32 & 200 & -62 & -40 & 50 & 1.0 & 20 \\
\hline 4- spike & 0.05 & -10.0 & -30 & 200 & -62 & -40 & 50 & 1.0 & 20 \\
\hline
\end{tabular}

Table III: Parameters for MG cells

\begin{tabular}{|l|l|l|l|l|l|l|l|l|l|l|}
\hline Cell type & a & b & c & d & v_el & v_r & v_t & C & k & $\begin{array}{l}\text { v_pea } \\
\text { k }\end{array}$ \\
\hline MG1 & 0.0 & -5.0 & -50 & 0.0 & -80 & -70 & -20 & 5 & 0.2 & 0 \\
\hline MG2 & 0.0 & -5.0 & -50 & 0.0 & -20 & -79 & -20 & 5 & 0.2 & 0 \\
\hline
\end{tabular}




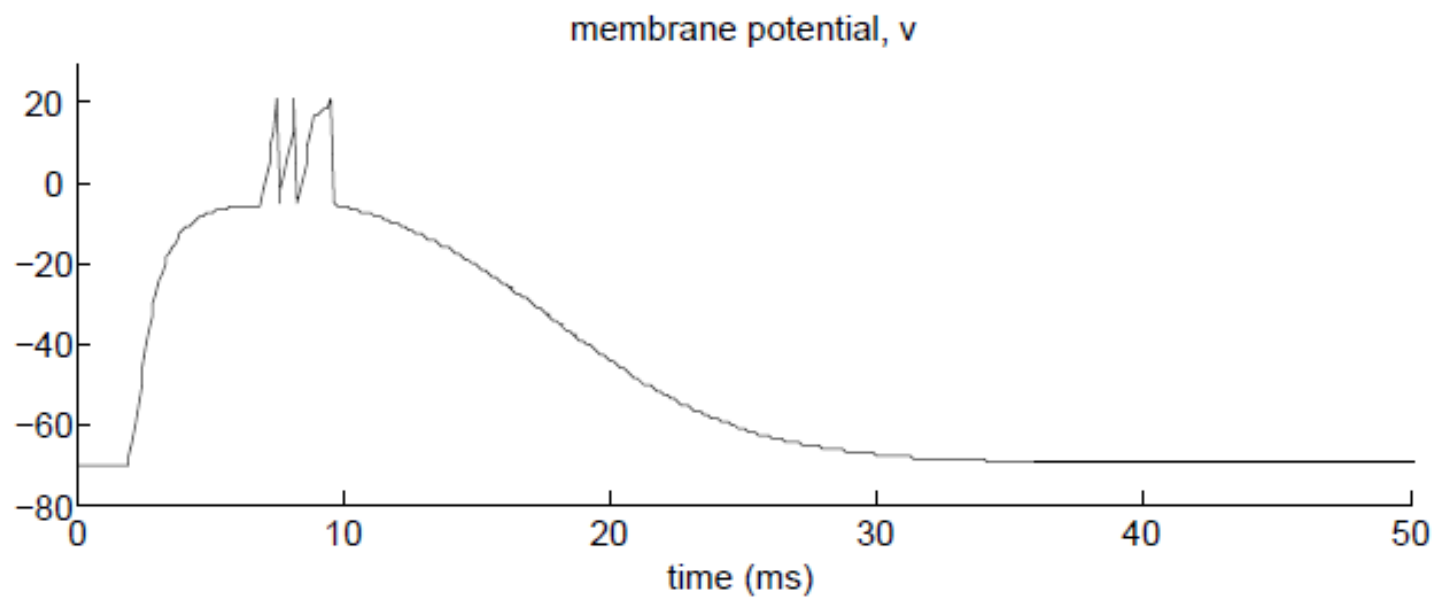

Fig. 5.3: Granule Cell Activity resulting from the 2d-IF parameters of Table I

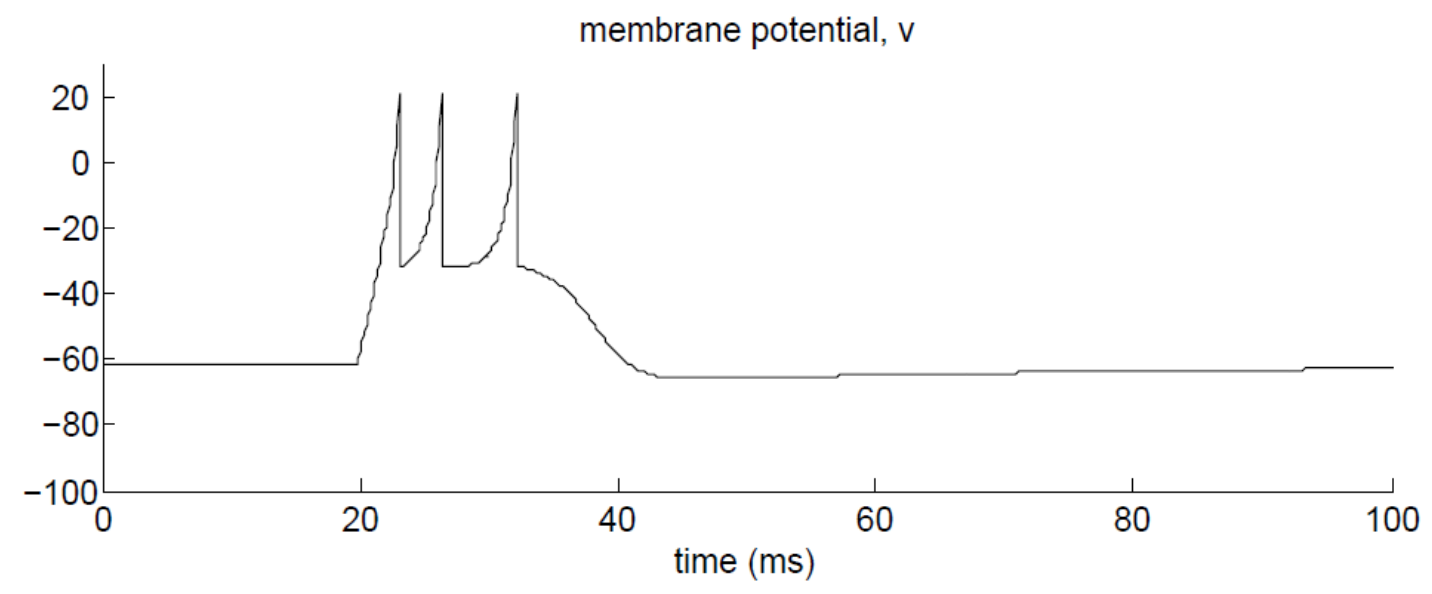

Fig. 5.4: Afferent Cell Activity resulting from the 2d-IF parameters of Table II

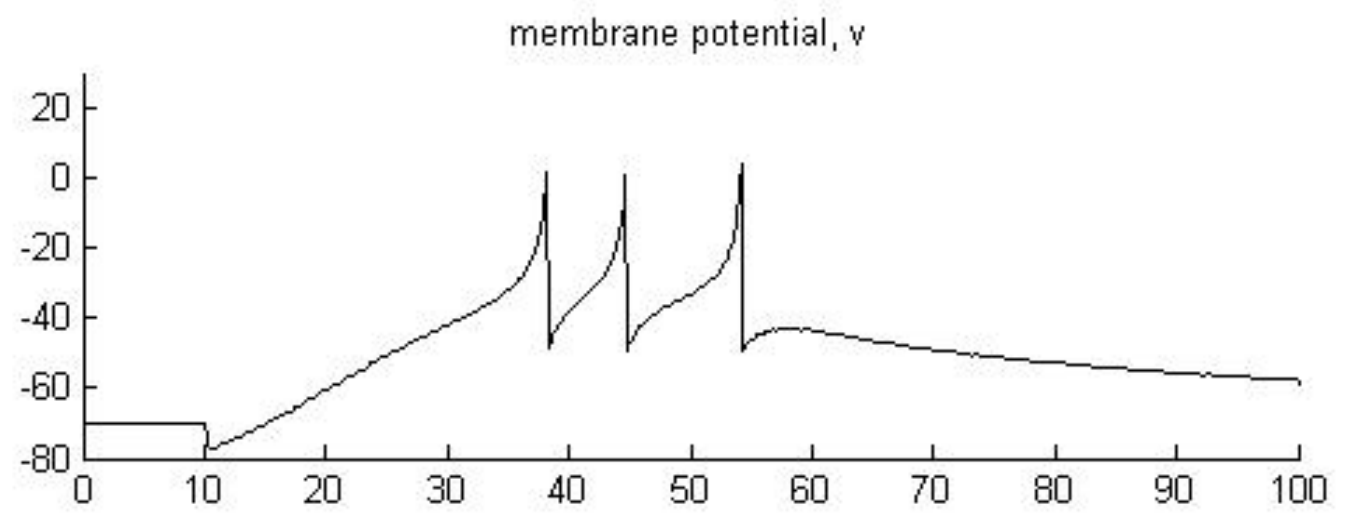

Fig. 5.5: MG Cell Activity resulting from the 2d-IF parameters of Table III 
In order to calculate the total input that each postsynaptic cell gets, presynaptic cell voltages are converted to individual electrical currents and added up into a total input current. This process was described in section 4.2. An example of the computer program used to compute the total input is show below in equation (5). This equation was used to compute the total input for MG cells, and it has to be computed at each time step throughout a simulation. The total input is made up of three inputs: parallel fiber input, electrosensory input from granular cells, and input from the opposite type of MG cell (MG1 gets inhibited by MG2, and MG gets inhibited by MG1). Each presynaptic cell's voltage is converted to an input current by finding the electrical difference between the cell's current voltage and its resting voltage, and then multiplying that difference by a conductance value.

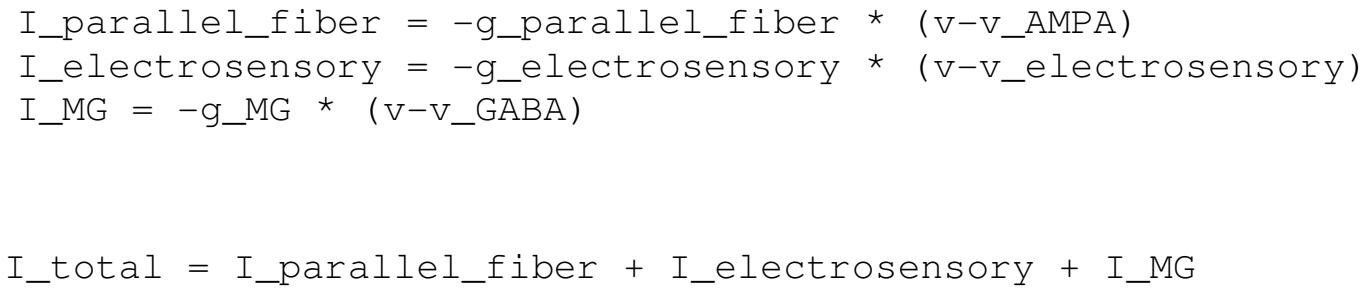

This input becomes the "I" of equation (4), and is used to compute the cell's voltage at each time step. The input current continues to fluctuate throughout a simulation because the inputs' voltages fluctuate as a result of the unfolding dynamics. This current drives the activity of cells. Resulting dynamics are simulated by calculating the values at each time-step, and then recorded in a list by the computer program. Recorded values can then be visualized to show how the cells behaved throughout the simulation in response to a particular input pattern. Fig. 5.6 is a visualization of an R-K 
model simulation that shows spike times along the $\mathrm{x}$-axis, and cell number along the $\mathrm{y}$ axis. A spatio-temporal input that consisted of a strong stimulus around skin location \#50 was injected to the afferent cells. Afferent dynamics unfolded, leading to input to the granular cells. This then propagated to the MG cells. As a result of the connectivity and the individual cell's parameters, the model produced these particular spike times.
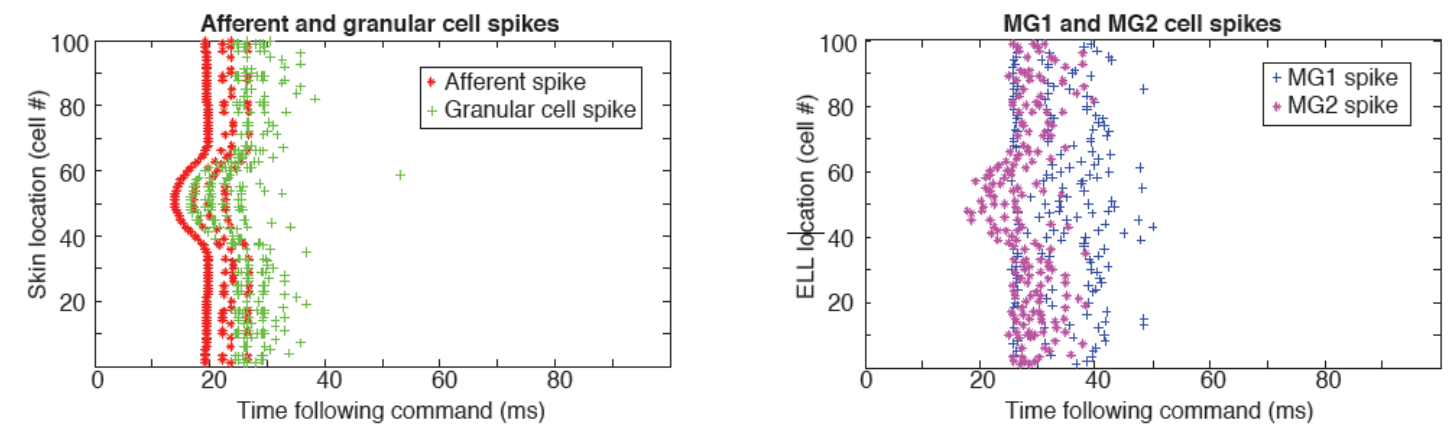

Fig.5.6: Cells' spike times in response to Gaussian input (Roberts 2010)

This figure, 5.6, demonstrates the basic functional ability of the R-K model. It highlights how the model can capture the four cell types' behavior across a single run (single experience of stimuli). The model does not incorporate synaptic plasticity, so if it was tested across multiple runs it would produce the same exact dynamics every time. This is not an accurate depiction of the ELL, because the ELL has been shown to change between trials due to plasticity. Specifically, an anti-Hebbian form of spike-timing dependent plasticity is an essential component that allows the ELL to execute its adaptive filter function and remove the signal of reoccurring electrosensory inputs from its efferent cells' outputs. Without this plasticity, a computational model of the ELL can not be used to examine the adaptive filter function. 
The R-K model also does not incorporate the effects of body position, and the proprioceptive input which acts to negate the effects of body position. One of the ELL's primary functions is to remove the effect of body positions (Sawtell \& Williams 2008). If a computational model was to examine how the ELL performs this function, it is necessary to incorporate the effect of body position on the pattern of electrosensory input that propagates through the ELL. It also needs to incorporate a realistic formulation of proprioceptive input through the parallel fibers, onto which synaptic plasticity can train to predict and remove the effects of body position.

Another necessary addition to the R-K model is efferent cells. These are shown in Fig 5.2 by the two types of efferent cells: LF cells and LG cells. Efferent cell activity has been studied extensively through neurophysiology due to their importance in the ELL's function as the primary output source of the processing that takes place in the ELL. Their activity needs to be modeled with a 2D-IF model neuron, and it needs to be connected with the appropriate synaptic inputs.

\subsection{Additions}

These limitations to the Roberts-Kusefoglu model were addressed in the model created for this thesis. Additions include an updated corollary discharge input that receives more accurately modeled individual inputs throughout the simulation rather than a continuous input as done previously. Also added is synaptic plasticity at the MG cells, which when combined with the coarser EOCD allow the model to match timing between the presynaptic and postsynaptic spikes created in response the EOCD and electrosensory 
inputs and apply an anti-Hebbian spike-timing dependent plasticity learning rule that reduces the affect of a previous stimulus on the probability of a post-synaptic spike.

Proprioceptive inputs and the effects of body position were also added. The combination of proprioceptive input and the body position's effect on sensory stimuli allows the model to simulate how the ELL removes the effects of body position from its sensory signal. Efferent cells make the final addition to the structure of this ELL model. This addition allows further modeling of the ELL's behavior, which can be compared to data from real ELLs. Efferent cells provide the primary output signal of the adaptive filter, which has predictable input removed and carries only novel input. By adding these cells to the model, the adaptive filter function can be studied.

\subsubsection{Corollary discharge}

The corollary discharge (EOCD) is incorporated in the model by computing a parallel fiber conductance at each time step. The EOCD is composed of input from multiple cells that activate at different times following the onset of the electric discharge. These cells make individual synapses onto the MG cell, and each contributes a small current that adds up in the post-synaptic MG cell. These connections are the primary synapses that display plasticity in the ELL.

In order to simulate this discharge, 100 input currents were simulated: one discharging per millisecond for a run of 100 milliseconds. An individual current is shown in Fig. 5.7. The array of 100 cells has each cell produce this activity pattern one millisecond apart, and at each time step the total current is added, producing a distribution like that of Fig. 5.8. This total current represents the combined effect that 
the cells that transmit signals to MG cells following the discharge. The activity shown in Fig. 5.8 does not have synaptic weight incorporated in its computation. In reality, synaptic weights would apply at every one of the individual synapses, making some cells have more of an effect than others. This would distort the overall EOCD across time.

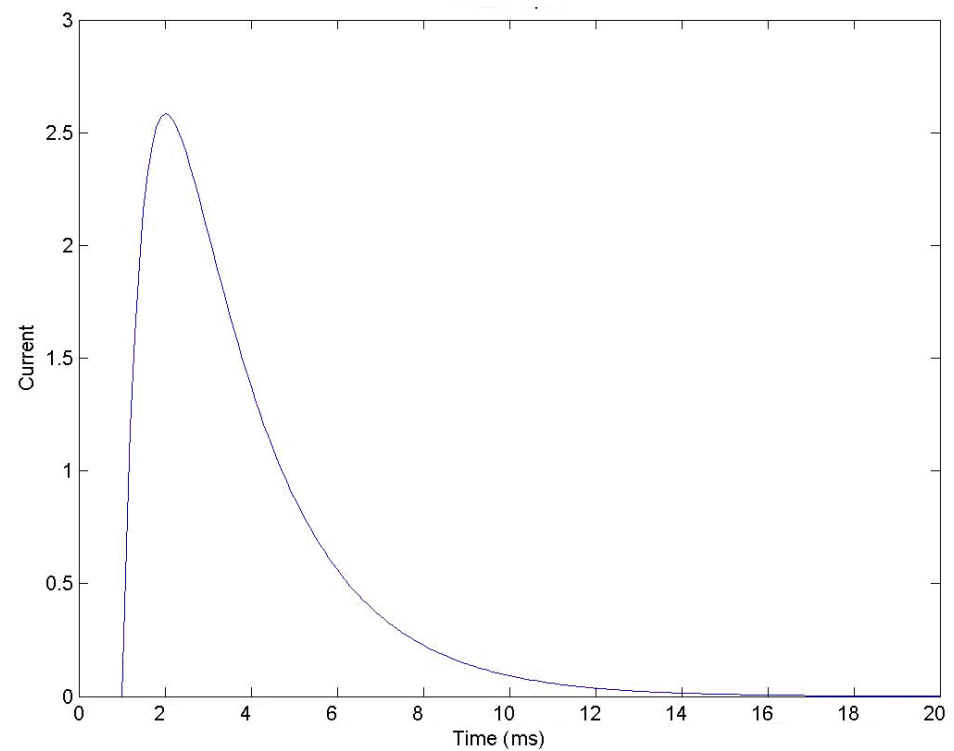

Fig. 5.7: A single parallel fiber input

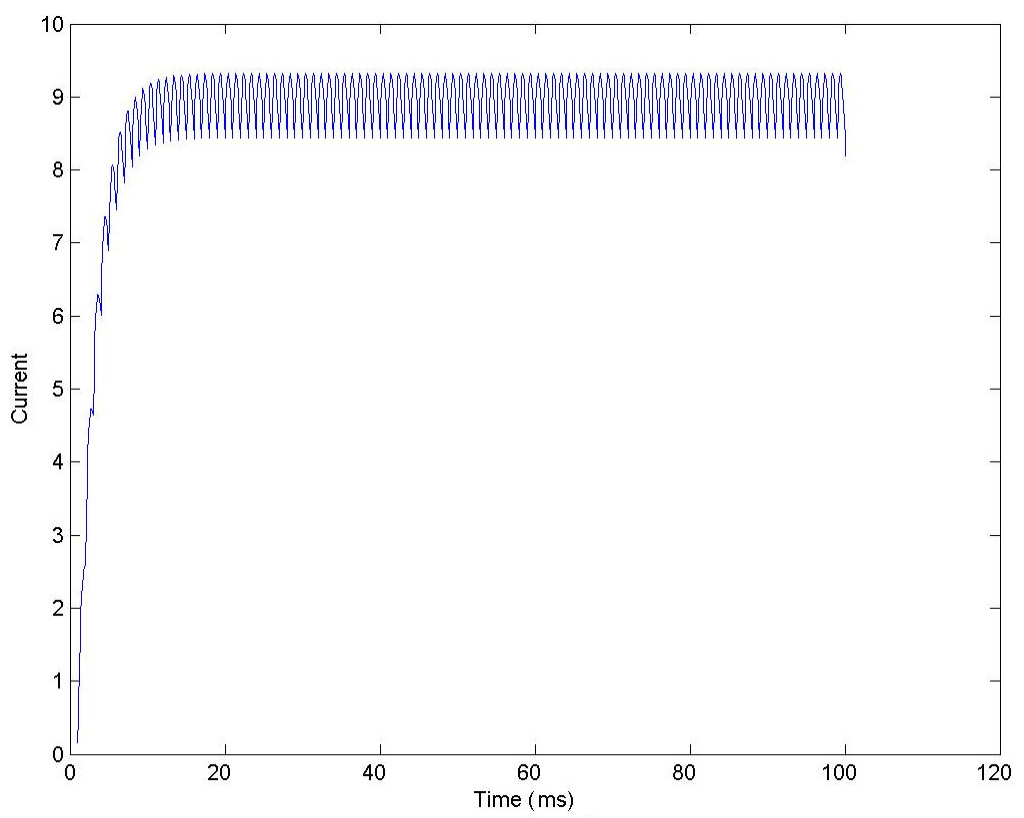

Fig. 5.8: Total current, consisting of added up parallel fiber inputs from EOCD 


\subsubsection{Synaptic plasticity}

Anti-Hebbian spike-timing dependent plasticity has been demonstrated at the parallel fiber inputs to the ELL's MG cells (Bell, 1997). This makes it so if a post-synaptic spike occurs soon after a pre-synaptic spike; the synaptic weight between those two cells is reduced, as shown in Fig. 4.3. In Fig. 4.3 the presynaptic spike (EPSP) occurs at time zero, and the $\mathrm{x}$-axis measures the time before or after the presynaptic spike at which the post synaptic (broad spike) occurs. The y-axis shows the change in weight that occurs at that timing difference. Most importantly, if the postsynaptic spike occurs shortly after the presynaptic spike, synaptic weight is reduced significantly. With an increased delay, the weight change is less significant and after a longer period of time the weight change is positive.

The change of weights that occurs over time in MG cells has to be computed in a computational model, and updated weights have to be implemented in every time-step of the simulation. In this thesis, this was done by modifying a model of plasticity in the form of equation set (5). The equation set is illustrated graphically in Fig. 5.9, with the dashed red line at the value of ltp_nonassociative, the dashed black line showing the value of $l t d$, and the solid blue line showing the two values combined. The equations use a value called $l t d 0$ (long term depression " 0 "), which is set to a maximum value as soon a presynaptic spike occurs, and then decays with each following time step. The degree of decay is one tenth the value of the maximum ltd, making it so ltd0 is back to a value of zero within 10 time steps. The actual value of potential weight change is ltd (long term depression), which is computed using a time constant, tau, that buffers the effect of ltdo. As mentioned earlier, ltd is shown in Fig. 5.9 as the dashed black line. The weight 
change is applied as soon as a postsynaptic spike occurs, shown in equation (5) as "if (postsynaptic spike)." This weight change sets the conductance (g_max) to its previous value plus a nonassociative potentiation (ltp_nonasso) minus the previously computed $l t d$, which reduces the total weight. The nonassociative potentiation is added at every time step regardless of a post-synaptic spike. This makes it so the weights decrease if a post-synaptic spike occurs soon after a presynaptic spike, but otherwise the weights increase. The blue line in Fig. 5.9 demonstrates how the weight change is negative shortly after a presynaptic spike, and positive after a longer delay.
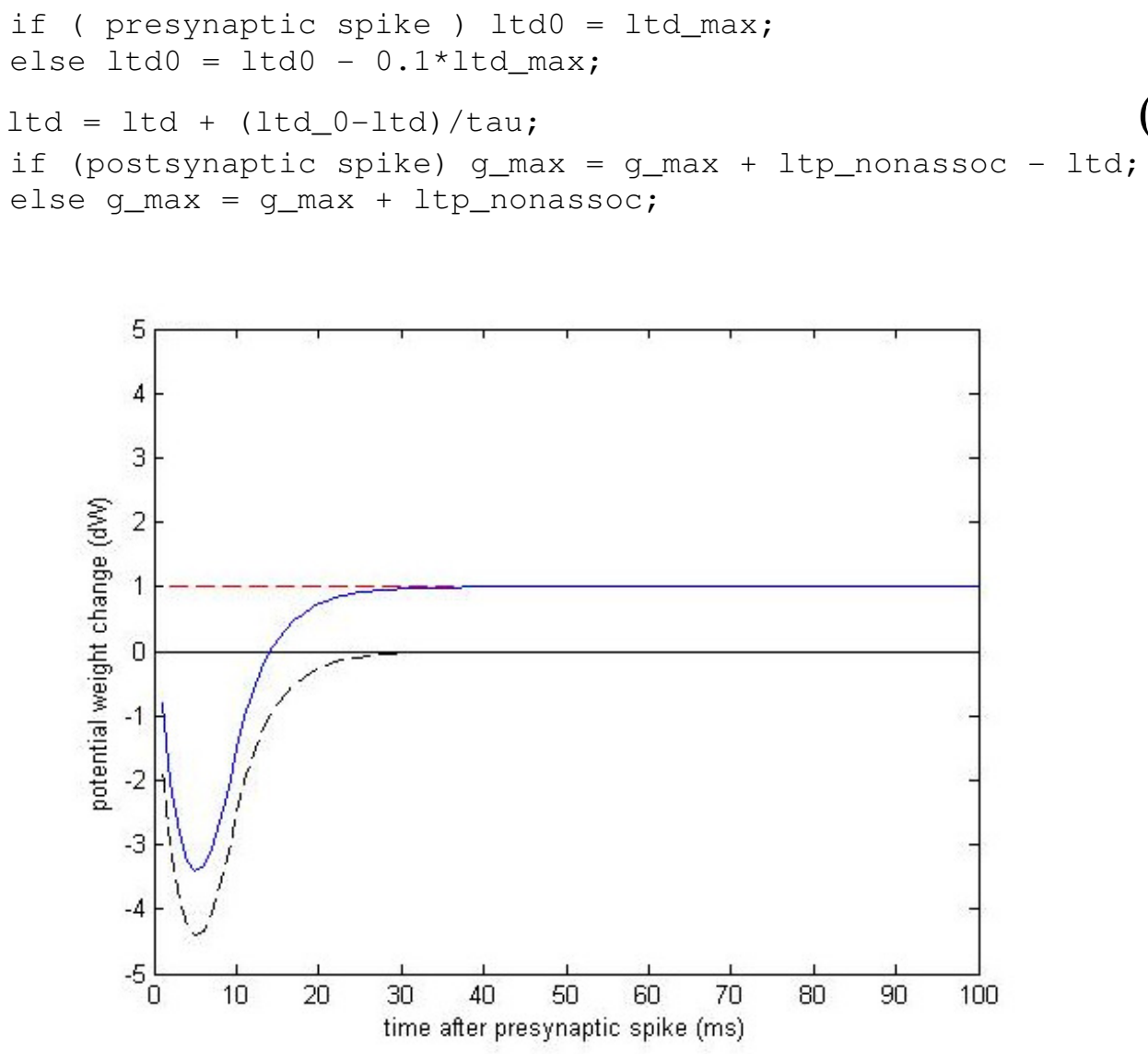

Fig. 5.9: Model of synaptic plasticity 
This rule is applied to each of the EOCD inputs described in the previous section. The cells the synapse at the parallel fiber put out 100 presynaptic spikes throughout the run with $1 \mathrm{~ms}$ spacing, making it so at every ms one cell has a maximum ltd0, and then decays back down to 0 in the following time steps. If there is no post-synaptic spike, the cells' weights increase due to the nonassociative potentiation. If there is a postsynaptic spike, then the calculated ltd is subtracted from the maximum conductance, and reduces the synaptic weight of cells with high ltp values.

Over multiple runs, this process results with a stabilized set of weights, shown in Fig. 5.10. These weights are used to compute the EOCD input from the parallel fiber by multiplying each input with its respective weight. This produces a pattern shown in Fig. 5.11, which is the EOCD input of Fig. 5.8 multiplied by the synaptic weights on 5.10. The set of weights continue to fluctuate slightly throughout the simulation, as the nonassociative potentiation continues to be added if there was no post-synaptic spike. The positive change resulting from this nonassociative potentiation makes it more likely that a post-synaptic spike will occur in the next run, which then brings the weights back down. This is the process that leads to a stabilized set of weights. 


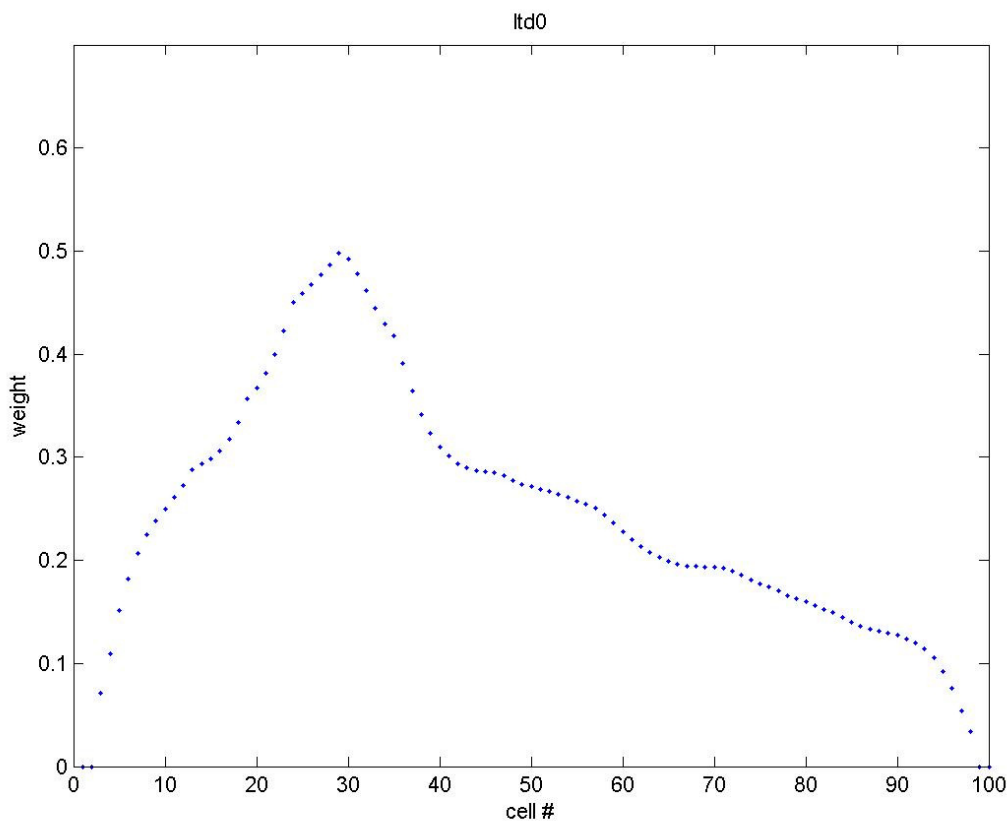

Fig. 5.10: Weights after synaptic plasticity

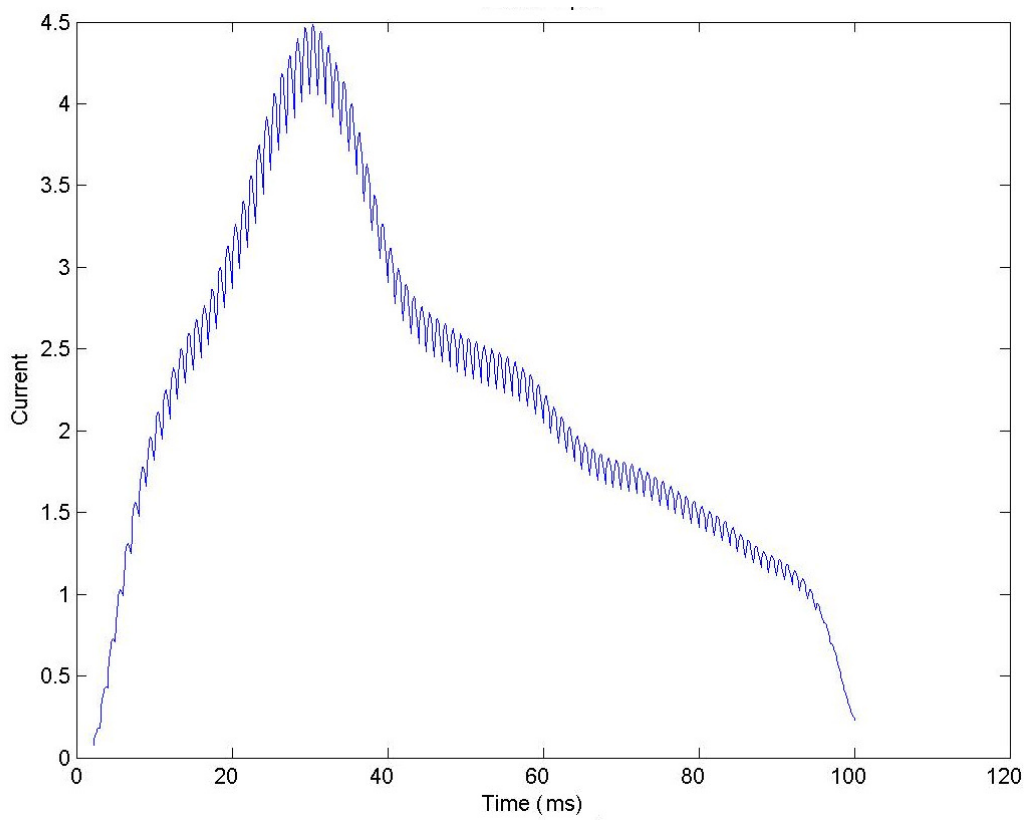

Fig. 5.11: EOCD input after weights are multiplied

\subsubsection{Broad spikes}

As mentioned in the previous chapter, section $4.2, \mathrm{MG}$ cells have been shown to display two types of spike patterns. Narrow spikes originate in the axon and have a lower 
threshold, while broad spikes originate in the dendrites and have a higher threshold. The narrows spikes carry information to the next cell, and broad spikes trigger associative depression of the parallel fiber synapses (Roberts 2000). Due to their importance as the triggers of spike-timing dependent plasticity, broad spikes were modeled and incorporated into the model. Their spike times were recorded and used to find the relative timing of pre- and post- synaptic spikes, which then determined the synaptic weight change.

\subsubsection{Proprioception}

Tail position's affect on cellular activity was discussed in section 3.2.1: Across the brain, body, and environment. Sawtell and Williams demonstrated that there are granule cells that project to the ELL through parallel fiber inputs, which have different firing rates at different tail positions, shown in Fig. 5.12 (c2). Proprioceptive signals are a major input to granule cells, which regulate the firing patterns sent to the ELL. Fig. 5.12 (c2) shows a particular cell's response pattern to different tail positions, with EPSP rates increased as the tail approaches one side. There might be multiple such cells that have different response patterns to proprioceptive input. These proprioceptive inputs are hypothesized remove the electrosensory consequences of tail bending, by using anti-Hebbian STDP at the parallel fibers inputs. This plasticity learns to predict stimuli by forming a negative image and by doing so remove the effect of tail bending (Sawtell 2008, 2010).

This was implemented in the computational model by using proprioceptive input in the range of -1 to 1 , with -1 indicating the tail is all the way on the contralateral side and 1 indicating the tail is on the ipsilateral side. This value controls the probability of 
firing of two different cell types, as proprioceptive input controls firing rates in the real ELL (as shown in Fig. 5.12 (c2)). A third set of cells used in the model does not respond to proprioceptive input. The two cellular sources of proprioceptive input spike maximally when the tail is all the way on one side or the other, as demonstrated in the simulated data shown in Fig. 5.12. This diagram plots the two different cells' firing pattern at two different tail positions: $15^{\circ}$ and $30^{\circ}$. (a1) shows a cell that is firing maximally at the tail position of $30^{\circ}$, the same cell is shown in (b1) firing less than maximally. The second cell does not fire at all when the tail as at these positions, but it would fire if the tail was on the other side of the body.

The final version of the model used in this thesis used slightly different inputs than the ones discussed above and diagrammed in Fig. 5.12. These inputs were shifted slightly off of zero activation when the tail is at position 0 . A more accurate activity patterns was used that better reflects the inputs measured in the ELL, which had the inputs' point of zero activity shifted slightly off of central position. This has both proprioceptive inputs slightly activity when the tail is in the center position.

These different cells provide input to the MG cells at every time step, and synaptic plasticity works on them to adjust the weights towards a stable point, as described in the section on synaptic plasticity. When the tail is at one position a certain set of cells spike pre-synaptically, and this causes plasticity to change the weights towards a stable point for this particular set of inputs, which removes predictable input given the position of the tail. When the tail moves elsewhere, a different set of cells is activated and trained upon, removing predictable stimuli. 
The two inputs of proprioceptive information discussed in this section are hypothesized in this thesis to be the relevant proprioceptive inputs which act to oppose the sensory consequences of tail position. The model's simulation will shed light on the truth of this hypothesis by either removing the sensory consequences of tail movement or not removing them. If the results are negative, it might be due to an insufficient amount of proprioceptive inputs upon which STDP can train to produce adaptive filters.

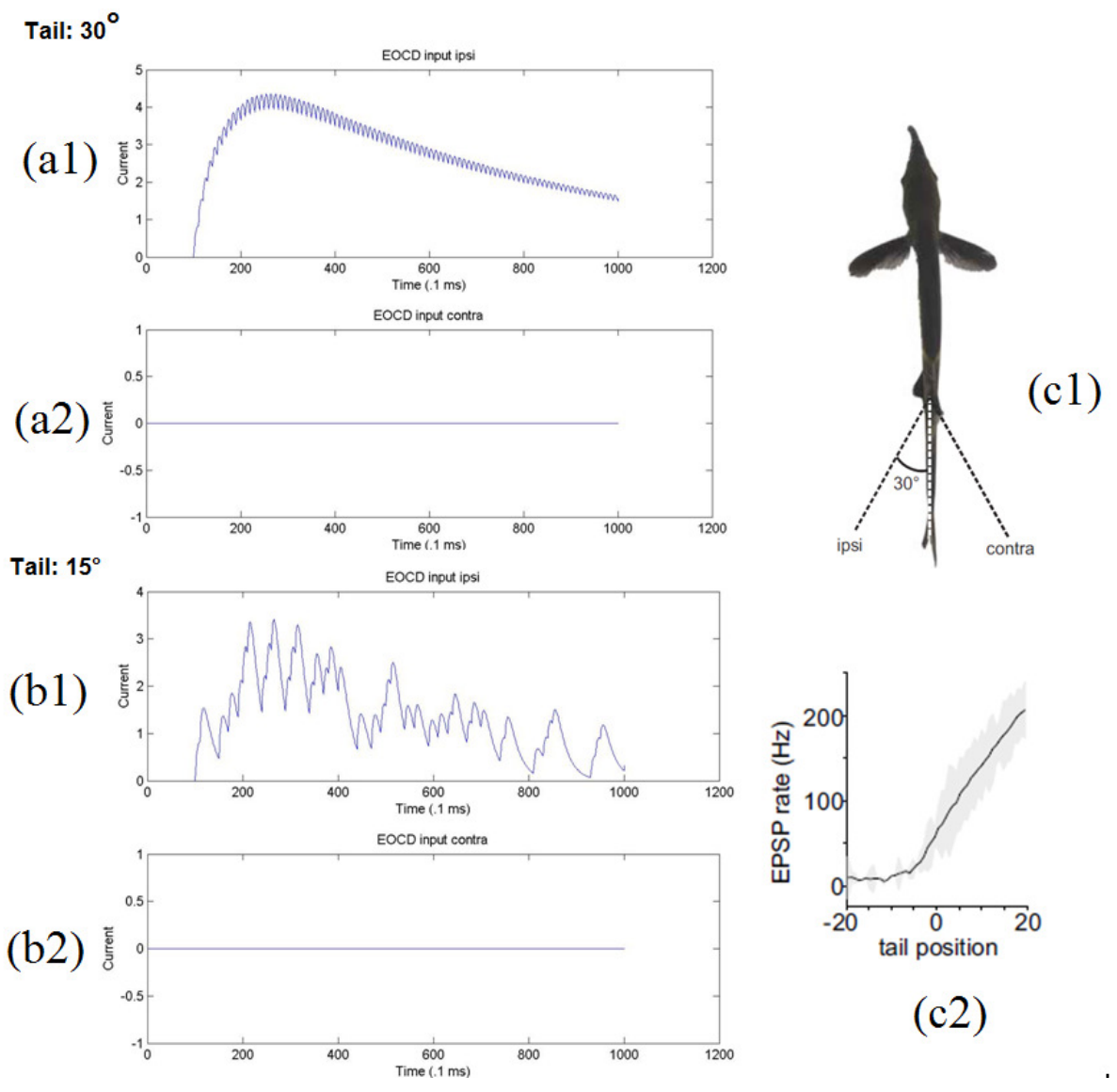

Fig. 5.12: Proprioceptive input

\subsubsection{Body motion effects}

When the tail is moved, it is not sufficient to just simulate proprioceptive inputs in a model. It is also necessary to include the effect of this body position on the pattern of sensory stimuli provided to the ELL. When the tail is turned to one side it increases the 
electrical current on that side of the body, and enhances the strength stimuli have on the electroreceptors.

In order to incorporate this in the model, a short line of code was written which set a tail position between the values of -1 and 1 (from the contralateral side to the ipsilateral side). This value is multiplied by .2 and added to the original stimulus before tail movement was accounted for. This made it so that if the tail is on the ipsilateral side, it has a positive value (between 0 and 1) which is multiplied by .2 and added to the stimulus, therefore increasing the stimulus' amplitude. If the tail is on the contralateral side the tail position is negative, and reduces the stimulus' amplitude. These values were made up for this thesis, and are just guesses at what change that occurs in reality.

This increase in stimulus amplitude due to tail movement leads to changes in the electrical propagation through the ELL. When it makes it to the MG cells, it is combined with the parallel fiber input from proprioceptive areas to determine the MG spike pattern. These parallel fibers undergo plasticity to remove the increased field of electrosensory stimuli. Therefore when the tail is moved, the appropriate parallel fiber inputs are activated and remove the effect of the tail position from the sensory stream.

\subsubsection{Efferent cells}

Efferent cells connect to MG cells and granular cells (see Fig 5.2). Their activity has been recorded, and is shown in Fig 5.13. For this thesis, efferent cell activity was modeled with a $2 \mathrm{~d}$-IF model and connected to its respective granular and MG cells. The parameters used to generate the model are listed in Table IV, and the resulting activity is shown in Fig 5.14. Two types of efferent cells were included: Large Ganglion (LG) and 
Large Fusiform (LF). These cells differ only in their connectivity, LF cells receive excitatory input from granular cells, and inhibitory input from MG1 cells. LG cells receive inhibitory input from granular cells and inhibitory input from MG2 cells. This connectivity was implemented using the same approaches previously described, by converting input cells' voltage into current.

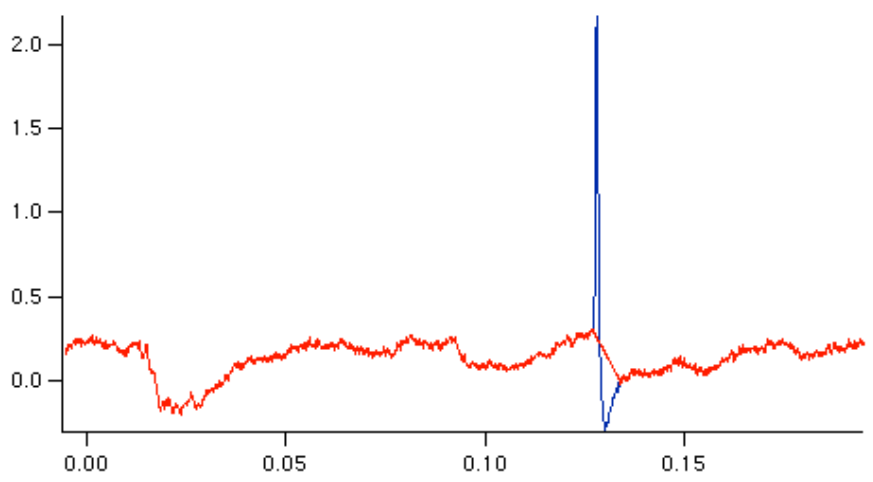

Fig. 5.13: Efferent cell activity (Mohr, Roberts, Bell 2003)

Table IV: Parameters for efferent cells

\begin{tabular}{|c|c|c|c|c|c|c|c|c|c|}
\hline $\mathrm{a}$ & $\mathrm{b}$ & $\mathrm{c}$ & $\mathrm{d}$ & V_el & V_r & v_t & C & k & V_peak \\
\hline 5 & -2 & -60 & 500 & 0 & -60 & -40 & 50 & 10 & 10 \\
\hline
\end{tabular}

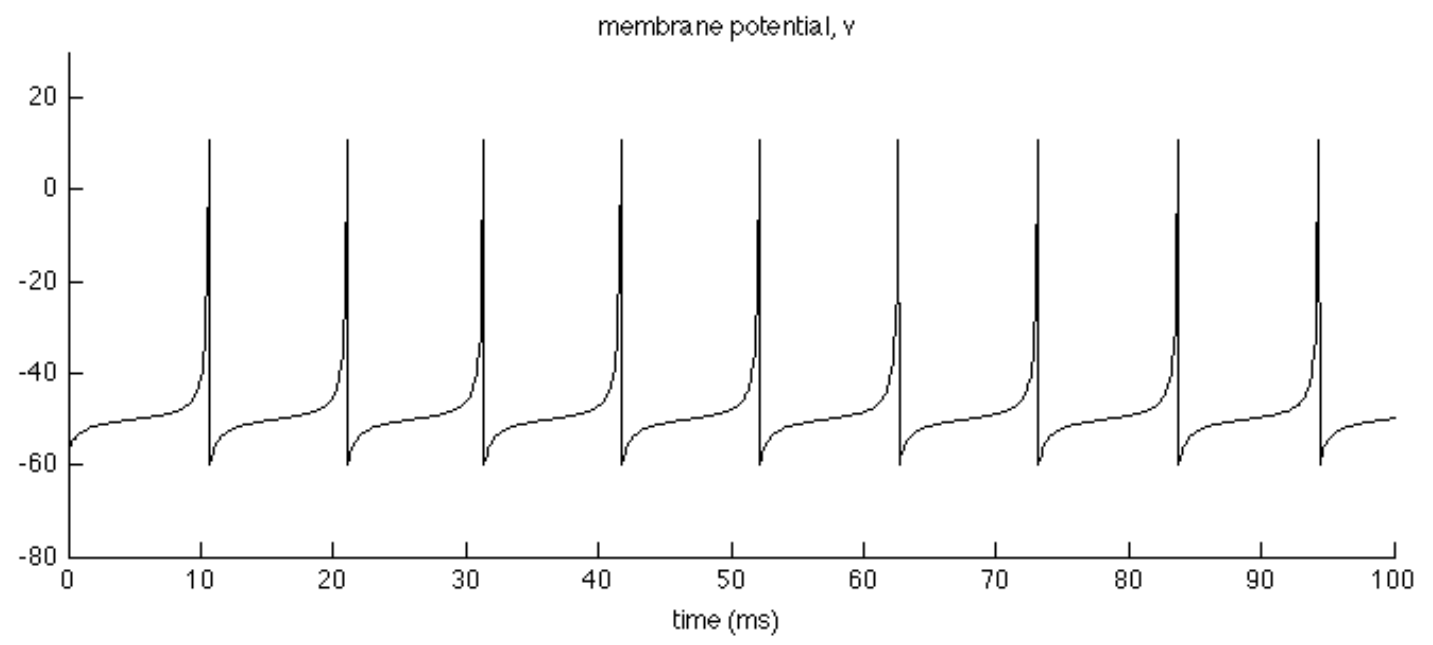

Fig. 5.14: Efferent cell simulation with the 2d-IF parameters of Table IV 


\section{Chapter 6}

\section{Results}

Simulations generate data of predicted ELL function based on the programmed rules of the model. This data needs to be compared with data obtained from real ELLs to provide conclusions about the sufficiency and success of the model in reproducing ELL function. To accomplish this task, this thesis uses visual comparisons as the primary method of interpreting results; by generating visualizations of the simulation data under different conditions and visually comparing them with graphs of neurophysiological data, the similarities and differences are discovered. Comparisons described in this chapter include simulated MG cell activity, efferent cell activity, and the adaptive filter function's ability to remove the sensory consequences of body position by training on proprioceptive input. The successes of the model are discussed as well as its limitations.

If the model is deemed sufficient in describing a function of interest, it can be used to test hypotheses regarding that function. There are multiple current hypotheses regarding the ELL that are difficult to test through biological means. This chapter examines a couple of these possibilities through testing different parameters. These include a test about excitatory electrical synapses between granular cells and MG2 cells as well as granular and LF cells. A second test regarding the sufficiency of MG cell plasticity to produce a plasticity-like effect in efferent cells is also produced. Simulated evidence provides motivation for further biological experimentation and drives the development of scientific knowledge. 


\subsection{MG cell activity}

MG cells play a significant role in the functioning of the ELL as the primary cells of the cerebellum-like structure, and the most plastic of the ELL's cells. This makes the understanding of their function an important scientific endeavor for the understanding electrosensory processing in mormyrids. Their simulated activity was recorded under multiple conditions to examine the underlying patterns of their activity in the ELL.

In this section, MG cells are examined in baseline conditions: without additional simulated tail movements or other such scenarios. The model was simulated across many runs to examine the effects of a stimulus on the MG activity. The simulation begins with no stimulus, after multiple cycles a stimulus was injected for a prolonged period, and finally the stimulus was removed and the model continued running without a stimulus. This sequence allows us to examine how the MG cells operate at baseline before a stimulus, how the MGs respond to the onset of stimuli, how they adapt throughout a stimulus' injection, and how they respond when the stimulus is removed.

Raster plots of this scenario were created, and shown in Fig. 6.1, which plots MG1's spike times, and Fig. 6.2, which plots MG2's spike times. In both of these graphs the early runs are shown on the top of the graph and later runs are at the bottom. Throughout the simulation a stimulus is turned on at run 150 and turned off at run 350 , and the model continues to run until run 850 , as marked on the right side of the graphs. These raster plots display the two different spike types: broad spikes in magenta and narrow spikes in blue (shown in the key). As mentioned in section 4.2, narrow spikes originate in the axon and have a lower threshold; broad spikes originate in the dendrites 
and have a higher threshold. Narrows spikes carry information to the next cells while broad spikes trigger associative depression at the parallel fiber synapses.

In Fig. 6.1, MG1 cells begin the simulation without electrosensory input. At run 150 a stimulus is injected. Electrosensory input is inhibitory to MG1 cells and so it inhibits the broad spikes that were previously firing around the $30^{\text {th }}$ millisecond of each run. Due to the removal of these broad spikes, non-associative plasticity drives the synaptic weights up, which shifts narrow spike times earlier in each run. When the stimulus turns off, the broad spikes are no longer inhibited, and so they begin firing early due to the highly increased synaptic weights that formed throughout the stimulus. The broad spikes drive the weights back down due to the associative anti-Hebbian STDP. This can be seen by the curve of broad spikes that begin earlier when the stimulus turns off, and are pushed to later times in the run.

Fig. 6.2, of MG2 cell activity demonstrates how a stimulus' onset excites the cell and makes broad spikes occur earlier on in the run. The stimulus' offset removes the excitation, and with less excitation broad spikes no longer occur early in the cycle. This turns off the associative plasticity and allows nonassociative plasticity to increase conductance of earlier currents, which create narrow spikes that approached earlier and earlier in the cycle.

Similar behavior can be seen in the graphs of electrophysiological data shown in Fig. 6.3 of MG1 activity and Fig. 6.4 of MG2 activity. These plots are arranged in the same way as the simulated plots; with early runs on top and later ones on the bottom. Narrow spikes are black points and broad spikes are red points. Fig. 6.3, of MG1 activity, has a stimulus injected around run 250, and removed around run 510. Just as in 
the simulation, the electrosensory input inhibits MG1 cells, which remove much of the broad spike activity. But unlike the simulation, in this case the inhibition pushes the narrow spikes later in the cycle. When the stimulus is removed, the same curve of broad spikes is demonstrated as in the simulation. This is due for the same reasons as the simulation: the removal of stimulus increases the input current, triggers broad spikes and leads to associative plasticity that drives synaptic weights down. This makes the broad spikes occur later in following cycles. The MG2 activity of Fig. 6.4 also demonstrates the same features of the simulation: when the stimulus turns on, excitatory input drives the broad spikes to occur early on in the cycle (they did not even occur previously). When the stimulus is removed, the broad spikes disappear and nonassociative plasticity increases synaptic weights. This makes the narrow spikes appear earlier on in the following cycles, though not as noticeably as in the simulation.

There are apparent differences between the simulated data and the electrophysiological data: the relative spike times throughout the runs are different, the rates of adaptation are different. The cell recorded in Fig. 6.3 appears to have much more noise than the simulated model. This noise is seen by the spreading narrow spikes. Other differences that can cause these differences might be the relative strengths of the inputs, or the parameters controlling the spike shapes are likely a little different in the model. Overall the same patterns exist, which indicate the sufficiency of the model's structure and plasticity in capturing the underlying mechanisms of the ELL's function. 


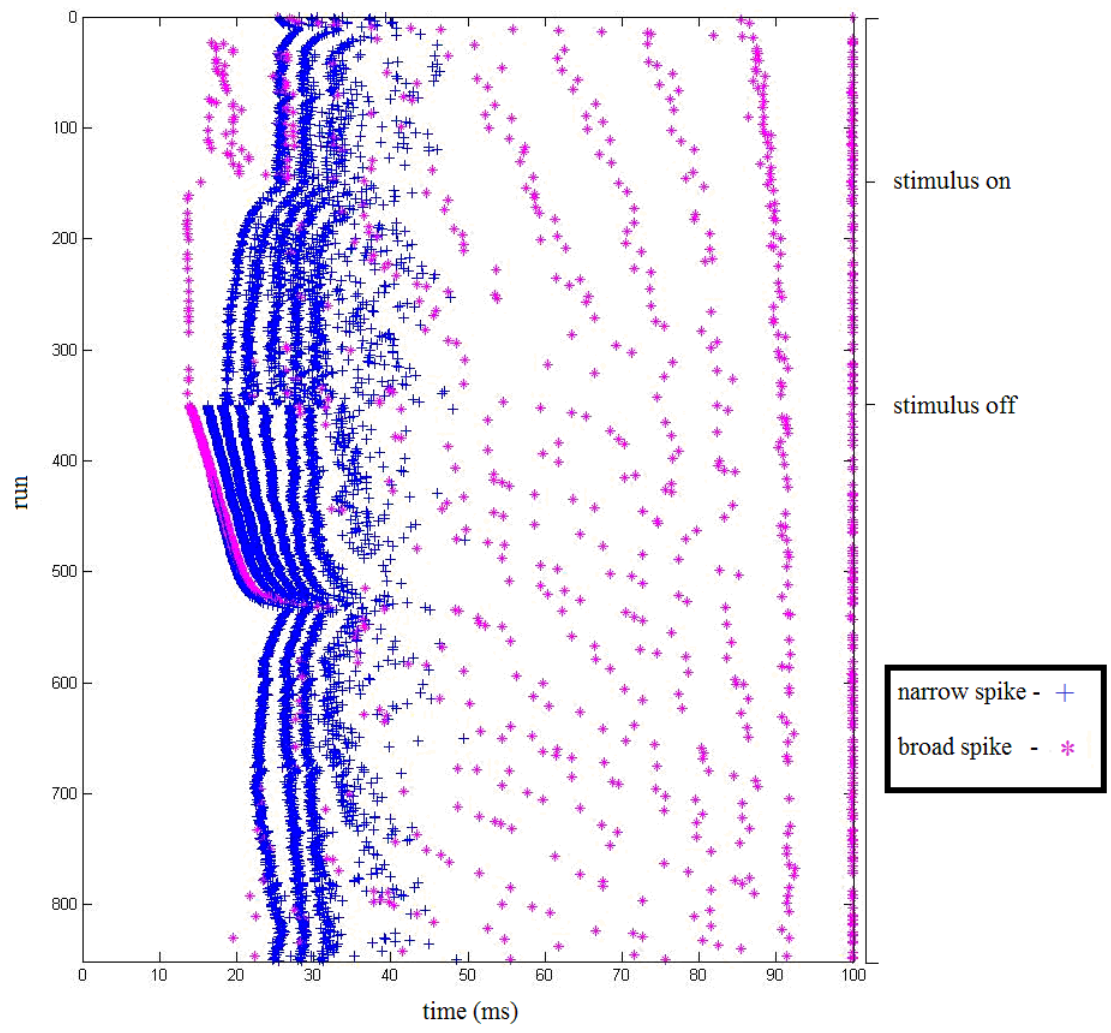

Fig. 6.1: MG1 raster plot, simulation

Narrow spikes are blue, broad spikes are magenta. Stimulus comes on at run 150, and turns off at run 350

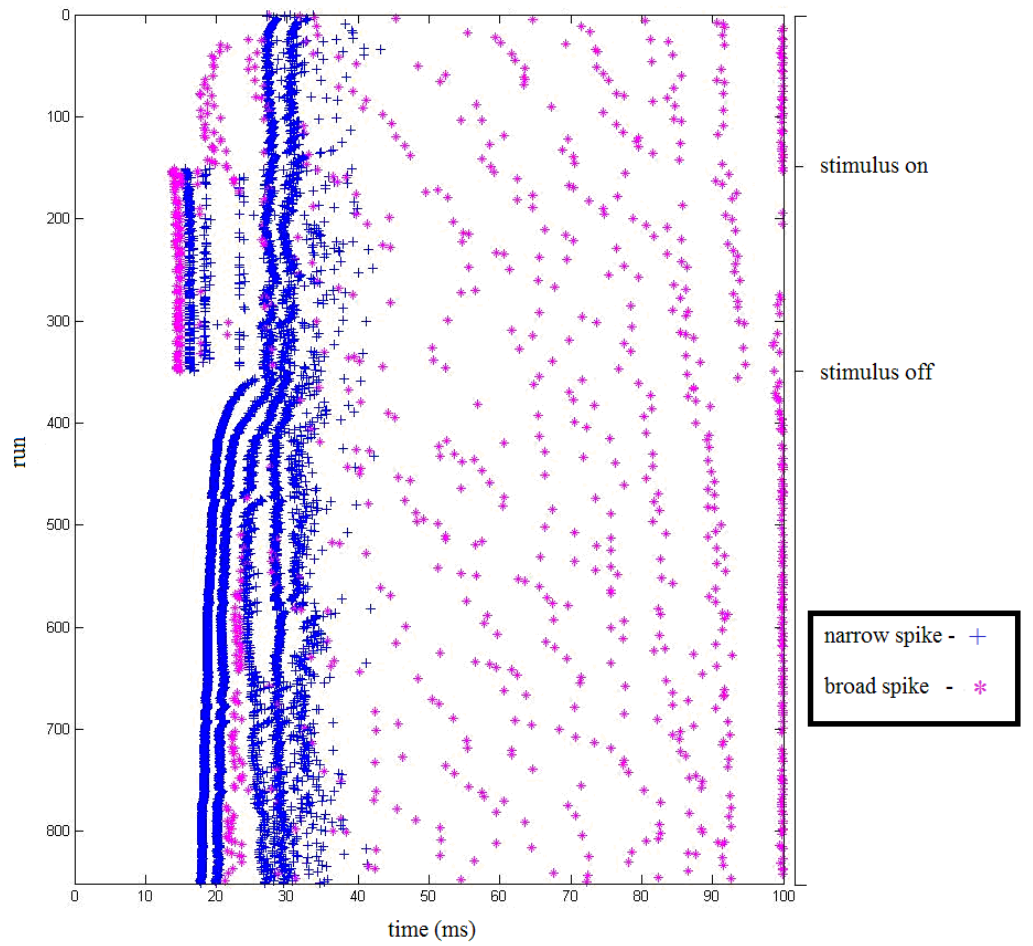

Fig. 6.2: MG2 raster plot, simulation

Narrow spikes are blue, broad spikes are magenta. Stimulus comes on at run 150, and turns off at run 350 


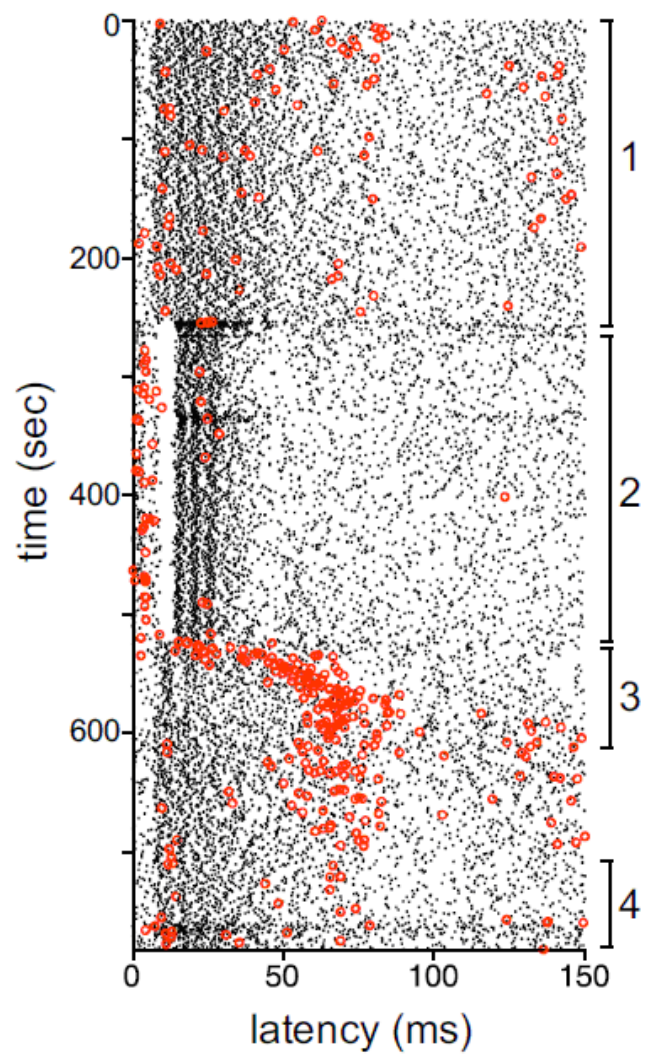

Fig. 6.3: MG1 raster plot, electrophysiological data (Sawtell 2007)

Narrow spikes are black, broad spikes are red. Stimulus comes on at run 250, and turns off at run 510

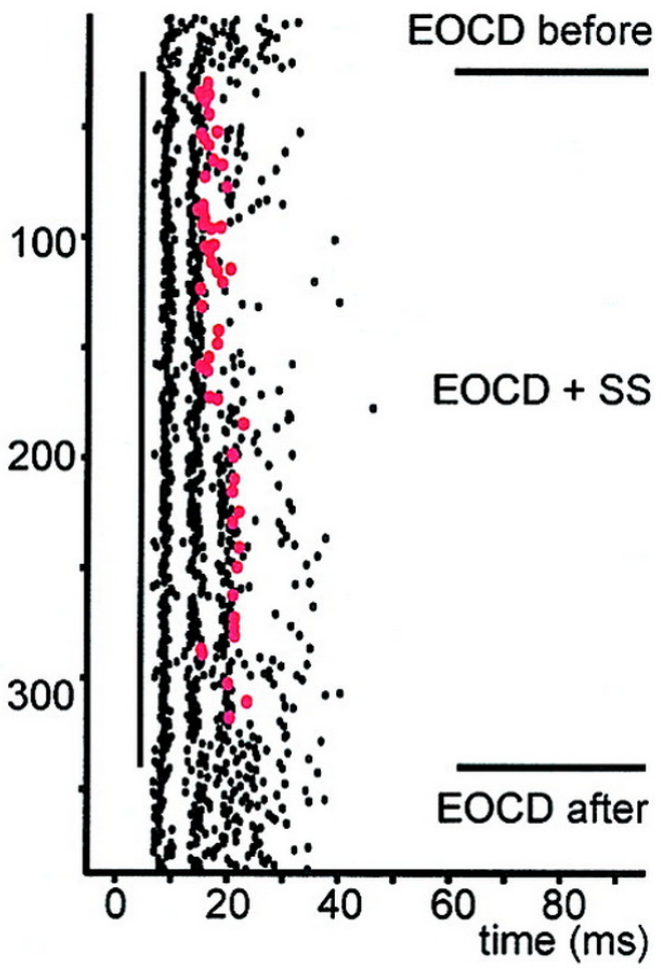

Fig. 6.4: MG2 raster plot, electrophysiological data (Mohr 2003)

Narrow spikes are black, broad spikes are red. Stimulus comes on at run 25, and turns off at run 325 


\subsection{Efferent cell activity}

Efferent cell activity was recorded in a similar scenario as the MGs of the previous section: a stimulus is turned on partially through the simulation, is run for a period of time, and is then turned off. The visualization of this simulation is approached in a different manner due to the limited available diagrams of electrophysiological data that can be used for comparison. LF and LG cells have their activity recorded across a single run before the stimulus, during the stimulus, shortly after the stimulus is removed, and long after the stimulus is removed. Results of this simulation are shown in Fig. 6.5. This simulation data can be compared with the electrophysiological data of a Fig. 6.6.

Comparison of these results demonstrates the similarity of the model to reality: LF cells have low activity before stimulus, high activity during stimulus (due to their excitation by electrosensory input), and reduced activity following the stimulus's removal. Fig. 6.6 shows noisy LF cells due to the approach that was used for recording, but the same general pattern can be seen with reduced activity before stimulus, increased activity during stimulus, and then even further reduced activity when the stimulus is removed. Simulated LG activity is also similar to the electrophysiological data. The stimulus inhibits activity due to inhibitory connections between granular cells and LG cells. When the stimulus is removed so is the inhibition on LG cells, this results in increased activity. In the simulation this increased activity (shown in Fig. 6.5 in the LG cell's $3^{\text {rd }}$ plot titled "LG after") took longer than expected to occur, the shown plot was generated several runs after inhibition was removed. This delay occurred due to unknown reasons not predicted by the theory. Possible reasons for this difference are discussed in the discussion section of this chapter, section 6.5. 

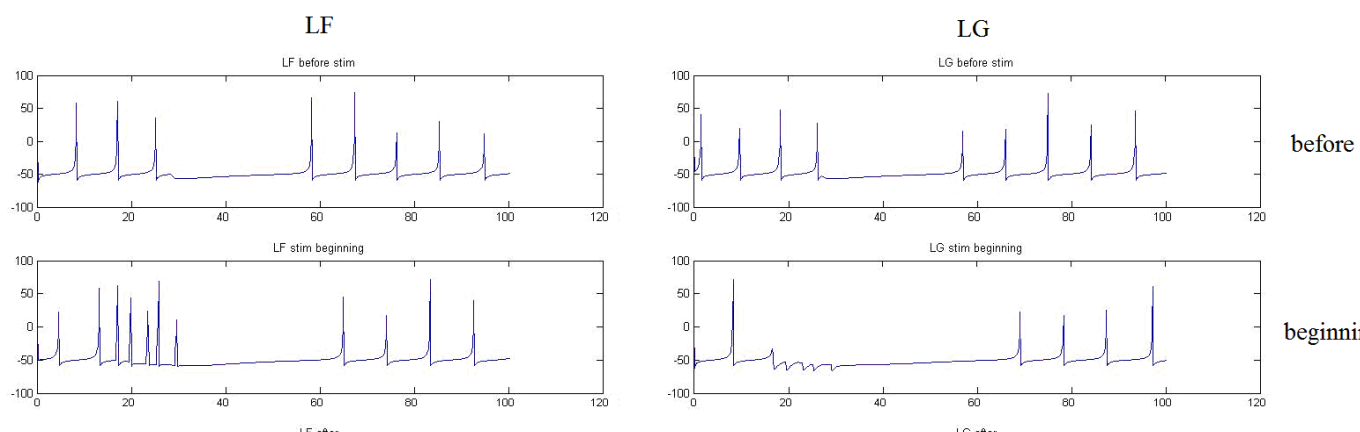

beginning stim
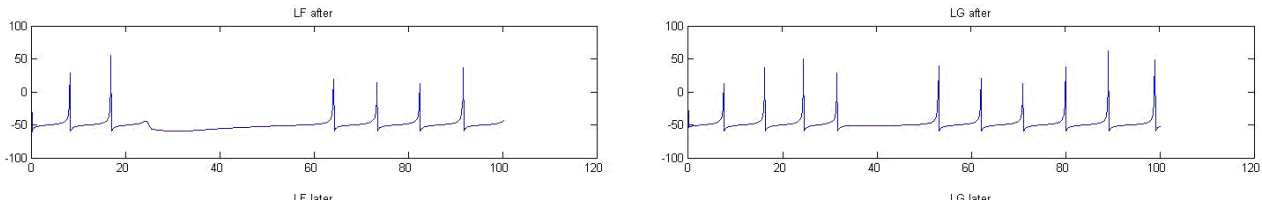

after stim
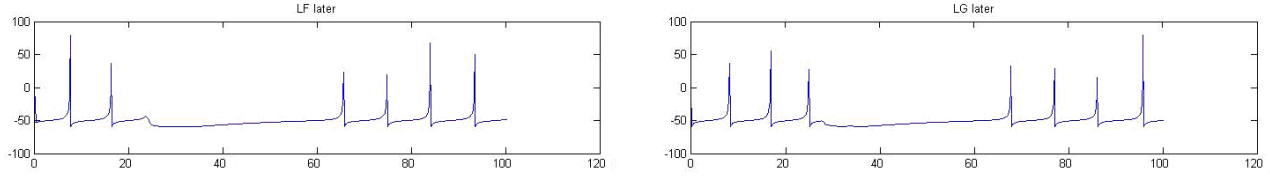

long after

Fig. 6.5: Simulated efferent activity

LF activity is on the left, LG activity is on the right. The top plots of both cells were taken before a stimulus injection. The second was at the beginning of a stimulus. The third is a little after the stimulus was removed. And the bottom plot was taken far after the stimulus was removed.

\section{Large fusiform cells Large ganglion cell}
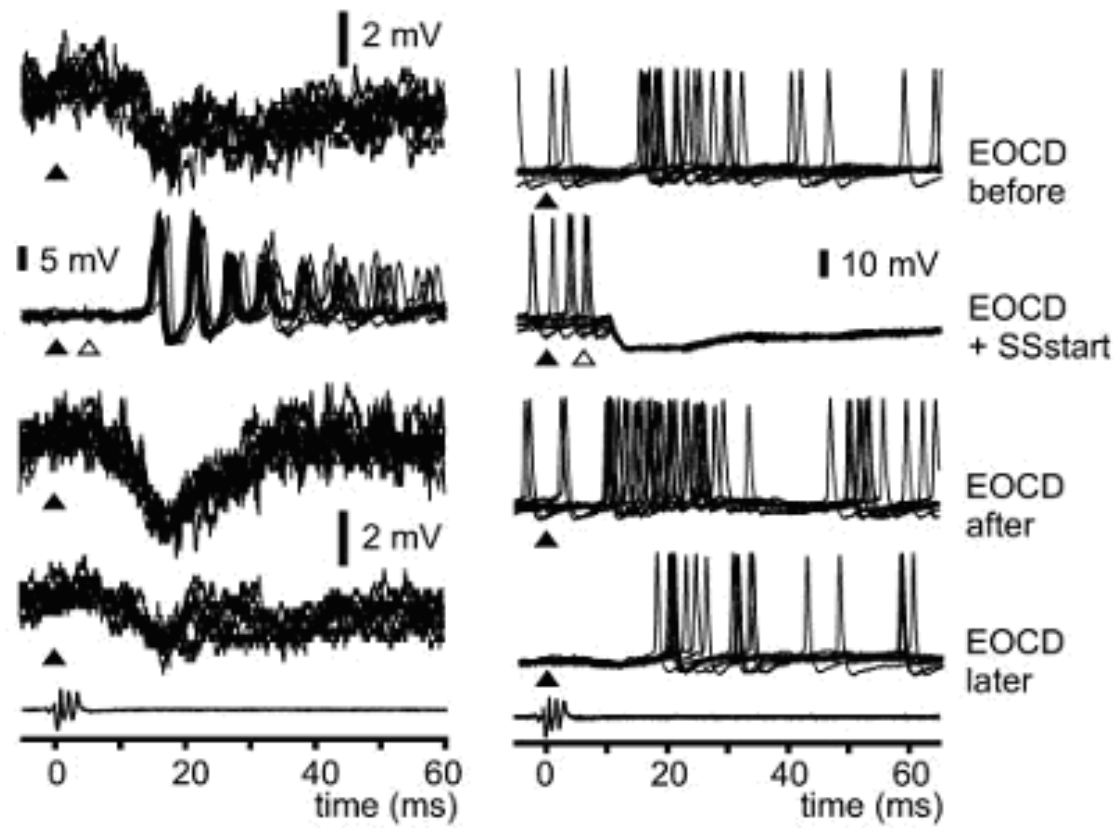

EOCD

after

Fig. 6.6: Efferent activity (Mohr 2003) 


\subsection{Removing the sensory consequences of tail movement}

The main goal of this thesis was to build a model that could demonstrate how the ELL removes the effects of body movement from its sensory stream. It was hypothesized that proprioceptive inputs, which have been observed in the ELL, adapt through the MG cell's STDP to remove the sensory consequences of movement. To test this through simulation, two sources of proprioceptive input were incorporated in the model to provide MG cells with information regarding the tail's position. Fig. 6.7, previously illustrates the how two proprioceptive inputs behave. For the input diagrammed second from the left, when the tail is a little off center position ( around tail position -5) there is no proprioceptive inputs. As the tail moves towards the ipsilateral side (tail position 20) the input's activity increases. The second input is the exact mirrored opposite.
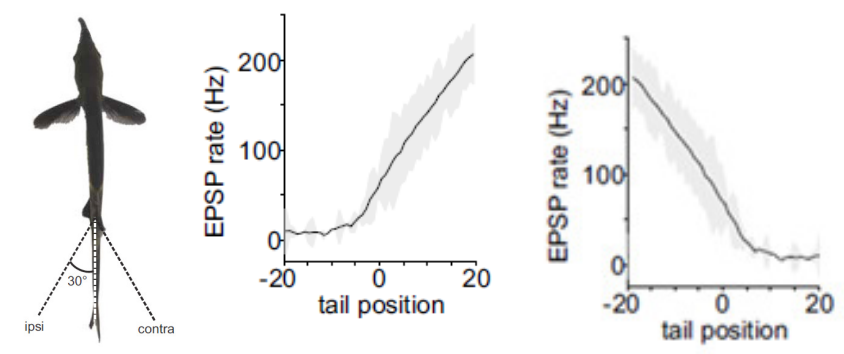

Fig. 6.7: Tail position's effect on cellular activity (Sawtell \& Williams 2008, Sawtell 2010)

The model was simulated with these mechanisms to test if it is able to remove the sensory consequences of tail movement after a long training period in which the synaptic weights can adapt to the proprioceptive inputs. To accomplish this, a simulation was created that had three main stages: a short pre-training run, a long training run, and a short post-training run. Throughout each run the tail was moved from the ipsilateral side, to the center position, to the contralateral side. This simulation allows us to visualize 
how the ELL behaves before training by looking at the pre-training session, and then compare it to the post-training session to see how the training session changed the activity. The difference between these two sessions would be due to the adaptation that takes place through the long training session. The hypothesis posed by this thesis predicts that the pre-training session should have apparent differences when the tail is move, and the post-training session should have the consequences of movement removed.

The results are show in Fig. 6.8 with the MG cell responses during the pretraining session and Fig. 6.9 with the MG cell responses during the post-training session. Fig 6.10 shows efferent responses during pre-training, and Fig. 6.11 shows efferent responses during post-training. As written in the diagrams, the sessions are divided into a period in which the tail is held all the way ipsilaterally, and then moved to the center, and then moved contralaterally.

Both of these cells' activity appears to have adapted over the training session, though they do not produce a complete adaptive filter. By training on the proprioceptive input, the MG cells were able to adjust their synaptic weights and make it so that the tail positions had less of an effect on activity. This is demonstrated by the cells' activity during the post-training session being less different from each other when the tail is moved than in the pre-training session. MG1 cells generate activity during center position that was absent in the pre-training session, and the ipsilateral and contralateral activity appear more similar. MG2 cells have ipsilateral activity shifted later in the cycle and contralateral activity shifted earlier. Efferent activity also changes slightly, with the contralateral activity shifting earlier. These results demonstrate that there is a partial 
adaptive filter in place. However, a complete adaptive filter would make the responses at different tail positions exactly the same after training.
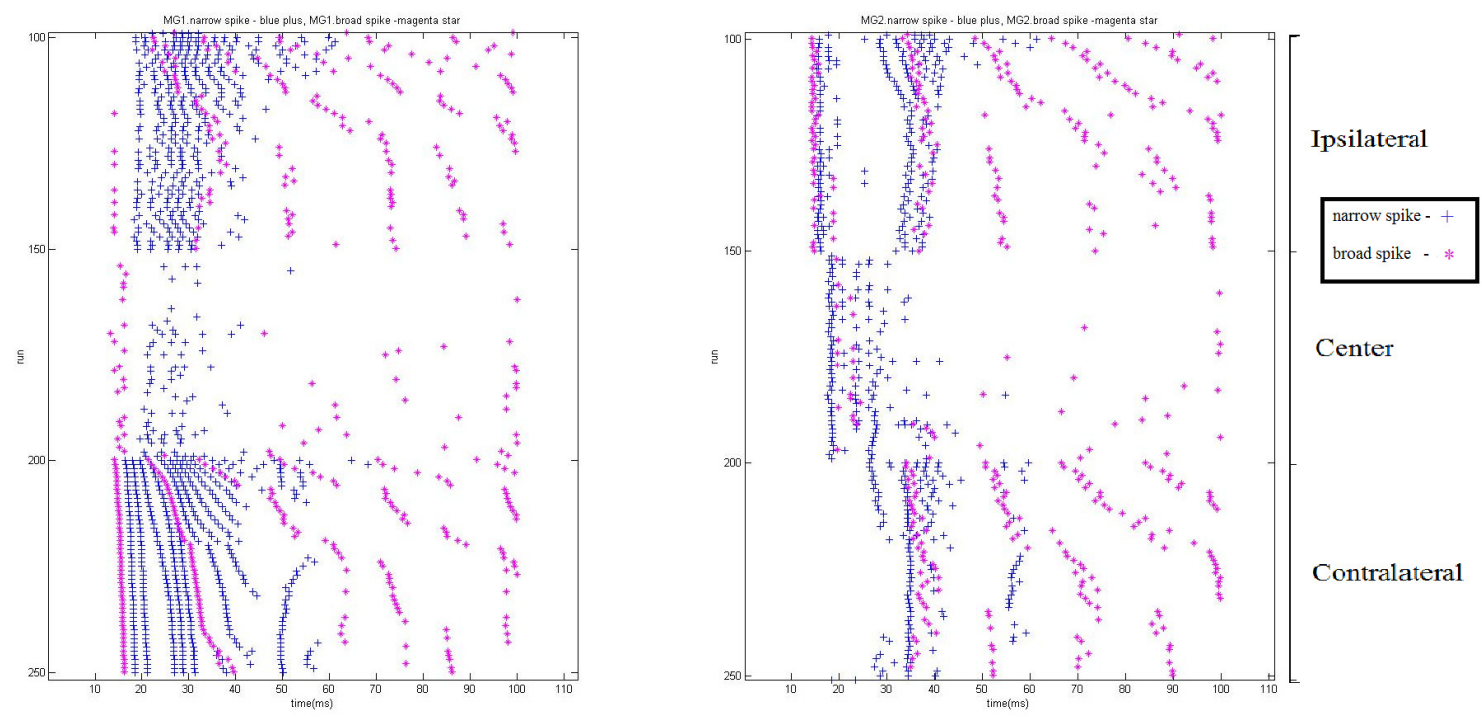

Fig. 6.8: MG1 (left) \& MG2 (right) pre-training
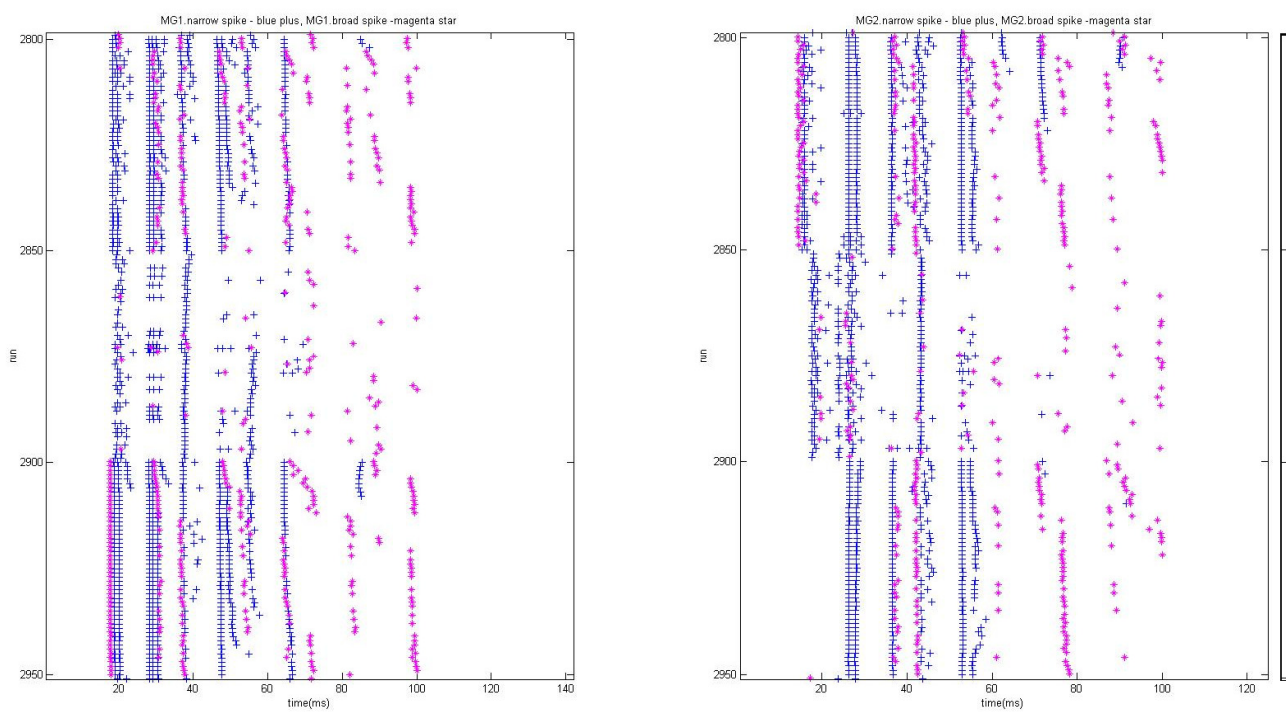

Ipsilateral

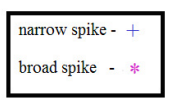

Center

Contralateral

Fig. 6.9: MG1 (left) \& MG2 (right) post-training 


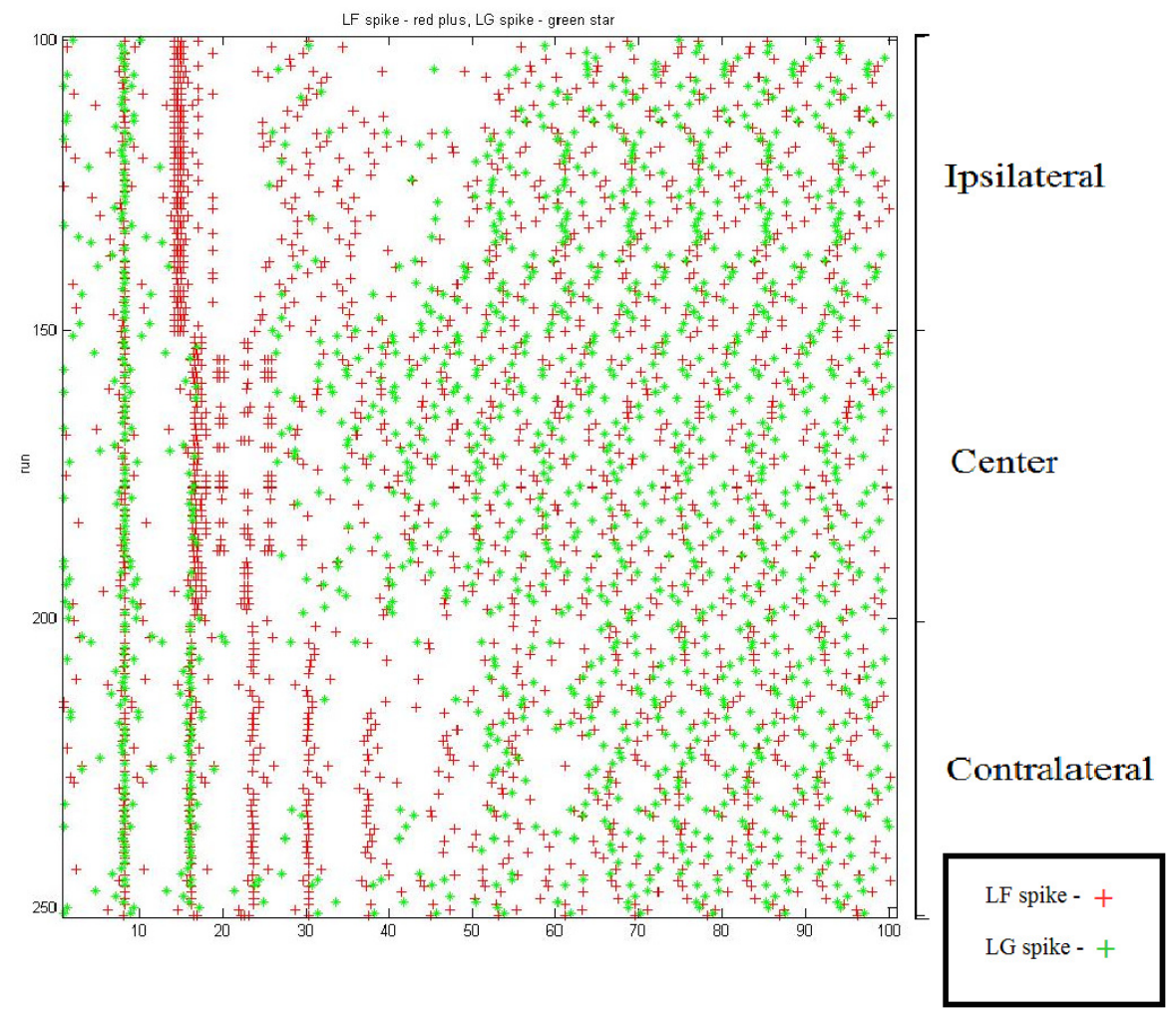

Fig. 6.10: Efferents pre-training

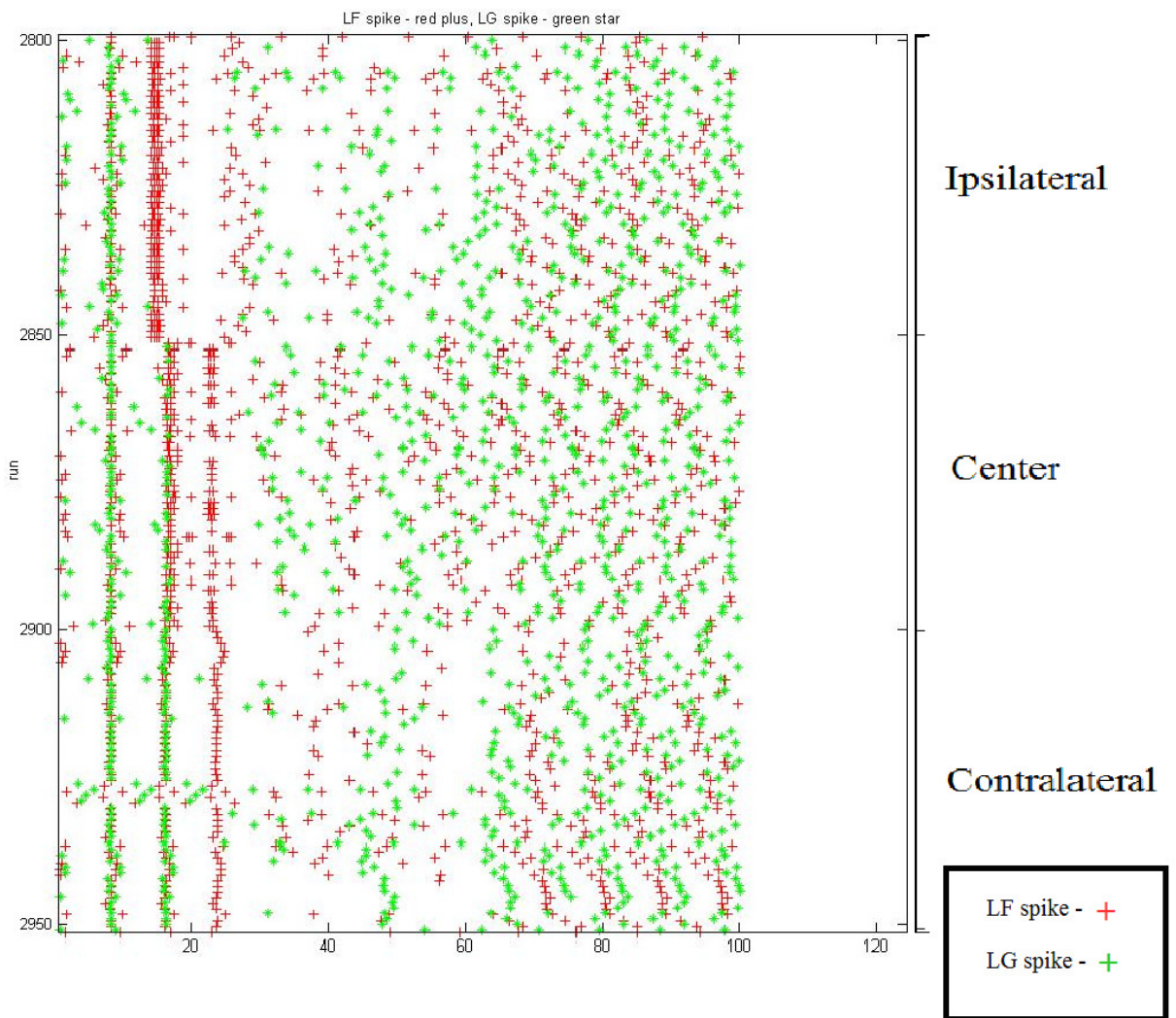

Fig. 6.11: Efferents post-training 


\subsection{Testing hypotheses}

Having a computational model does not only provide a way to confirm our structural knowledge of a system; it can also provide a means to test hypotheses regarding different components in the system. For this thesis, two tests are run to demonstrate how different possibilities can be tested with the model. The first test involves the apparent plasticity of efferent cells. The second involves the presence of excitatory electrical gap junction between granular cells and MG2, as well as between granular cells and LF cells. Results are discussed as well as their indications on how the ELL really works. More exact hypotheses tests can be done in future work to quantitatively measure the differences and draw more conclusive results.

Real efferent cells display activity that appears plastic. This has led some researchers to hypothesize that the efferent cells are plastic, while other attribute the behavior to MG cell's plasticity producing a plastic-like effect in efferent cells. The ability for MG cell's plasticity to make efferent cells appear plastic was tested in this thesis by including plasticity only in the MG cells and looking to the efferent cells' activity for signs of plasticity. This type of activity can be seen in Fig. 6.5, by the increased activity following the stimulus' removal. This figure was produced with 50 MG cells feeding into each efferent cell. Other simulations with fewer MG cells did not produce the same effect. This result indicates that there is a possibility efferent cells plastic behavior could be shaped by large amounts of MG input.

Excitatory chemical synapses were not found in MG cells, yet there is excitatory behavior that indicates there should be some kind of excitatory connection. This behavior can be explained by the possible presence of excitatory gap junctions in the 
between granular cells and MG2, as well as between granular cells and LF cells. This possibility was tested by changing the current of the mentioned synapses from a gap junction-like current to a chemical synapse-like current. These results showed that the same pattern of responses is generated with a gap junction as with a chemical synapse. This indicates that gap junctions and chemical synapses are equally likely to exist in the ELL. Gap junctions are therefore sufficient to explain the excitatory properties of the MG cells, without a need to invent a chemical excitatory synapses that has not been found.

\subsection{Discussion}

The comparison of simulation results with data from real ELL has provided much insight to the sufficiency and success of the model. Some simulations produced results that confirm the sufficiency of the model to reproduce a functional pattern of activity, while other simulations were not successful and point to deficits in the model. While MG results reproduce electrophysiological data nicely, efferent cells only reproduce the general pattern of activity but lack some important tendencies of efferent activity. The proposed cerebellum-like structure, inputs, and MG cell STDP is partially confirmed to be sufficient for the production of an adaptive filter.

The MG cells' simulation is largely successful at reproducing the behavior of real MG cells except for some differences that are likely due to the relative strengths of inputs and the amount of noise in the system. The model also successfully reproduces the general excited or inhibited activity of LF and LG cells in response to a stimulus's onset or offset. But the timing of the spikes and the time taken to produce the bursts in the LG 
cells are far from accurate. This is possibly due to the timing of the stimulus' response in MG cells, which in turn affects efferent activity. Mohr (2003) demonstrates that the timing of electrosensory responses is later and more variable in MG2 cells than the other cells of this thesis' model. This difference in response was not produced by the model, and could reveal some missing components that should be included in the model. If MG2 cells responded later to electrosensory stimuli, then it would likely improve the adaptive behavior of MG2 and LG cells, and could fix the problem in the efferent cells.

The effect of proprioceptive input on producing an adaptive filter of the consequences of movement is also demonstrated by this model, though only with partial success. By providing two sources of proprioceptive input and allowing the model to train its synaptic weights with STDP through an extended training session, the model was able to produce a partial adaptive filter. This demonstrates that the proposed mechanism is likely sufficient, but either the implementation is not completely accurate or there might need to be more proprioceptive inputs.

We also looked at how the model can be used to test different hypotheses by manipulating the model and analyzing the results. The MG cell's plasticity was demonstrated to sufficiently generate some plastic-like effects in efferent cells, which validate that plastic-like behavior in real efferent cells could be due to MG cells' plasticity. The gap junction test demonstrated that electric synapses are sufficient to explain the electrosensory responses seen in the ELL. This result shows that there is no need for excitatory neurons in the system. 


\section{Chapter 7}

\section{Concluding Remarks}

This thesis' model of the ELL is sufficient for explaining some patterns observed in the ELL, but is limited in its ability to explain other patterns. The simulation results discussed in the previous chapter can be used to build and develop our understanding of the ELL and to guide future research efforts. This concluding section discusses the positive results as well as negative results and the insights they grant us. The chapter also discusses the sources of limitation, and suggests solutions as to how they can be fixed in future models. It concludes with a summary of the thesis; what was learned about the ELL, what was learned about computational modeling, and other insights that were gained along the way.

\subsection{Contributions}

Despite its limitations, the ELL model created for this thesis and the simulation results it produced contribute significant knowledge to the scientific effort for understanding electrosensation. The most important contribution made is the creation of the computational model itself. This model is an important step towards producing a complete model that captures the ELL's more complex patterns. It lays out the main structural schema of the ELL and provides the primary inputs, though approximate. The cell types are modeled as dynamical system, with 2-dimensional integrate-and-fire neurons. This makes them able to simulate complex neuronal dynamics across time with 
a reduced computational cost. Spike timing dependent plasticity is implemented successfully and allows the ELL to adapt to stimuli over time, producing an adaptive filter.

This thesis adopted the cerebellum-like structure hypothesis, which claims that the cerebellum-like structure within the ELL is sufficient for generating the electrophysiological patterns observed in the ELL's efferent output. Previously this was but a hypothesis without a testable model, but this thesis used computational neuroscience's approach for creating a model and testing the hypothesis. The model's simulated results have provided confirmation of the cerebellum-like structure as a minimal relevant model, and provide a framework with which the hypothesis can be further scrutinized and tested in future studies.

Results also support the sufficiency of anti-Hebbian STDP at the MG cell parallel fibers synapses. This rule was implemented in the model, and successfully shaped the synaptic weights in a way that produced the same electrophysiological patterns seen in the MG and efferent cells of real ELLs. The sufficiency of this plasticity at the MG cells' parallel fiber synapses is demonstrated through the test on efferent cell activity, which indicated that MG cell plasticity can explain the plastic effects that have been observed in efferent cells.

This thesis tested Sawtell \& Williams hypothesis that proprioceptive input provides a signal upon which synaptic plasticity can train and remove the sensory consequences of tail movements. Results confirm this hypothesis and demonstrate that the two included sources of proprioceptive input are sufficient to produce the effect to an incomplete filter (section 6.3). The partial success of the filter does reinforce that the 
adaptive filter function is possible with the basic framework of the model, but requires some additional work to be complete. It is not certain if the incompleteness is due to poor implementation or if additional inputs are required.

With the confirmation of multiple hypotheses and a working computational model, the study of electroreception can move forward in its scientific endeavor. Results provide a basis for further questioning and exploration of the ELL, whether it is through biological experimentation of further computational experimentation. Biological experimentation can look for signs of the predictions made by the model's results. Further computational experimentation can test the model under different conditions than those tested in this thesis. The model can also be extended and refined to capture the ELL's function more accurately and test hypotheses that are not testable with the model in its current state.

\subsection{Limitations}

This thesis' model of the ELL is incomplete in many ways. It uses a reduced number of cells types than exist in real ELLs. The cerebellar-like structure which was adopted as a minimal relevant structure is even further reduced by removing the stellate cells. The cells that were incorporated were modeled approximately with 2D-IF neurons. The amount of inputs to the ELL was reduced, and the inputs that were incorporated were greatly simplified. The model only captures layers of cells along a single 2-dimensional plane and the sensory input to the model ELL consists of a one-dimensional array of values that represent stimulus intensity. Real ELLs are three-dimensional, and packed with cells that are connected across these dimensions. Their sensory input comes from 
the entire surface area of mormyrid's body, rather than a single line on one side of its body.

The mechanism of synaptic plasticity is simplified as well. In reality, there are many complex molecular interactions that occur in neuronal synapses for synaptic change to take place. These interactions produce complex dynamics. But in the ELL model, these dynamics are simplified into a single dimension that captures a general "weight" value that is multiplied by the synaptic connection's conductance to obtain a weighted conductance. The computation of weights happens only once per run, whereas real neurons are constantly changing.

The simplification and approximation of components in the ELL's structure, external connections, input patterns, and synaptic plasticity limit the model's ability to reproduce fully accurate and realistic activation patterns. These limitations arise from limited ability of a simple model to fully capture a detailed and complex system. But models are not all created in vain; by focusing the modeling effort on the main underlying components that drive the ELL's function, a simple model is able to capture general patterns that can describe the system.

There are other limitations to this thesis project other than modeling limitations. The model that was constructed was not able to reproduce the desired functions as completely as a real ELL would. Results demonstrate that the model was not able to completely capture the ELL's ability to remove sensory consequences of tail movement. There was a sign of some adaptation after a long training session, but the removal of the sensory consequences of tail movements was only partial. This incompleteness could 
potentially have occurred due to poor modeling of the input and STDP, or maybe more than two proprioceptive inputs are required to produce a full adaptive filter.

The approach of interpreting results used in this thesis was also limited. Visual comparison was used to evaluate visualizations of the simulation against graphs of real electrophysiological data. This visual technique provides but a preliminary approach for deriving results. The simulation data should be further scrutinized with statistical measures that can quantify differences between the model's simulated data and real ELL data. Such a quantitative comparison would be more exact, and might lead to further insight regarding the model's differences and how they might be fixed.

\subsection{Future work}

The limitations described in the previous section can all be addressed in future work: the model can be extended to additional dimensions, complex stimuli can be injected and the response studied, inputs can be added and refined, and plasticity can be more accurately modeled and implemented. Though there appears to be a lot of work to do, there are several particularly interesting and important things that can be studied by making only a few additions to the model. Of high importance is creating statistical measures that can quantify the comparison between the simulation data and real electrophysiological data. This would be a more exact approach to comparison than the visual approach used in the research reported in this thesis.

The deficits in efferent activity should be addressed before the model is extended much further. The timing of the efferent spikes and the number of cycles taken to produce the bursts in the LG cells following the removal of stimulus are far from 
accurate. As mentioned in the previous chapter, this is possibly due to the timing of the stimulus' response in MG cells. The effect of the timing of cellular responses should be examined, and should be fixed to reproduce the data described in Mohr et al (2003). This data demonstrates that the timing of electrosensory responses is later and more variable in MG2 cells than the other cells incorporated in this thesis' model. If MG2 cells responded later to electrosensory stimuli then that would likely improve the adaptive behavior of MG2 and LG cells.

Future work should also test the proprioceptive inputs to see what is required for the sensory consequences of movement to be predicted and removed from the sensory stream in a more complete way than demonstrated by the current model. The proprioceptive inputs implemented in this thesis were not sufficient to generate a complete adaptive filter that removes the sensory consequences of moment. This indicates that there is something missing from the model, either due to an incorrect theory, an incomplete theory, or defective implementation. Future work should examine these possibilities. Neurophysiological experimentation can attempt to localize the proprioceptive inputs and measure the effects of synaptic plasticity on them. The model should be extended to incorporate more proprioceptive than the two used for this thesis, and the implementation of STDP should be further scrutinized to see if it can be further refined to improve the adaptive filter.

If the effects of proprioceptive inputs are successful, then the model can be further used to demonstrate the data on the ELL's information filtering shown in Fig. 7.1. This figure, previously shown in Chapter 3, demonstrates that information content about objects when the tail is moved is different than when the tail is not moved before 
reaching the MG cells, and the information content is the same after passing the MG cells. This suggests that the MG cells make it so the effect of body position is negated. In this figure a measure of information called "SSI" is measured for afferent cells in Figure C and efferent cells in Figure D.

Producing such a graph was one of the initial goals of this project, and was discussed in Chapter 3: Formulation of Research Topic. However, the requirements to examine this did not develop adequately as other issues became more pertinent. To produce a graph that can be compared to Fig. 7.1 would require creating an informational unit that would capture the informational properties of afferent and efferent spike patterns. Two scenarios must be simulated, one with only objects as stimuli, and another with tail movements affecting the sensory stimulus of the object. Informational measures of afferent activity and efferent activity must be acquired in these scenarios, and can be graphed as in Fig. 7.1.
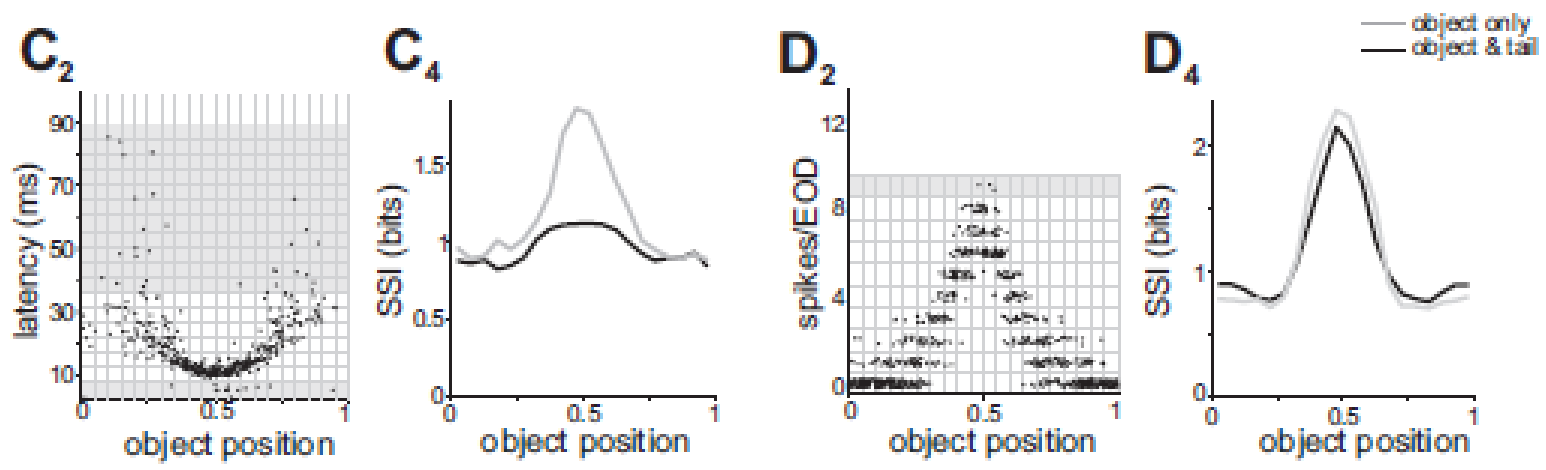

Fig. 7.1 Afferent and efferent informational measures (Sawtell \& Williams, 2008) 


\subsection{Conclusion}

The aim of this thesis was to bridge the gap between our scientific understanding of the ELL's structure and scientific understanding of its function by building a computational model of the ELL's relevant structure and testing to see if it reproduced the ELL's observed functional patterns. The systems science perspective was used to identify the relevant structure by breaking the system up into its structural components and relevant environment. The cerebellum-like structure within the ELL was chosen, along with inputs from electrosensory afferents, the electric organ corollary discharge, and proprioceptive inputs.

The approach of computational neuroscience was applied to model the system structure of the ELL and simulate its behavior. The cellular components were captured with 2-dimensional integrate and fire neurons, which can reproduce neuronal activity quite nicely and at a reduced computational cost. These neuronal models were interconnected according to the functional organization of the cerebellum-like structure observed in the ELL. These connections consist of excitatory and inhibitory connects, electrical synapses and chemical synapses. Inputs were simplified and injected as current into the ELL. Spike timing dependent plasticity was modeled and implemented at the MG cell's parallel fiber synapses. The effect of tail movement on sensory input was incorporated.

After the modeling was complete, its activity was simulated under multiple scenarios and visualized in ways that allowed us to scrutinize the details of the ELL's activity. The MG and efferent cells' activity in response to a stimulus' onset and offset was examined and compared to data acquired from real ELLs. Results from this 
comparison confirmed the sufficiency of the cerebellum-like structure in producing the general patterns that have been recorded in these cells. A point of contention was noticed in the efferent cell's response delay following stimulus removal. The reasons for this are unknown, and are not described by theory.

The adaptive filter's ability to remove the consequences of body movement was examined by simulated a pre-training, a training, and a post-training session that allowed for the comparison of the ELL's activity before training and after training. These results demonstrate that the proposed model is sufficient for producing a partial filter, which removes the consequences of body movement only to a limited degree. This was taken positively, as confirmation that the model can produce this effect. Further research should examine the model and the real ELL for the missing components that would be able to complete the filter.

Two hypotheses were tested. The first hypothesis tested if MG cell plasticity was sufficient to produce apparent plasticity observed in efferent cells. Results confirmed this hypothesis by showing plastic-like changes in efferent activity due to the plastic changes of MG cells. The second hypothesis tested the difference between gap junction and chemical synapses between granular cells and MG2, as well as between granular cells and LF cells. Results did not find any difference, and leave the hypothesis to be tested in future studies.

The computational model created for this thesis provides a skeleton for future studies to build on and improve. It acts as a preliminary bridge that can be used to bring the components of electroreception together into a unified model that can simulate the electrosensory function. This is an extremely effective method for hypothesis testing and 
validating a component's role in producing electroreception. By coordinating the development of the model with scientific efforts in the field of biology, data from the real system can be used to guide the model and the model can generate simulations that predict patterns of activity in the real system. These predicted patterns can be looked for in the real system, and the differences found can be scrutinized and theorized upon. Results drive further development and progress. 


\section{References}

Arnegard ME, Carlson BA. Electric organ discharge patterns during group hunting by a mormyrid fish. Proc Biol Sci. 2005 July 7; 272(1570): 1305-1314.

Beer R.D. and Chiel H.J. Computational neuroethology. Scholarpedia. 2008.

Bell CC. Memory-based expectations in electrosensory systems, Curr Opin Neurobiol. 2001Aug; 11(4):481-7.

Bell CC. Mormyromast electroreceptor organs and their afferent fibers in mormyrid fish. II. Intra-axonal recordings show initial stages of central processing. J. NeurophysioI. 1990; 63:303-318.

Bell CC. Sensory coding and corollary discharge effects in mormyrid electric fish. J Exp Biol. 1989 Sep; 146:229-53.

Bell CC, Han VZ, Sugawara Y, Grant K. Synaptic plasticity in a cerebellum-like structure depends on temporal order. Nature. 1997 May 15; 387(6630):278-81.

Bell CC, Han VZ, Sugawara Y, Grant K. Synaptic plasticity in the mormyrid electrosensory lobe. J Exp Biol. 1999 May; 202(pt 10):1339-47.

Bell CC, Han VZ, Sawtell NB. Cerebellum-Like Structures and Their Implications for CerebellarFunction. Neuroscience. 2008 Jul; Vol. 31: 1-24.

Bullock TH, Heiligenberg WF. in: Bullock TH, Hopkins CD, Popper AN, Fay RR. Electroreception. New York, John Wiley \& Sons. 1986; pp 527-576.

Bullock TH, Hopkins CD, Popper AN, Fay RR. Electroreception. New York: Springer. 2005.

Engelmann J, Bacelo J, Metzen M, Pusch R, Bouton B, Migliaro A, Caputi A, Budelli R, Grant K, von der Emde G. Electric imaging through active electrolocation: implication for the analysis of complex scenes. Biol Cybern. 2008 Jun; 98(6):51939.

Han VZ, Bell CC, Grant K, Sugawara Y. Mormyrid electrosensory love in vitro: morphology of cells and circuits. J. Neurophysiol. 1999; 404:359-374. 
Han VZ, Grant K, Bell CC. Rapid activation of GABAergic interneurons and possible calcium independent GABA release in the mormyrid electrosensory lobe. J. Neurophysiol. 2000; 83:1592-1604.

Hodgkin AL and Huxley AF. A Qualitative Description of Membran Current and its Application to Conduction and Excitation in Nerve. J. Physiology. 1952; 117 : 500-544.

Hopkins CD. Stimulus filtering and electroreception: Tuberous electroreceptors in three species of Gymnotoid fish. J Comp Physiol A Neuroethol Sense Neural Behav Physiol. 1976; Vol. 111\#2, 171-207.

Izhikivich EM. Dynamical Systems in Neuroscience. MIT Press: Cambridge. 2006.

Kleinfeld D, Ahissar E, Diamond ME. Active sensation: insights from the rodent vibrissa sensorimotor system. Current Opinion in Neurobiology. 2006 Aug; 435-444.

Lendaris, GG. On Systemness and the Problem Solver. IEEE Transactions on Systems Man and Cybernetics. 1986 July/August; 604-610.

Meek J, Grant K, Bell CC. Structural Organization of the Mormyrid Electrosensory Lateral Line Lobe. The Journal of Experimental Biology. 1999 April; 1291-1300.

Mohr C, Roberts PD, and Bell CC. The Mormyromast Region of the Mormyrid Electrosensory Lobe. I. Responses to Corollary Discharge and Electrosensory Stimuli. J Neurophysiol. 2003; 90:1193-1210.

Roberts PD. Electrosensory response mechanisms in mormyrid electric fish, Neurocomputing. 2000; 32-33:243-248.

Roberts PD and Hayes T. Lecture material from Computational Neuroscience course: http://www.bme.ogi.edu/BME665/. 2007.

Roberts PD, Leen TK, Sawtell NB, Case S, Hunt J. Processing of spatial electrosensory patterns in mormyrid electric fish: Tools for investigations. CRCNS Poster. 2010.

Roberts PD, Portfors CV. Design principles of sensory processing in cerebellum-like structures. Biological Cybernetics. 2008; 491-507.

Sawtell NB. Multimodal integration in granule cells as a basis for associative plasticity and sensory prediction in a cerebellum-like circuit. Neuron. 2010 May 27;66(4):573-84.

Sawtell NB, Williams A, Bell CC. Central control of dendritic spikes shapes 
the responses of Purkinje-like cells through spike timing-dependent synaptic plasticity. J Neurosci. 2007 Feb; 14;27(7):1552-65.

Sawtell NB, Williams A. Transformations of electro sensory encoding associated with an adaptive filter. J Neurosci. 2008 Feb 13;28(7):1598-612.

Spencer JP, Perone S \& Johnson JS. Dynamic Field Theory and Embodied Dynamics. In Spencer JP, Thomas MS, \& McClelland JL (Eds.)Toward a Unified Theory of Development: Connectionism and Dynamic Systems Theory Re-Considered. New York: Oxford University Press. 2008.

Sporns O. Networks of the Brain. The MIT Press: Cambridge. 2010.

von der Emde G. Active Electrolocation of Objects in Weakly Electric Fish. The Journal of Experimental Biology. 1999; 202, 1205-1215.

von der Emde G. Non-visual environmental imaging and object detection through active electrolocation in weakly electric fish. J Comp Physiol A Neuroethol Sense Neural Behav Physiol. 2006 Jun; 192(6):601-12.

Wallish P, Lusignan M, Benayoun M, Baker TI, Dickey AS, Hatsopoulos NG. MATLAB for Neuroscientists. Academic Press. 2009.

Zakon HH, Thomas P, Yan HY. Electric organ discharge frequency and plasma sex steroid levels during gonadal recrudescence in a natural population of weakly electric fish. J Comp Physiol A Neuroethol Sense Neural Behav Physiol. 1991; 169: 493-499.

Zupanc KHG, Bullock TH. From Electrogenesis to Electroreception: an Overview. In: Bullock TH, Hopkins CD, Popper AN, Fay RR Electroreception. New York: Springer. 2005. 


\section{Appendix: MG and Efferent layer MATLAB program}

This appendix contains the MATLAB program for the main MG and efferent cellular layer used in this thesis. This layer receives input from a separate function for granular cell activity, and it uses separate functions such as EOCD to determine parallel fiber inputs, and STDP to determine synaptic weight change for each run. This MG and efferent program is called upon by a different program through which the stimulus is controlled as well as other important experimental parameters.

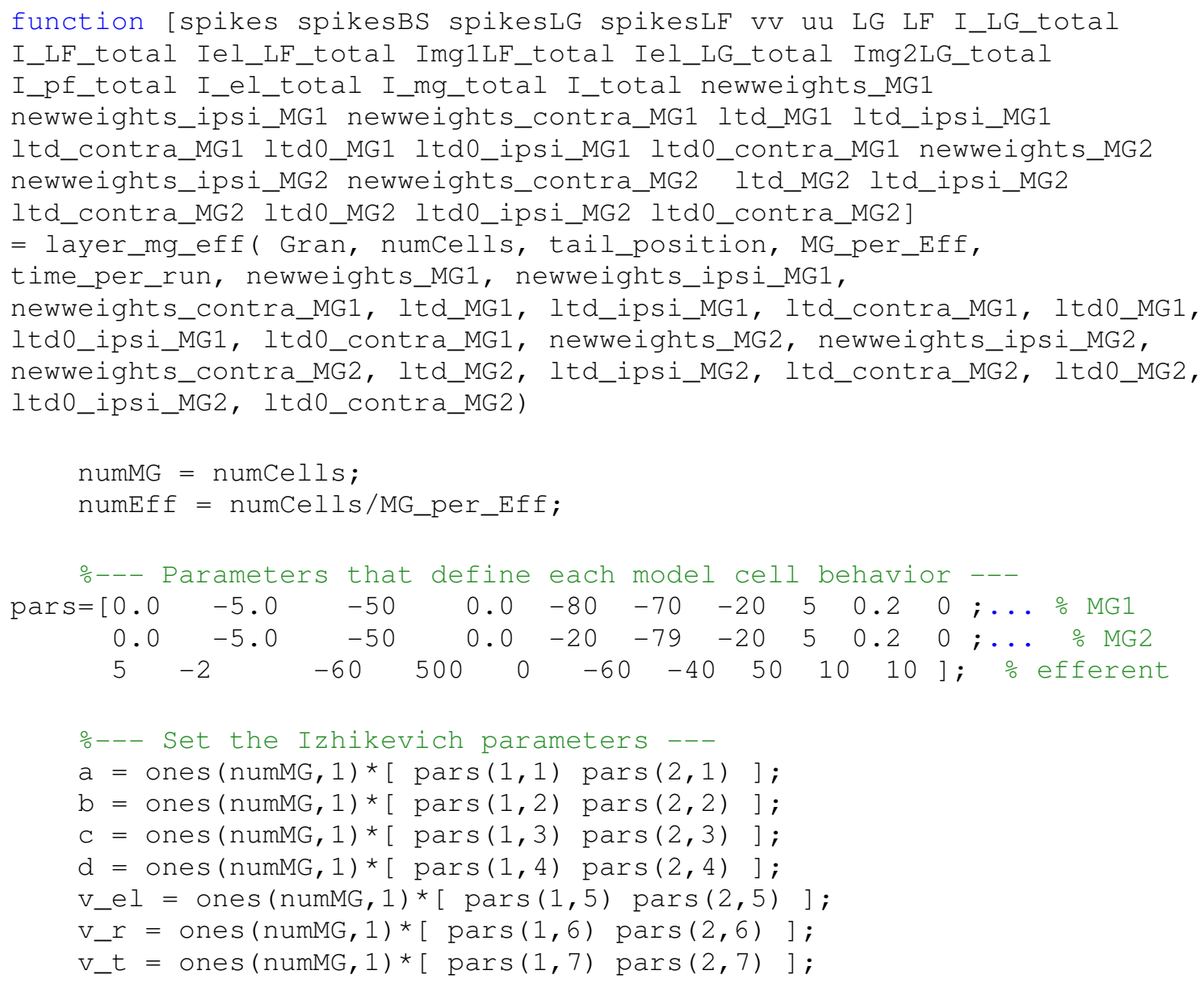




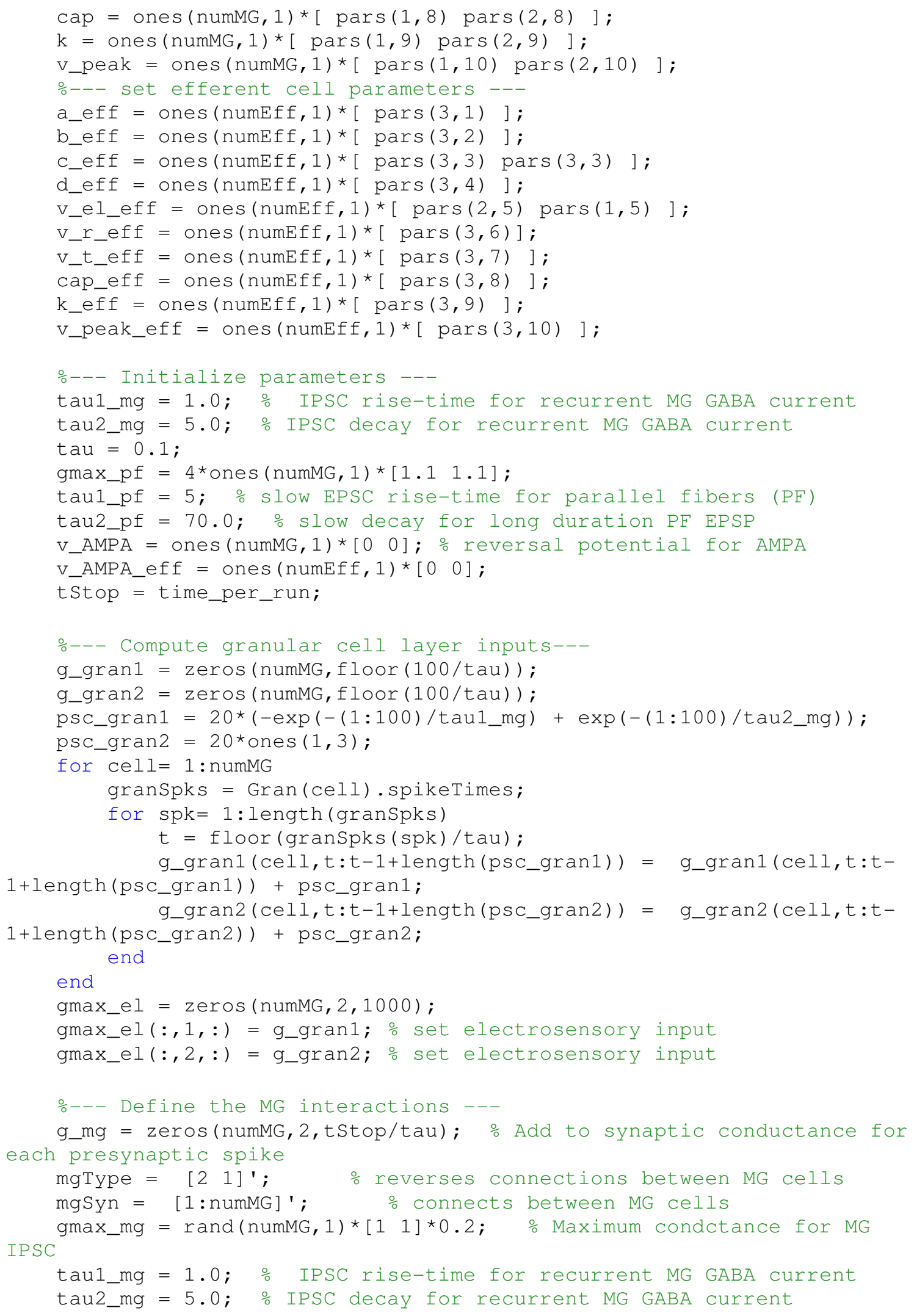




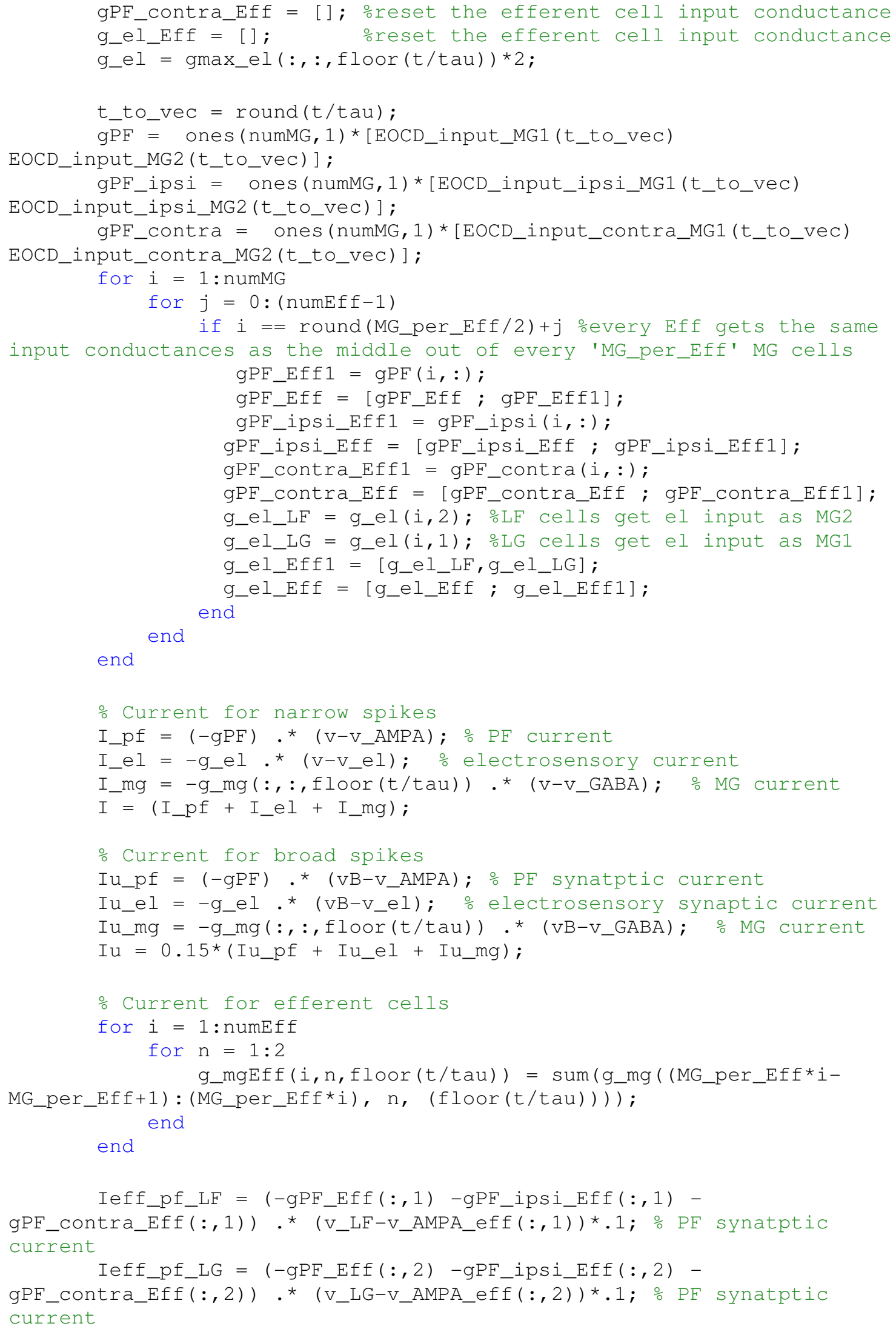




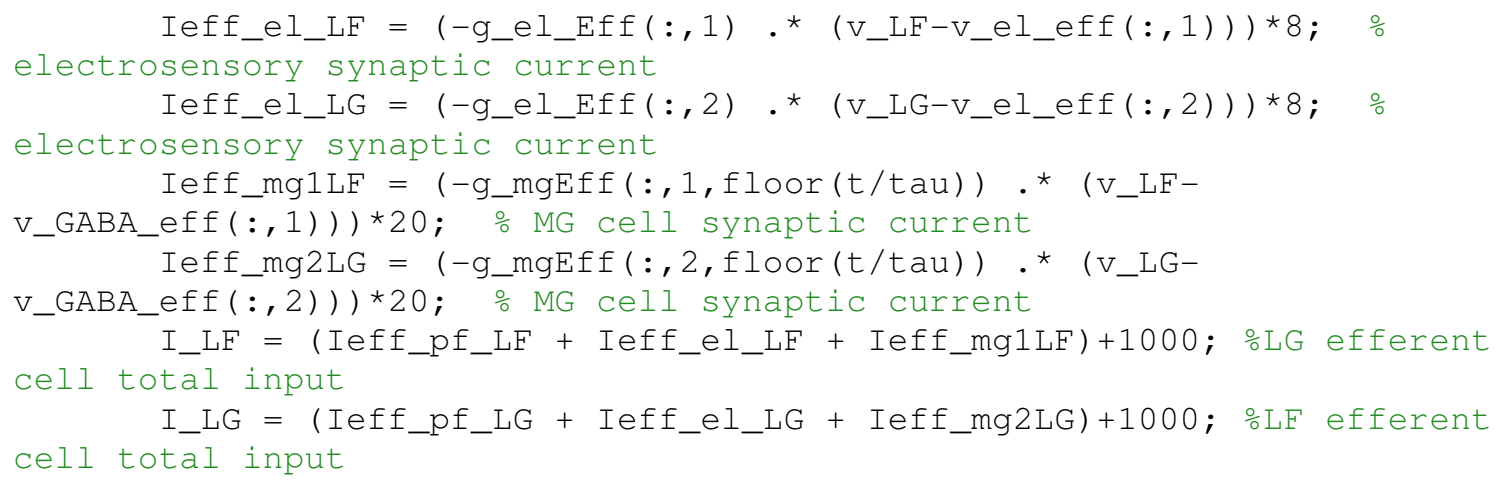




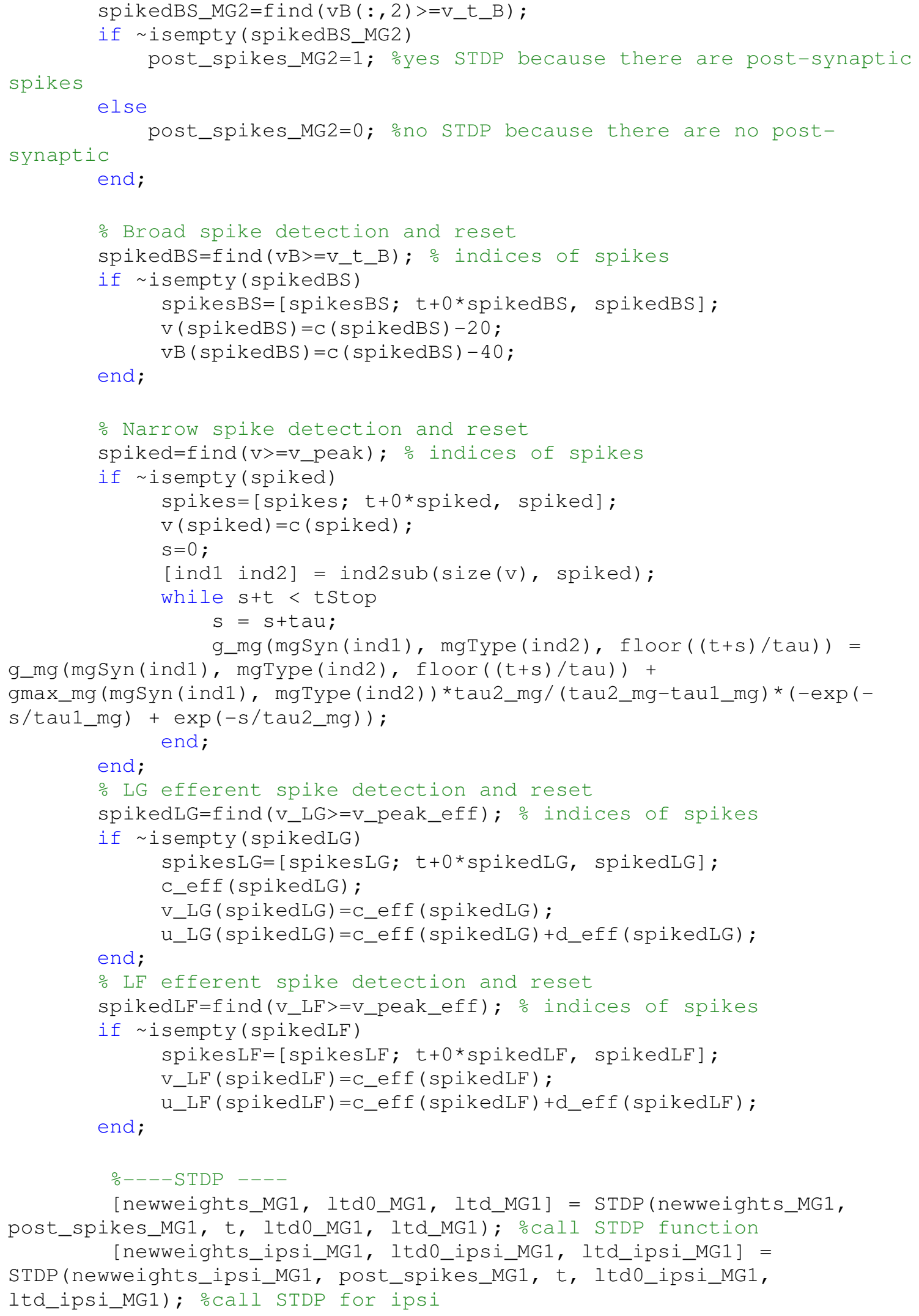


[newweights_contra_MG1, ltd0_contra_MG1, ltd_contra_MG1] = STDP (newweights_contra_MG1, post_spikes_MG1, t, 1tdo_contra_MG1, ltd_contra_MG1); \%call STDP for contra

[newweights_MG2, 1td0_MG2, 1td_MG2] = STDP(newweights_MG2, post_spikes_MG2, t, ltd0_MG2, ltd_MG2); \%call STDP function

[newweights_ipsi_MG2, ltd0_ipsi_MG2, ltd_ipsi_MG2] =

STDP (newweights_ipsi_MG2, post_spikes_MG2, t, ltd0_ipsi_MG2, ltd_ipsi_MG2); \%call STDP for ipsi

[newweights_contra_MG2, ltd0_contra_MG2, ltd_contra_MG2] = STDP (newweights_contra_MG2, post_spikes_MG2, t, ltd0_contra_MG2, ltd_contra_MG2); \%call STDP for contra

end; 